PREPOSITIONING OF EMERGENCY SUPPLIES FOR DISASTER RESPONSE

\author{
A Dissertation \\ Presented to the Faculty of the Graduate School \\ of Cornell University \\ In Partial Fulfillment of the Requirements for the Degree of \\ Doctor of Philosophy
}

by

Carmen Gloria Rawls

August 2008 
(C) 2008 Carmen Gloria Rawls 


\title{
PREPOSITIONING OF EMERGENCY SUPPLIES FOR DISASTER RESPONSE
}

\author{
Carmen Gloria Rawls, Ph. D.
}

Cornell University 2008

Extreme events such as hurricanes and earthquakes can strike a community with little or no warning and leave high levels of devastation behind. Emergency response providers require large quantities of resource in the aftermath of such events, but these may be limited because of lack of preparation. In order to provide immediate assistance to disaster victims, essential supplies must be strategically placed before the event so they can be accessible after. The main goal of this research is to develop a large-scale emergency response planning tool that determines the location and quantities of emergency supplies together with the location and capacities of their storing facilities. A two-stage stochastic mixed integer program (SMIP) is presented that designs such an emergency response pre-positioning strategy for hurricanes or other natural disaster threats. The SMIP is a robust model that considers variability in forecasted demand and network unreliability.

Due to the computational complexity of the model formulation, a heuristic solution that considers the embedded network structures of the SMIP was devised by combining two methodologies: the L-shaped method and the Lagrangian relaxation. The L-shaped method consists of solving an approximation of a stochastic program by estimating the recourse function using an outer-linearization technique. The Lagrangian relaxation heuristic was added to decompose the first stage problem into a trivial facility location problem and a resource allocation linear program. To further improve the computational capabilities of the algorithm, the Lagrangian relaxation 
was also used to relax the integrality constraints of the facility location variables. The result was a heuristic method referred to as the Lagrangian L-shaped method (LLSM).

Various numerical experiments were conducted to test the computational capabilities of the LLSM. These experiments showed the computational consistency of the method compared to a standard integer program solver (i.e. Lingo). Regardless of variations in the data set provided, the running times of the LLSM are $0.05 \%$ to $10.0 \%$ of the Lingo running times, while the objective values obtained by the LLSM are within $1 \%$ of optimum. Based on the experiments, we are confident that the LLSM can be used as a large-scale resource pre-positioning planning tool. 


\section{BIOGRAPHICAL SKETCH}

Carmen G. Rawls received her Bachelor of Science Degree in Civil Engineering from the University of Puerto Rico in Mayagüez, Puerto Rico, in the year

2000. She received her Master of Science degree in Civil Engineering from the University of Illinois at Urbana-Champaign, Illinois, in 2003 to then pursue her Ph. D. at Cornell University in the field of Transportation Systems Engineering. 
To my loving family for all their support: my husband (Wilkins), my son (Robert Edward), and my parents (Robert and Pura). You are my strength and inspiration. 


\section{ACKNOWLEDGMENTS}

I would like to thank all my friends without whom this program would not have reached optimality.

I would like to thank my advisor, Mark Turnquist, for teaching me far more than transportation systems. He has shown me patience and care, values I hope to practice in my future research and life. I would like to thank my Special Committee members Linda Nozick and Arnim Meyburg, for their willingness to guide me through this endeavor.

Thanks to my parents for instilling in me the will and determination to reach my goals, no matter how high. I would especially like to thank my husband, the key term in this equation, for helping me every step of the way and for always believing in

me. To my son, thank you for forcing me to take breaks and giving new meaning to everything.

Finally, thanks to my friends who helped me bring balance to the force: Yashoda, Kabeh, Ali, Pantea, Adam, Verónica, Chi, and Yao. 
TABLE OF CONTENTS

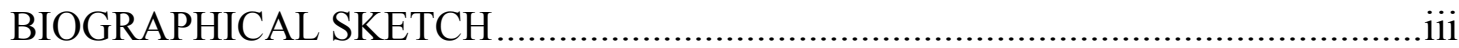

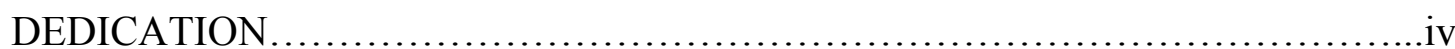

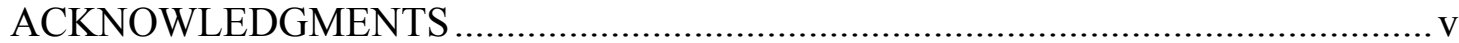

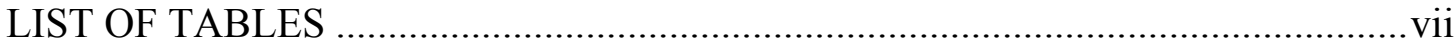

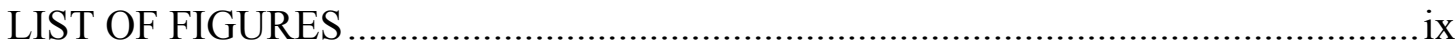

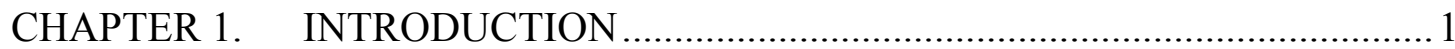

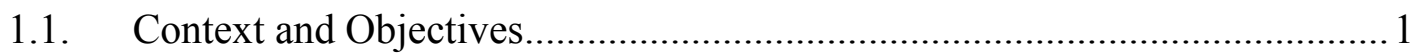

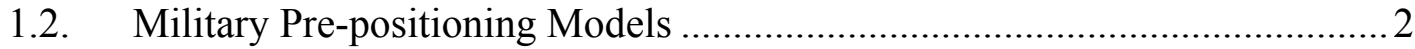

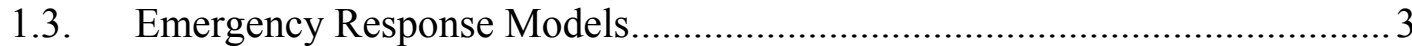

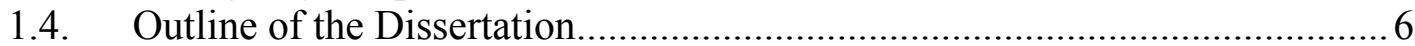

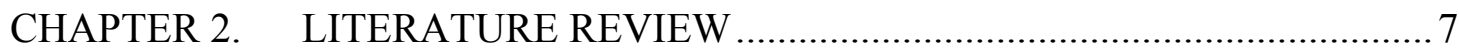

2.1. Facility Location Models.....................................................................

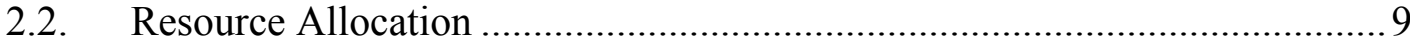

CHAPTER 3. STOCHASTIC MIXED INTEGER PROGRAM ………………..... 12

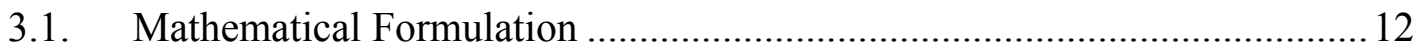

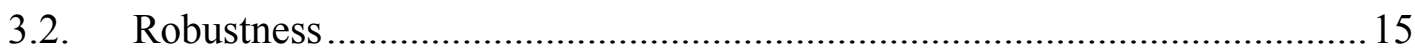

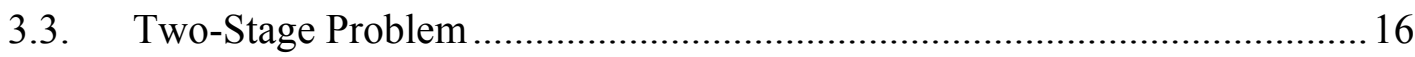

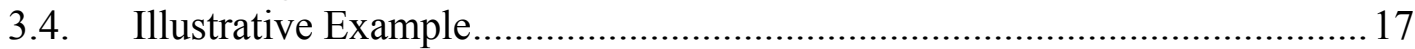

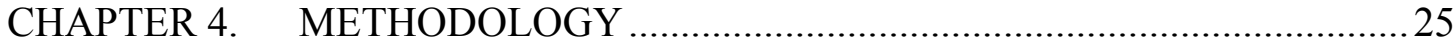

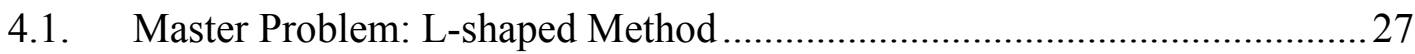

4.2. Sub-problem: Minimum Cost Flow Problem .................................................. 34

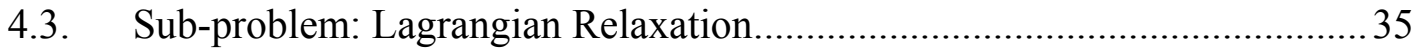

4.4. Lagrangian L-shaped Method Algorithm.................................................. 40

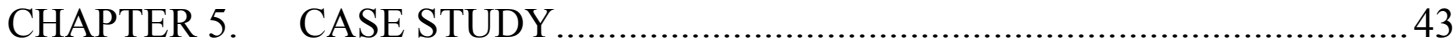

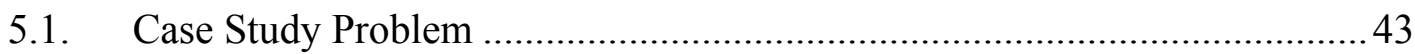

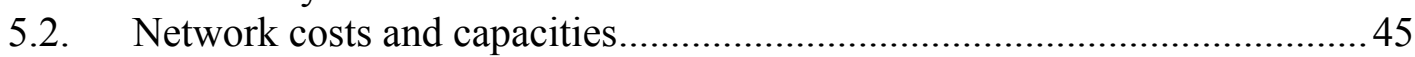

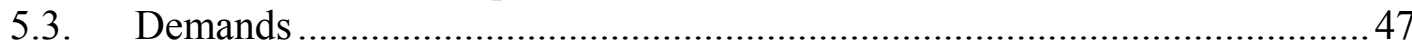

5.4. Resource and facilities unit costs and dimensions .....................................52

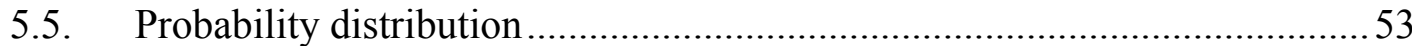

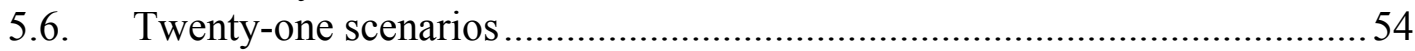

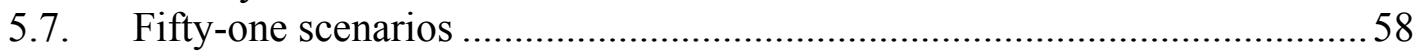

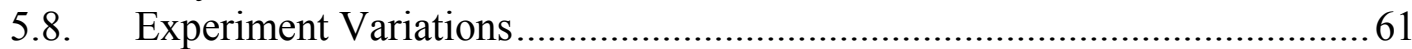

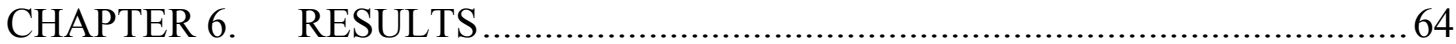

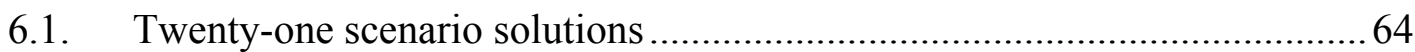

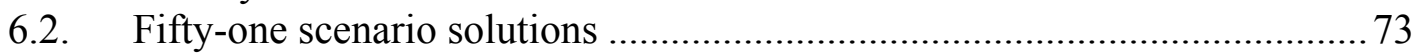

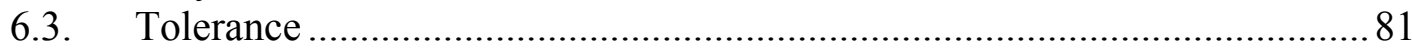

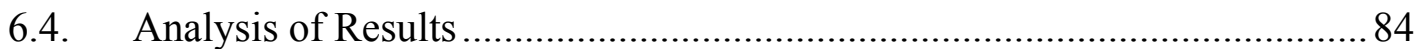

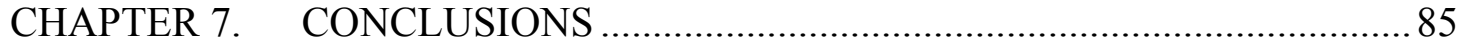

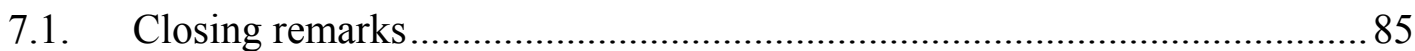

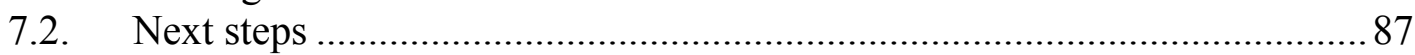

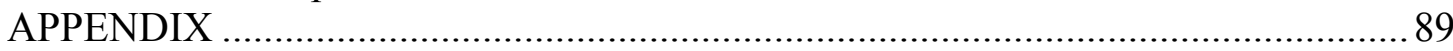

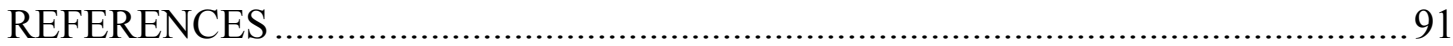




\section{LIST OF TABLES}

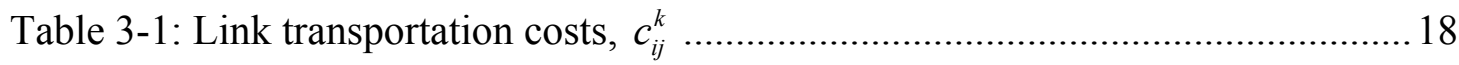

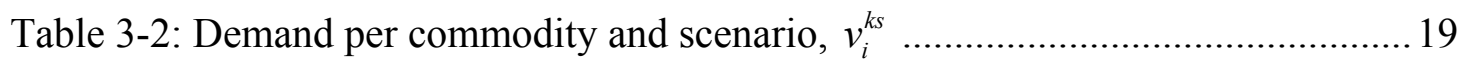

Table 3-3: Default link capacity for each commodity $(s=1), u_{i j}^{k s} \ldots \ldots \ldots \ldots \ldots \ldots \ldots \ldots \ldots . . . . . . . . . . . . . . .19$

Table 3-4: Fixed costs incurred with the opening of a warehouse per size and capacities associated with each warehouse size. ............................................. 19

Table 3-5: Purchase cost and volume space occupied per unit of commodity.............. 19

Table 3-6: Facility location and size with its respected allocated resource .................20

Table 4-1: Values per iteration of the first and second stage problems ....................... 32

Table 5-1: Nodes included in the network ............................................................. 44

Table 5-2: Roadway and facility damages resulting from hurricane passage ............. 46

Table 5-3: Emergency supply demands generated by hurricane threat........................49

Table 5-4: Demand generated by the hurricanes used to construct the scenarios by

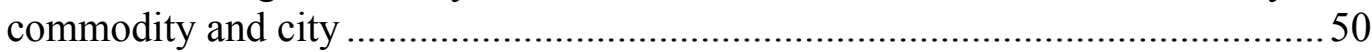

Table 5-5: Costs of opening a facility depending on its storage capacity .....................52

Table 5-6: Unit purchase price, transportation costs and volume occupied by

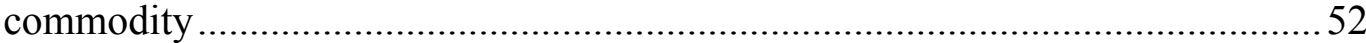

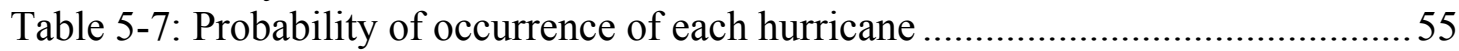

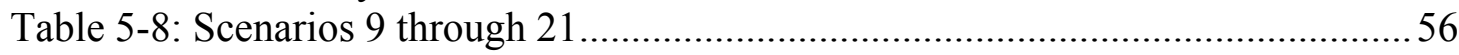

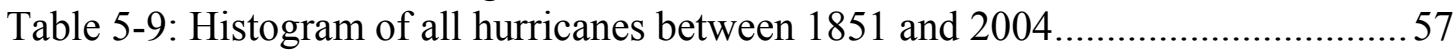

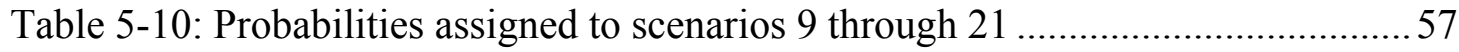

Table 5-11: Probability of occurrence of each of the 15 hurricanes ..........................59

Table 5-12: Probability computations for the hurricane paired scenarios...................60

Table 6-1: Results for 21-scenarios provided by the LLSM and Lingo .....................65

Table 6-2: First stage solutions obtained with the Lagrangian L-shaped method........66

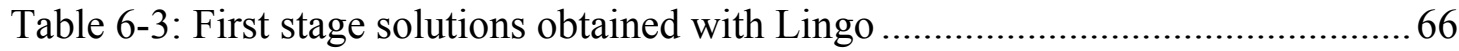

Table 6-4: Maximum unmet demand level per experiment of the 21-scenario case study .67

Table 6-5: Objective value composition for the LLSM and the Lingo solutions......... 68

Table 6-6: Objective values per stage per experiment of the 21-scenario case study .. 73

Table 6-7: Results for 51-scenarios provided by the LLSM and Lingo..................... 74

Table 6-8: First stage solutions obtained with the Lagrangian L-shaped method........ 75

Table 6-9: First stage solutions obtained with Lingo ........................................... 75

Table 6-10: Maximum unmet demand level per experiment of the 51-scenarios case

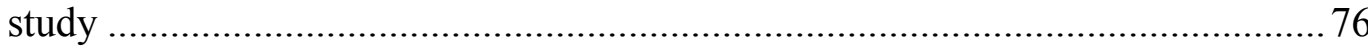

Table 6-11: Solution for case 1 (no planes, no bottleneck) ....................................... 77

Table 6-12: Solution for case 2 (planes and bottleneck) ........................................ 78

Table 6-13: Solution for case 3 (bottleneck) ....................................................... 78

Table 6-14: Solution for case 4 (planes) ............................................................ 79

Table 6-15: Results for 51-scenarios with variations in demand for hurricane Katrina in New Orleans and unmet demand penalty costs obtained with LLSM and Lingo 
Table 6-16: Solution errors related to the tolerance on second-stage approximation .. 82

Table 6-17: Tolerance error of the Lagrangian Relaxation ....................................... 83 


\section{LIST OF FIGURES}

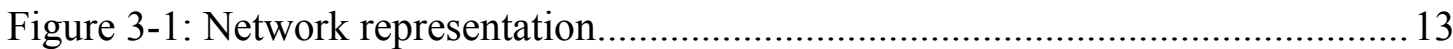

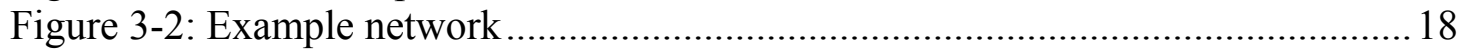

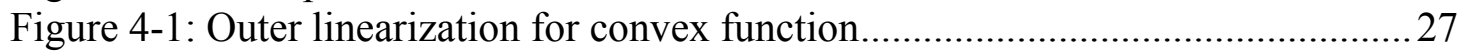

Figure 4-2: Second stage function and optimality cuts ................................................. 32

Figure 4-3: Greedy algorithm to determine the facility location and capacity...............38

Figure 4-4: Lagrangian L-shaped method algorithm................................................... 41

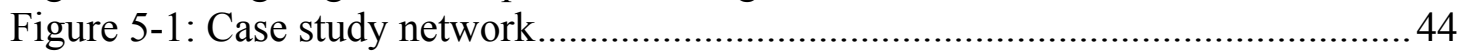

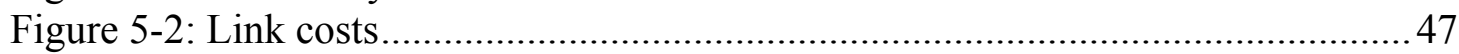

Figure 5-3: Commodities considered - water tanks, meals-ready-to-eat (MREs) and

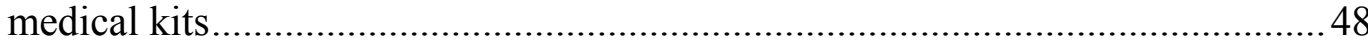

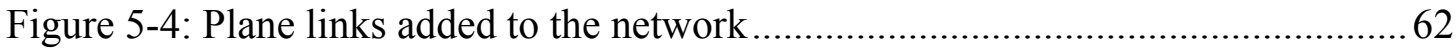

Figure 6-1: LLSM pre-positioning strategy case 1 (no planes, no bottleneck) .............69

Figure 6-2: LLSM pre-positioning strategy case 2 (planes and bottleneck) .................70

Figure 6-3: LLSM pre-positioning strategy for case 3 (bottleneck).............................. 71

Figure 6-4: LLSM pre-positioning strategy for case 4 (planes) ..................................... 72

Figure 6-5: Graphical representation of the effect of tolerance...................................... 82

Figure 6-6: Graphical representation of the effect of tolerance in the Lagrangian relaxation 


\section{CHAPTER 1. INTRODUCTION}

\subsection{Context and Objectives}

Extreme events such as tornadoes, earthquakes, hurricanes, or terrorist attacks strike a community with little or no warning and can leave high levels of devastation behind. Large quantities of supplies are needed in the aftermath of such events, but these may be limited due to lack of adequate preparation. Emergency response efforts become ineffective when supplies are unavailable or insufficient. Supplies must then be acquired from other regions incurring high shipment costs and taking too long to reach the victims. Damages in the network can result from such disasters; roads and facilities may be obstructed, destroyed and unavailable.

The main goal of emergency response is to provide assistance to disaster victims as soon as disasters strike, minimizing the number of casualties due to secondary effects such as aftershocks, building collapse or lack of proper medical assistance. In order to achieve this, essential supplies must be in place at strategic locations before the event so that they may be available immediately after. For similar reasons, risk mitigation and decrease in the response time, pre-positioning strategies are already in use by the military armed forces. These strategies permit a rapid and effective response to conflicts anywhere in the world. However, limited planning tools resulting from formal modeling techniques (optimization or simulation) have been developed, and the existent models fail to account for system unreliability.

The motivation behind this project arose from the need for facility location and resource allocation models that provide emergency response organizations with supply mitigation strategies prior to devastating events and may provide the military with robust equipment preposition strategies. The objective of the model proposed is to 
provide an emergency planning tool that determines the most accessible emergency supply locations and optimal quantities of resource that need to be acquired based on uncertain demand and unreliable network information. The unreliability of the network will include situations where the supply distribution facilities might be destroyed and connecting paths might be obstructed during a catastrophic event. The model is formulated as a mixed integer stochastic program.

The remainder of this chapter contains the following. Sections 1.2 and 1.3 include a general discussion of research on military pre-positioning models and emergency response, setting the stage for the model description. Section 1.4 presents the outline for the remainder of the dissertation.

\subsection{Military Pre-positioning Models}

The concept of pre-positioning of key resources is deeply embedded in military planning operations. This strategy permits speed of response, flexibility and safety against emerging threats regardless of geographical limitations. It has been stated by the Overseas Basing Commission (2005) that their operational capability depends on the location of their pre-positioned unit sets, ammunition stocks and other supporting items, what they are comprised of, how they are maintained, defended, and continually updated. Furthermore, their speed of entry, appropriate force packaging, flexibility and levels of combat power rely on having the right equipment and supplies in place.

Pre-positioning strategies are used to determine the location of a wide range of military elements, from tents, food, kitchens, shelters, power equipment, to the location of actual military bases. Since they measure the success of pre-positioning by the degree of combat readiness, they include the element of reliability (e.g. reliable non-corroded and fully functional vehicles and equipment, Le Pera (2004)). The 
success in applying pre-positioning strategies can be seen in the ability of Air Force Engineering and Services during Operation Desert Shield to move within days enough supplies to support 21,000 personnel (Lally, 1991). However, regardless of its importance to the armed forces, few modeling tools have been developed to tackle this problem. A recent effort is a model created by Johnstone, et al. (2004) for prepositioning and movement of munitions for the U. S. Air Force. This model was formulated as a mixed integer program and creates optimal plans for a given set of demands in a specific scenario. The model does not consider transport vulnerability, demand or network uncertainty.

The Overseas Basing Commission (2005) expressed their need for a tool that determined the amounts and composition of the supplies and equipment to be prepositioned considering their budget, uncertainty of access to the supplies, and investment costs. They also indicated how pre-positioning planning should be performed keeping in mind flexibility and alternatives due to the constantly shifting geopolitical landscape.

\subsection{Emergency Response Models}

Previous research regarding emergency response topics have concentrated mostly on disaster management following natural disasters, terrorist attacks and hazardous materials accidents. Some of the concerns expressed have been prompt response and decision making strategies under crisis conditions. Their main goal has been to develop emergency response plans that integrate information pertaining to the location and capacities of resource providers, the spatial distribution of the victims, the

environment and the economy (Parentela et al., 2000). Even though the location and capacities of the resource providers are key components in the disaster management 
plan, little research has been conducted on the topic of a priori planning - i.e. what resources should be stockpiled in what location so that emergency response is most effective in the event that it is needed.

Several geographical information systems (GIS) applications have been developed to assess the damage caused by a disaster, identify safe routes, and for resource mapping. The model developed by Al-qurashi (2004) combined GIS with an emergency response system (ERS) with the purpose of providing emergency responders such as firefighters or medical personnel with information on the status of the network. In this case, the event studied is a gas leak and GIS is used to show the gas contamination dispersion. Parentela et al. (2000) developed GIS applications for immediate response to emergency situations by redirecting response units based on their real time locations. Tsai et. al. (2002) developed a prototype Information Technology-based Real-time Emergency Response system framework that dispatches emergency vehicles to demand points based on their real-time location acquired with GIS/GPS techniques. An illustration of an a priori resource location approach is the work by Sathe and Miller-Hooks (2005), a robust mixed integer linear program that assigns and re-assigns the locations of first response units (e.g. military units, police forces) in order to maintain protection coverage to critical facilities considering changes in the state of the system. An attempt at determining the location of facilities that distribute medical supplies and assistance (e.g. pharmaceutical caches and staging areas) for a large scale emergency (e.g. anthrax virus in Los Angeles County) was tackled by Jia et al. (2007). They solved a set covering problem formulation with predetermined demand via various heuristic methods. An a priori facility location model was proposed by Saccomanno and Allen (1988) that determines, using a minimum coverage algorithm, the location of response-capable centroids (i.e. fire stations or 
police stations) that could provide aid in case of spills of dangerous goods on a rural road network.

Utility service providers play a critical role in the system recovery process. Since they need to resume service in the least amount of time possible they have devised strategies that take place before and after a disaster strikes. Power plants for example, prepare for the disaster in the planning stage by designing a more reliable network. Research for reliability planning has developed techniques for determining capacity and location of capacitor banks (Makram et al., 1995; Chin, 1995; Ng and Salama, 1995) and other electric utility distribution elements (El-Khattam et al. 2005). After the disaster, these companies optimize their maintenance force in order to minimize the recovery period in the operational stage. An algorithm designed for the operational stage is presented by Guha et al. (1999) in which they try to reconnect the customers to the network in the least amount of time possible by optimal workforce assignments. Their model is a budgeted maximum coverage problem that provides assistance based on customer importance. Priorities are given to hospitals or any other emergency service provider. Similarly, a mathematical model created by Weintraub et al. (1999) dispatches emergency vehicles in order to provide service in the least amount of time possible. Priorities are given to areas with higher number of expected failures.

There is a need for a robust emergency response planning tool to determine the location and the capacity of the facilities where essential resources should be stored, and the quantities and types of those resources to be pre-positioned in each location. The model should consider both network and demand uncertainty. A stochastic mixed integer program is proposed and presented in Chapter 3 that meets these requirements by considering uncertain demand, disastrous events striking different areas in the network, the fixed costs associated with the addition of storage facilities of different 
capacities, the resource purchase costs, shipping costs and/or travel times, and uncertain network capacity. This model provides a flexibility lacking in previous efforts.

\subsection{Outline of the Dissertation}

Chapter 2 includes a summary of related work in facility location and resource allocation problems. Chapter 3 contains the formulation of the mathematical Stochastic Mixed Integer Program (SMIP). Chapter 4 describes how the SMIP is decomposed into three less computationally expensive sub-problems based on its embedded structures (i.e. facility location, resource allocation and network flow problems). Chapter 4 includes the methodology used to solve the SMIP, an algorithm that combines Network Simplex, the L-Shaped Method and Lagrangian Relaxation approaches. In Chapter 5 an illustrative case study is presented. Chapter 6 includes the results of the experiments conducted. Chapter 7 provides the conclusions and the directions for continuing work. 


\section{CHAPTER 2. LITERATURE REVIEW}

The model presented in Chapter 3 is a stochastic mixed integer program (SMIP) representing both capacitated facility location and resource allocation. The goal of this research is to determine the optimal number, location and capacity of emergency response facilities providing supplies and service to regions potentially affected by a natural disaster. The model also assigns to each facility an amount of each of several resources that would minimize the average transportation cost while maximizing the demand met. The model has elements found in emergency response, facility location and resource allocation problems. Relevant research in these areas is discussed briefly.

\subsection{Facility Location Models}

Facility location models are important topics in operations management and operations research since the location of distribution systems determine the quality of service. These models can determine the best location based on lowest operational costs, market competition or demand concentrations. The proposed model has embedded properties of fixed costs or fixed charge facility location (FCFL) models as defined by Bradley et al. (1977). These models select the location of the facilities and assign the customers (or demand) to the facilities minimizing fixed and transportation costs. A difference between the SMIP and the FCFL models is that for the second, the demand must be met while in the SMIP model unmet demand is penalized but not forbidden. Another difference between the models is that in the FCFL models there are typically no arc capacities and often no facility capacities. 
The SMIP model can also be compared to p-median facility location models. They are similar in that both are concerned with total transportation costs for moving materials to the demand points. They are different in that no fixed number of facilities is imposed on the SMIP. Research conducted on related p-median topics include models developed by Berman et al. (2003) and Syam (1997). These authors modified p-median problems in order to incorporate reliability and facility capacity. Berman et al. (2003) added reliability of service with a nonlinear programming model that maximized the expected demand met with satisfactory service. Reliability and thus, the quality of service were measured by the distance traveled by the supply vehicles. Berman et al. (2003) did not limit the possible locations for the facilities but restricted their number. Their model is similar to the p-median problem in that it limits the number of facilities but contrary to the p-median problem the demand points do not necessarily receive service from the closest service facility. On the other hand, Syam (1997) extended the p-median problem by not only restricting the number of open facilities in a region but also their available capacity. The author also investigated the effects of additional managerial restrictions while always meeting the demand.

Past efforts in emergency response planning have solved facility locations as set covering problems. Their goal has been to minimize the number of open facilities without sacrificing service quality. For example, a pre-set maximum permitted distance (cost or time) traveled by emergency vehicles can be specified (Toregas et al., 1970). Neebe (1988) avoided finding the maximum limit a priori by developing a heuristic model that found the tradeoffs between distance and number of facilities needed to meet the demand. The SMIP lets the model determine this distance by incurring high penalty costs for unmet demand. Therefore, the number of facilities and how far they would be located from the demand points will be a decision of the model. 
To include the effects of a natural disaster or a terrorist attack in the model, network reliability will be included in the scenarios set. Similar to the model by Snyder and Daskin (2003) the proposed model quantifies the effects of lack of reliability with additional transportation costs, but also with unmet demand penalty costs. Few facility location models have included facility performance reliability during its operation. The model by Snyder and Daskin (2005) minimized fixed costs for opening the facilities and also the expected transportation costs incurred due to facility failures. In the model presented by Hsieh and Chen (2005a, 2005b) network reliability was established with probability density functions assigned to the nodes and arcs in the network.

\section{2. $\quad$ Resource Allocation}

Hsieh and Chen (2005a, 2005b) developed resource allocation models for unreliable networks, using multi-source multi-sink flow networks that minimize the quantities of resources required at the source nodes in order to satisfy demand. Their objective was to maximize the reliability that the resources reach their destination. The model was solved using a modified enumerative method. In their models the arcs and the intermediate nodes in the network are unreliable and are modeled as statistically independent random variables. Contrary to the SMIP, their models restrict the overall transmission costs incurred, force all the resources located at the facilities to be shipped and the demand to be met. In addition, not only the intermediate nodes but all the nodes in the network are unreliable in the proposed model.

Various models have been created for resource scheduling as risk management applications. Fiedrich et al. (2000) provided a dynamic optimization model that minimizes the number of expected fatalities resulting from an earthquake by assigning 
search and rescue resources for the initial search-and-rescue period. The model seeks an optimal schedule that assigns emergency response resources in space and time to the areas affected by an earthquake considering the time limit, the quality and quantity of the resources. Their main concern was to decrease the number of fatalities that occur by secondary damages, delayed rescue, and lack of medical assistance. The resources that comprise the schedule include search and rescue teams, transportation of victims to hospitals, reconstruction and rehabilitation of certain facilities and path segments.

A second attempt was presented by Sherali et al. (2004). Their model is a nonconvex emergency response resource allocation model solved as a tight linear programming relaxation with an embedded branch and bound framework. Their emergency response model allocates available resources based on system efficiency and equity considerations. The goal is to provide emergency managers with a tool that deploys the available resources so as to minimize the weighted mitigated risk in the system. Note that information on the level of damage caused by the disastrous event is needed and that each resource (e.g. police, firefighters, rescue parties, medical assistance) responds with a different level of mitigation depending on the disaster effect (e.g. building collapse, fire, flood, power loss). While the aforementioned models provide resource allocation schedules following an event, the proposed model allocates resource before the event.

In the model presented by Sathe and Miller-Hooks (2005) the model allocates before the event the emergency response units but the quantity of the units is known, contrary to the SMIP where the number of resources is determined. Their main goal is to locate and re-locate a number of resources in order to cover (provide service) to all the facilities in the network maximizing the facilities with double coverage. Demand must be met since all the critical facilities must be covered by at least one unit. Their 
model considers only one commodity, contrary to the SMIP that considers multiple commodities. The facility locations are fixed, where in the SMIP the facility locations are determined. 


\section{CHAPTER 3. STOCHASTIC MIXED INTEGER PROGRAM}

\subsection{Mathematical Formulation}

Let $G=(N, A, U, C, F, Q)$ be an unreliable multi-source multi-sink flow network defined by a set $N$ of nodes, a set $A$ of directed arcs, a set $U$ of maximum capacities of $A$ that vary with scenario $\mathrm{s} \in S$, a set $C$ of costs per unit of flow on $A$, a set $F$ of fixed unit costs per open facility, and a set $Q$ of unit resource purchase costs. The set $N$ is composed of source, sink and transshipment nodes. Uncertainty in the model is achieved by the use of the set of scenarios. The scenarios include the variability in forecasted demand and the network reliability. The probability of occurrence of the scenario $s$ is represented by the parameter $P_{s}$ where:

$$
\sum_{s \in S} P_{s}=1
$$

Some scenarios considered in the model are obstruction of path segments and destruction of facilities resulting from an extreme event.

The unreliability of the nodes is achieved by dividing each node into two separate twin nodes as shown in Figure 3-1. One node contains the demand and is identified as $n \in N$ while its twin is the supply node and is identified as $n^{\prime} \in N^{\prime} \subset N$. Facilities can be located only on the nodes contained in set $N^{\prime}$. As a default, the links connecting the twin nodes have an infinite capacity and a link cost of zero. In the case when the supply facility contained at node $n$ ' is destroyed due to the natural disaster, the capacity of the link $\left(n^{\prime}, n\right), u_{n^{\prime} n}^{k s}=0$.

Two dummy nodes are connected to the network. These pertain to the excess supply (SE) and unmet demand (UD) as shown in Figure 3-1. These nodes help maintain equilibrium between the amount of demand and supply resources. The 
resource allocated at node UD would be equal to the total difference between the demand and the total allocated resources in the network for each of the commodities. It would thus contain the resource needed for unmet demand. The UD node is connected to the demand nodes $N$. The demand contained in node SE would equal the total unused allocated resource on all the nodes in the network for every commodity $k$ and scenario $s$. The SE node is connected to the source nodes $N^{\prime}$.

Each $\operatorname{arc}(i, j) \in A$ is associated with a maximum capacity $u_{i j}^{k s} \in U$ and a cost $c_{i j}^{k s} \in C$. The unreliability of the network is included in the arc capacities that vary by scenario $s$. Arc costs depend on the nodes connected. The costs incurred for links of source, sinks or transshipment nodes are travel costs. The costs incurred for links leaving the dummy source node are unmet demand penalty costs $p_{i}^{k}$ and for links reaching the dummy sink node are holding costs $h_{i}^{k}$. The links connected to the dummy nodes have infinite capacity. Supply nodes $i$, incur fixed costs $F_{i l}$ corresponding to the opening of a new supply facility depending on its capacity $M_{i l}$, where $l$ is the index pertaining to the different types of facilities that can be chosen. In other words the model will decide if and where to open a facility and also its capacity. The cost incurred for opening a facility of type $l$ of capacity (in terms of volume) $M_{i l}$ at node $i$ is $F_{i l}$.

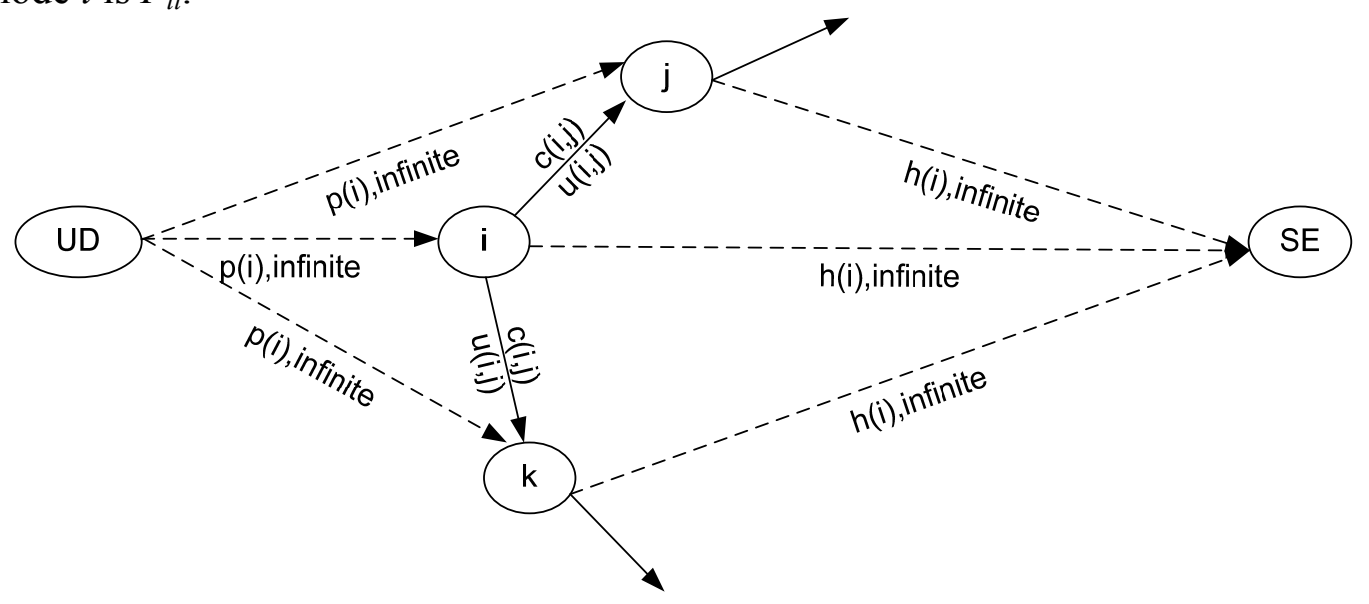

Figure 3-1: Network representation 
Let $y_{i l}$ be a binary decision variable equal to 1 if there is a supplier facility of capacity category $l$ located at node $i, 0$ otherwise; and let $r_{i}^{k}$ be the amount of resource of type $k$ allocated at the supply node. Let $v_{i}^{k s}$ indicate the level of demand for commodity $k$ at the node $i$ in scenario $s$. Let $x_{i j}^{k s}$ be the amount of resource of type $k$ shipped through link $(i, j)$ in scenario $s$.

The objective function minimizes the expected costs over all scenarios resulting from the selection of the supplier locations, the resource purchase and allocation at the supply facilities and the shipments of the supplies to the demand points including the flow in the arcs that represent unmet demand and excess resource.

$$
\min \sum_{i \in N} \sum_{l \in L} F_{l} y_{i l}+\sum_{k \in K} \sum_{i \in N} q_{i}^{k} r_{i}^{k}+\sum_{s \in S} \sum_{(i, j) \in A} \sum_{k \in K} P_{s} c_{i j}^{k s} x_{i j}^{k s}
$$

\section{Subject to:}

(i) Flow conservation

$$
\sum_{j \neq i \in N} x_{j i}^{k s}+r_{i}^{k}=\sum_{j \neq i \in N} x_{i j}^{k s}+v_{i}^{k s} \quad \forall i \in N, k \in K, s \in S
$$

(ii) Arc capacity

$$
x_{i j}^{k s} \leq u_{i j}^{k s} \quad \forall(i, j) \in A, s \in S, k \in K
$$

(iii) Open facilities and facility capacity

$$
\sum_{k \in K} b_{i}^{k} r_{i}^{k} \leq \sum_{l \in L} M_{l} y_{i l} \quad \forall i \in N
$$

(iv) Number of facilities per node

$$
\sum_{l \in L} y_{i l} \leq 1 \quad \forall i \in N
$$

(v) Non-negativity constraints

$$
\begin{array}{ll}
y_{i l} \in(0,1) & \forall i \in N^{\prime}, l \in L \\
r_{i}^{k} \geq 0 & \forall i \in N, k \in K \\
x_{i j}^{k s} \geq 0 & \forall(i, j) \in A, k \in K, s \in S
\end{array}
$$


The first constraint represents the conservation of flow in the network. The second constraint ensures that the link flow does not surpass the arc capacity. In the above formulation maximum link capacity is established per commodity, but this constraint can be modified to restrict the link flow among all commodities traveling through that link. The added modification would cause the commodities to compete for the link capacity like in a multi-commodity flow problem. The third constraint makes certain that resources are assigned to open facilities and that the space taken by these resources $\left(b_{i}^{k}\right)$ does not surpass the facility capacity. The fourth constraint limits the number of open facilities at node $i$ to one. Constraint (v) contains the nonnegativity constraints.

\subsection{Robustness}

Robust optimization (RO), as defined by Mulvey et al. (1995), is a model formulation approach that yield solutions that are less sensitive to variable and uncertain data. This approach combines goal programming with scenario based descriptions of the problem data. These models are composed of two types of decisions variables referred to as design and control variables. The optimal values of the design variables are not conditioned on the realization of the uncertain parameters, while the control variables are subjected to adjustments once the values of the uncertain parameters are known. The first stage and recourse variables of the proposed SMIP model behave in the same manner as design and control variables, respectively. First stage variables determine the structure of the system before the uncertain events occur, i.e. determine the location of source nodes and supplies contained. The recourse 
variables adjust the shipments of the supplies in response to the disruptions in the network, i.e. changes in demand or costs, destruction of facility and supplies.

In robust optimization, the optimal solution of a mathematical program is termed robust with respect to optimality if it remains "close" to optimal for any realization of a scenario $s$. On the other hand, if the solution remains "almost" feasible for any realization of $s$, then the model is referred to as robust. Since it is unlikely that a solution remains both optimal and feasible during all scenarios $s$, a model that measures the tradeoff between solution and model robustness is applied. In this case, to measure the lack of robustness, a penalty function was included that penalizes violations to the control constraints under some scenarios.

Lack of model robustness and feasibility is associated with unmet demand. Penalties for unmet demand are incurred by assigning additional resource, as needed, to the UD dummy node and distributing these resources where requested. These costs and thus, the penalty function are included in the third term of the objective function. The level of unmet demand is equivalent to the total flow emanating from the UD dummy node to all the nodes in the network except to the SE dummy node.

\subsection{Two-Stage Problem}

The SMIP is a stochastic two-stage problem. During the first stage the model determines the location and capacity of the supply facilities $\left(y_{i l}\right)$ and the resource quantity of each commodity $\left(r_{i}^{k}\right)$ to be allocated at each facility. During the second stage the model proceeds to route the resource from the supply facility nodes to the

demand destination nodes $\left(x_{i j}^{k s}\right)$ considering scenario-specific arc capacities $\left(u_{i j}^{k s}\right)$ and $\operatorname{costs}\left(c_{i j}^{k s}\right)$. While the first stage decision variables seek to minimize the average costs 
(including infeasibility penalties) incurred over all scenarios, the second stage decisions variables seek to minimize the costs incurred in every scenario and for every commodity independently, given the first stage decisions.

\subsection{Illustrative Example}

A network of four nodes and eight links illustrates the model formulation. The graphical representation of the network is presented in Figure 3-2. The link transportation costs and capacities are provided in Tables 3-1 and 3-3, respectively. The demand per commodity for each node at each scenario is given in Table 3-2. It was assumed that the link transportation costs were the same for all commodities for simplicity. The fixed costs and storage capacities associated with the storage and distribution facilities are provided in Table 3-4. The unit costs and volume space occupied by each unit of commodity is included in Table 3-5. Three types of resources (commodities) were included: water, food and medicine. Three scenarios were considered that indicate damages incurred by the network and demands for each

commodity resulting from a disaster. The damages resulting from the disaster are reduction in link capacity or in facility capacity and respective resource quantity. A probability of occurrence is assigned to each scenario. As mentioned before in the model formulation, each node is separated into its supply node $n$ ' and its demand node $n$ as shown in Figure 3-2.

Scenarios:

(1) Default: $P(1)=0.3$

(2) The demand at node $\mathrm{A}$ is doubled for all commodities: $P(2)=0.3$ 
(3) The supply facility located at A' is destroyed $\left(u_{A^{\prime}, A}^{k 3}=0, \forall k \in K\right)$ and the capacity on link (B,A) is reduced by $20 \%\left(u_{B, A}^{k 3}=40, \forall k \in K\right): P(3)=0.4$

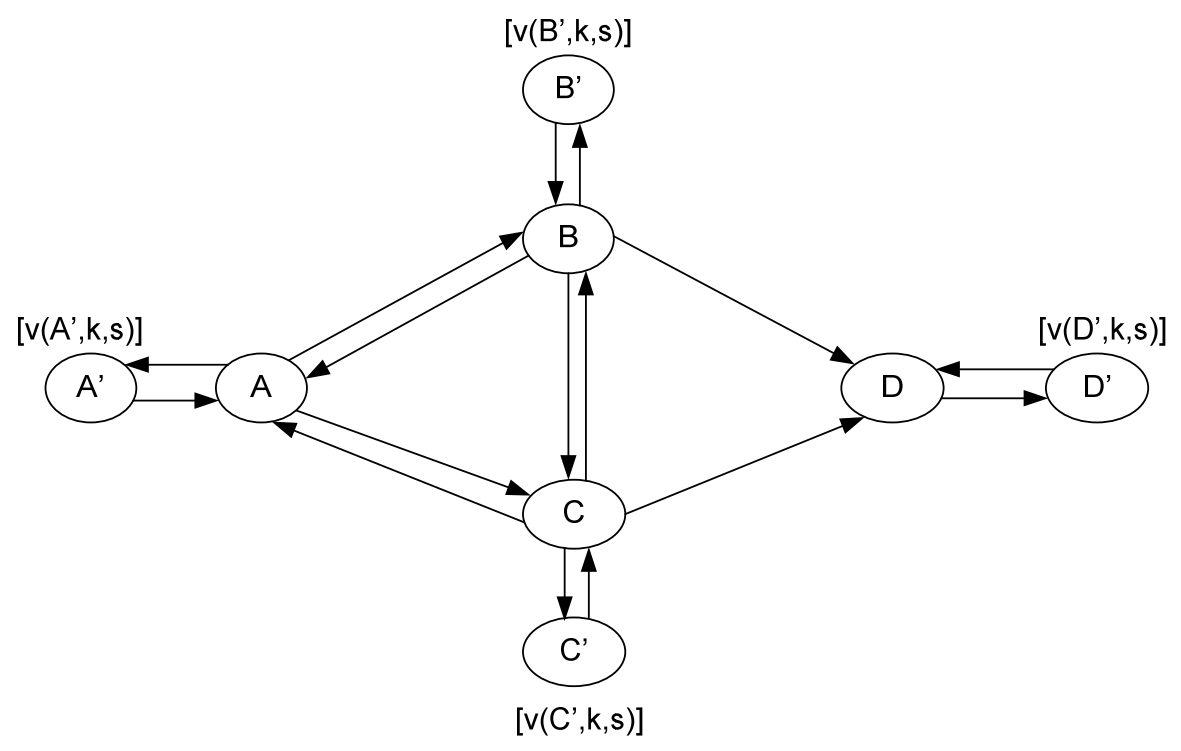

Figure 3-2: Example network

Table 3-1: Link transportation costs, $c_{i j}^{k}$

\begin{tabular}{|c|cccccccccc|}
\hline FROM/TO & $\mathrm{A}$ & $\mathrm{B}$ & $\mathrm{C}$ & $\mathrm{D}$ & $\mathrm{A}^{\prime}$ & $\mathrm{B}^{\prime}$ & $\mathrm{C}^{\prime}$ & $\mathrm{D}^{\prime}$ & $\mathrm{SE}$ & $\mathrm{VE}$ \\
\hline $\mathrm{A}$ & 999 & 10 & 15 & 999 & 0 & 999 & 999 & 999 & 999 & 999 \\
\hline $\mathrm{B}$ & 2 & 999 & 3 & 10 & 999 & 0 & 999 & 999 & 999 & 999 \\
\hline $\mathrm{C}$ & 999 & 8 & 999 & 5 & 999 & 999 & 0 & 999 & 999 & 999 \\
\hline $\mathrm{D}$ & 999 & 999 & 999 & 999 & 999 & 999 & 999 & 0 & 999 & 999 \\
\hline $\mathrm{A}^{\prime}$ & 0 & 999 & 999 & 999 & 0 & 999 & 999 & 999 & 999 & 10 \\
\hline $\mathrm{B}^{\prime}$ & 999 & 0 & 999 & 999 & 999 & 0 & 999 & 999 & 999 & 10 \\
\hline $\mathrm{C}^{\prime}$ & 999 & 999 & 0 & 999 & 999 & 999 & 0 & 999 & 999 & 10 \\
\hline $\mathrm{D}$ & 999 & 999 & 999 & 0 & 999 & 999 & 999 & 0 & 999 & 10 \\
\hline $\mathrm{UD}$ & 50 & 50 & 50 & 50 & 999 & 999 & 999 & 999 & 999 & 0 \\
\hline $\mathrm{SE}$ & 999 & 999 & 999 & 999 & 999 & 999 & 999 & 999 & 999 & 999 \\
\hline
\end{tabular}


Table 3-2: Demand per commodity and scenario, $v_{i}^{k s}$

\begin{tabular}{|c|ccc|ccc|ccc|}
\hline \multirow{2}{*}{ Node } & \multicolumn{3}{|c|}{$\mathrm{s}=1$} & \multicolumn{3}{c|}{$\mathrm{s}=2$} & \multicolumn{3}{c|}{$\mathrm{s}=3$} \\
\cline { 2 - 11 } & Water & Food & Medicine & Water & Food & Medicine & Water & Food & Medicine \\
\hline A & 50 & 10 & 20 & 100 & 20 & 40 & 50 & 10 & 20 \\
\hline B & 50 & 50 & 50 & 50 & 50 & 50 & 50 & 50 & 50 \\
\hline C & 10 & 10 & 10 & 10 & 10 & 10 & 10 & 10 & 10 \\
\hline D & 20 & 20 & 20 & 20 & 20 & 20 & 20 & 20 & 20 \\
\hline
\end{tabular}

Table 3-3: Default link capacity for each commodity $(s=1), u_{i j}^{k s}$

\begin{tabular}{|c|cccccccccc|}
\hline FROM/TO & $\mathrm{A}$ & $\mathrm{B}$ & $\mathrm{C}$ & $\mathrm{D}$ & $\mathrm{A}^{\prime}$ & $\mathrm{B}^{\prime}$ & $\mathrm{C}^{\prime}$ & $\mathrm{D}^{\prime}$ & $\mathrm{SE}$ & $\mathrm{VE}$ \\
\hline $\mathrm{A}$ & 0 & 50 & 50 & 0 & 0 & 0 & 0 & 0 & 0 & 0 \\
\hline $\mathrm{B}$ & 50 & 0 & 50 & 50 & 0 & 0 & 0 & 0 & 0 & 0 \\
\hline $\mathrm{C}$ & 50 & 50 & 0 & 50 & 0 & 0 & 0 & 0 & 0 & 0 \\
\hline $\mathrm{D}$ & 0 & 0 & 0 & 0 & 0 & 0 & 0 & 0 & 0 & 0 \\
\hline $\mathrm{A}^{\prime}$ & 999 & 0 & 0 & 0 & 0 & 0 & 0 & 0 & 0 & 999 \\
\hline $\mathrm{B}^{\prime}$ & 0 & 999 & 0 & 0 & 0 & 0 & 0 & 0 & 0 & 999 \\
\hline $\mathrm{C}^{\prime}$ & 0 & 0 & 999 & 0 & 0 & 0 & 0 & 0 & 0 & 999 \\
\hline $\mathrm{D}$ & 0 & 0 & 0 & 999 & 0 & 0 & 0 & 0 & 0 & 999 \\
\hline $\mathrm{UD}$ & 999 & 999 & 999 & 999 & 0 & 0 & 0 & 0 & 0 & 999 \\
\hline $\mathrm{SE}$ & 0 & 0 & 0 & 0 & 0 & 0 & 0 & 0 & 0 & 0 \\
\hline
\end{tabular}

Table 3-4: Fixed costs incurred with the opening of a warehouse per size and capacities associated with each warehouse size.

\begin{tabular}{|cccc|}
\hline & Small & Medium & Large \\
\hline $\mathrm{F}$ & 100 & 200 & 300 \\
\hline $\mathrm{M}$ & 100 & 500 & 1000 \\
\hline
\end{tabular}

Table 3-5: Purchase cost and volume space occupied per unit of commodity

\begin{tabular}{|cccc|}
\hline & Water & Food & Medicine \\
\hline Q (\$/unit) & 10 & 20 & 50 \\
\hline B (volume/unit) & 10 & 5 & 1 \\
\hline
\end{tabular}

This problem was small enough, with 24 first stage and 72 second stage variables, to be solved in extensive form using an integer program solver. However, the number of variables increases exponentially with respect to the number of scenarios, nodes, commodities and facility types making it impossible for a standard 
integer program solver to tackle a large-scale version. For this reason, a heuristic method was developed and is presented in section 4.4.

\section{$\underline{\text { Solution }}$}

Objective value: 23090.40

Table 3-6: Facility location and size with its respected allocated resource

\begin{tabular}{|c|c|c|c|c|c|}
\hline & & \multicolumn{3}{|c|}{$\mathrm{R}$} & \multirow{2}{*}{$\begin{array}{c}\text { Total Volume } \\
\text { Stored }\end{array}$} \\
\cline { 3 - 5 } Node & Y(size) & Water & Food & Medicine & 670 \\
\hline A $^{\prime}$ & Medium & 60 & 10 & 20 & 1000 \\
\hline B' $^{\prime}$ & Large & 63 & 60 & 70 & 430 \\
\hline C' $^{\prime}$ & Large & 37 & 10 & 10 & 320 \\
\hline D' $^{\prime}$ & Medium & 20 & 20 & 20 & \\
\hline
\end{tabular}

Table 3-6 presents the optimal solution found. One facility is opened in each node, two facilities of medium capacities and two of large capacities. The nodes with medium facilities are those than are not able to ship supplies because they consume the supplies (node A') or because they do not have any emanating arcs (node D'). In terms of resources, the solution allocates enough medicine and food to satisfy the total demand of all the nodes in the network regardless of the holding costs. An example is shown in scenario 1 where nodes A', B' and C' incur holding costs. Note that the penalties for unmet demand are five times the holding costs, so the model will be more conservative purchasing the supplies. In terms of meeting the demand for each node, the solution allocated enough water, food, and medicine to satisfy the needs of nodes B, C, and D in all the scenarios. The total amount of resources allocated in node B' was limited by the maximum warehouse capacity.

The situation at node A is different and should be discussed separately. Enough water was allocated at node $A^{\prime}$ to satisfy its demand fully $70 \%$ of the time, not 
meeting $20 \%$ of its demand $40 \%$ of the time. Only a fraction of the demand for water at node A could be satisfied when the supply facility at node A' was destroyed, because of the following reasons:

- Node B transported as much material as possible because it is its nearest neighbor. The amount of water resource sent from B' was limited by the maximum allowable warehouse capacity. Priority for resource purchase was given to food and medicine which have lower volume and can therefore permit more demand to be met.

- The amount of resource sent from node C', on the other hand, was limited by the path capacity C-B-A, since the resource coming from C' shared the link capacity with the resource coming from B'. No supplies could be shipped directly from C' to A because the shipping costs are higher than the unmet demand penalty costs.

Decision: In scenario 1 node A consumes all the resources allocated in node $\mathrm{A}^{\prime}$. In scenarios 2 and 3 , nodes $\mathrm{B}$ and $\mathrm{C}$ send node $\mathrm{A}$ all excess resources they have available. However, in scenario 3 the amount of water sent to $\mathrm{A}$ is not enough to satisfy its demand completely. The detailed scheme of resource distribution per scenario is presented below.

\section{$\underline{\text { Resource distribution per scenario }}$}

The quantities of demand and supply are presented in vector form as follows:

$$
\left[\begin{array}{l} 
\pm \text { Water } \\
\pm \text { Food } \\
\pm \text { Medicine }
\end{array}\right]
$$

where the minus sign indicates demand and the plus sign indicates supply. The characters as defined previously, were located in front of the vectors to indicate their origin and correspondence. 
Scenario $=1$

Node A: $v_{A}\left[\begin{array}{l}-50 \\ -10 \\ -20\end{array}\right]+r_{A^{\prime}}\left[\begin{array}{c}60 \\ 10 \\ 20\end{array}\right]=\left[\begin{array}{l}10 \\ 0 \\ 0\end{array}\right]$

Node B: $v_{B}\left[\begin{array}{l}-50 \\ -50 \\ -50\end{array}\right]+r_{B^{\prime}}\left[\begin{array}{l}63 \\ 60 \\ 70\end{array}\right]=\left[\begin{array}{l}13 \\ 10 \\ 20\end{array}\right]$

Node $C: v_{C}\left[\begin{array}{l}-10 \\ -10 \\ -10\end{array}\right]+r_{C^{\prime}}\left[\begin{array}{l}37 \\ 10 \\ 10\end{array}\right]=\left[\begin{array}{l}27 \\ 0 \\ 0\end{array}\right]$

Node D: $v_{D}\left[\begin{array}{l}-20 \\ -20 \\ -20\end{array}\right]+r_{D^{\prime}}\left[\begin{array}{l}20 \\ 20 \\ 20\end{array}\right]=\left[\begin{array}{l}0 \\ 0 \\ 0\end{array}\right]$

In scenario 1, 50 units of water, 10 units of food and 20 units of medicine incur holding costs.

Scenario $=2$

Node A: $v_{A}\left[\begin{array}{l}-100 \\ -20 \\ -40\end{array}\right]+r_{A^{\prime}}\left[\begin{array}{c}60 \\ 10 \\ 20\end{array}\right]+x_{B, A}\left[\begin{array}{c}40 \\ 10 \\ 20\end{array}\right]=\left[\begin{array}{l}0 \\ 0 \\ 0\end{array}\right]$

Node B: $v_{B}\left[\begin{array}{l}-50 \\ -50 \\ -50\end{array}\right]+r_{B^{\prime}}\left[\begin{array}{l}63 \\ 60 \\ 70\end{array}\right]+x_{B, A}\left[\begin{array}{l}-40 \\ -10 \\ -20\end{array}\right]+x_{C, B}\left[\begin{array}{l}27 \\ 0 \\ 0\end{array}\right]=\left[\begin{array}{l}0 \\ 0 \\ 0\end{array}\right]$

Node C: $v_{C}\left[\begin{array}{l}-10 \\ -10 \\ -10\end{array}\right]+r_{C^{\prime}}\left[\begin{array}{l}37 \\ 10 \\ 10\end{array}\right]+x_{C, B}\left[\begin{array}{l}-27 \\ 0 \\ 0\end{array}\right]=\left[\begin{array}{l}0 \\ 0 \\ 0\end{array}\right]$

Node D: $v_{D}\left[\begin{array}{l}-20 \\ -20 \\ -20\end{array}\right]+r_{D^{\prime}}\left[\begin{array}{l}20 \\ 20 \\ 20\end{array}\right]=\left[\begin{array}{l}0 \\ 0 \\ 0\end{array}\right]$ 
In scenario 2, no holding or unmet demand penalty costs were incurred. The demand at node A was met sending all the excess resource allocated at nodes B and C. Scenario $=3$

Node A: $v_{A}\left[\begin{array}{l}-50 \\ -10 \\ -20\end{array}\right]+r_{A^{\prime}}\left[\begin{array}{l}0+60 \\ 0+10 \\ 0+20\end{array}\right]+x_{B, A}\left[\begin{array}{l}40 \\ 10 \\ 20\end{array}\right]=\left[\begin{array}{l}-10+60 \\ 0+10 \\ 0+20\end{array}\right]$

Node B: $v_{B}\left[\begin{array}{l}-50 \\ -50 \\ -50\end{array}\right]+r_{B^{\prime}}\left[\begin{array}{l}63 \\ 60 \\ 70\end{array}\right]+x_{B, A}\left[\begin{array}{l}-40 \\ -10 \\ -20\end{array}\right]+x_{C, B}\left[\begin{array}{l}27 \\ 0 \\ 0\end{array}\right]=\left[\begin{array}{l}0 \\ 0 \\ 0\end{array}\right]$

Node $C: v_{C}\left[\begin{array}{l}-10 \\ -10 \\ -10\end{array}\right]+r_{C^{\prime}}\left[\begin{array}{l}37 \\ 10 \\ 10\end{array}\right]+x_{C, B}\left[\begin{array}{l}-27 \\ 0 \\ 0\end{array}\right]=\left[\begin{array}{l}0 \\ 0 \\ 0\end{array}\right]$

Node D: $v_{D}\left[\begin{array}{l}-20 \\ -20 \\ -20\end{array}\right]+r_{D^{\prime}}\left[\begin{array}{l}20 \\ 20 \\ 20\end{array}\right]=\left[\begin{array}{l}0 \\ 0 \\ 0\end{array}\right]$

In scenario 3, due to the destruction of the storage facility located at node A', which had 60 units of water, 10 units of food, and 20 units of medicine; resource purchase and loss costs, were incurred for the destroyed goods. Note that since the facility was destroyed, the resources were lost and 10 of units of water incurred penalty costs for unmet demand at node A.

This example was devised in order to show the properties of the SMIP formulation described in this chapter. The example shows the model robustness by providing a solution that balances the level of unmet demand and of excess resource. Based on the magnitude of the penalty costs, the model decides to purchase supplies that cannot be shipped through the network (due to link capacity restraints) in some instances incurring holding costs, so as not to decrease the level of satisfied demand even further. This example also shows the information included in the scenarios such 
as network reliability and changes in demand levels. Finally, it shows how by weighting all the information provided in the scenarios, the facility location and quantities of pre-positioned resources were determined. This last provides insight on how this example can be expanded in order to tackle a more detailed and realistic problem. 


\section{CHAPTER 4. METHODOLOGY}

The main goal of the model presented in Chapter 3 is to provide a prepositioning strategy planning tool to determine the most advantageous a priori resource allocation scheme in order to assist disaster victims with minimum response time. An illustrative resource pre-positioning plan, resulting from solving the SMIP in extensive form, was presented in section 3.4. The plan showed how the model determined the best resource locations based on probable scenarios that include both damages in the network and demands arising as a result of the disaster. The past example with only four nodes and eight links had to find the values for 24 first stage and 72 second stage variables, where half of the first stage variables are binary. The number of variables of the SMIP formulation grows exponentially with the number of cities in the network, facility types, commodities and scenarios considered.

The methodology explained in this chapter offers computationally attractive solutions for the NP-hard problem at hand, making it possible for large scale prepositioning plans to be devised. This methodology decomposes the SMIP into smaller, easier to solve sub-problems. This is done by combining two techniques: the L-shaped method and the Lagrangian relaxation method. The L-shaped method developed by Van Slyke and Wets (1969) and also contained in Birge and Louveaux (1997), offers an overall framework for the problem solution. Within this framework, the second stage problem is a set of minimum cost flow problems, one for each scenariocommodity combination. Given the amount of resources allocated, the sources and the demand node locations, the algorithm finds the least cost paths to send the supply shipments without violating the link capacity constraints. This special structure contributes to an ability to solve the scenario-specific second stage sub-problems 
rapidly and efficiently. An important aspect of the L-shaped method is that it takes advantage of the computational attractiveness of complete recourse problems.

The Lagrangian relaxation method as described in section 4.3 is used to relax the complicating facility capacity constraint and as a result, decompose the first stage problem into a trivial integer problem and a resource allocation linear program. These are two sub-problems within the L-shaped method decomposition that are computationally inexpensive. An additional benefit of this strategy is that the integrality constraints of the binary variable $\left(y_{i l}\right)$ are relaxed. This further improves the computational capabilities of the algorithm. Previous work, the Integer L-shaped method published by Laporte and Louveaux (1993), relaxed the integrality constraints by adding branch and cut techniques to the L-shaped method of Van Slyke and Wets (1969).

The following sections describe how the SMIP formulation is decomposed and how these sub-problems solutions fit into the larger framework of the L-shaped method for the overall problem. First, section 4.1 includes an in-depth presentation of the overall framework and how the L-shaped method divides the SMIP into its first stage and second stage problems. Section 4.2 follows with the description of the second stage sub-problems, the network flow problems, whose computational ease is exploited by the L-shaped methodology. Section 4.3 explains how the Lagrangian relaxation was used to decompose the first stage problem into more computationally tractable problems. Finally, section 4.4 introduces the algorithm resulting from the combination of the L-shaped method and the Lagrangian relaxation techniques, referred to as the Lagrangian L-shaped method (LLSM). The flow chart of the LLSM is shown in Figure 4-4. 


\subsection{Master Problem: L-shaped Method}

The L-shaped method, developed by Van Slyke and Wets (1969), avoids the numerous function evaluations of second stage recourse linear programs by approximating the recourse function. The L-shaped method consists of solving an approximation of a stochastic program by using an outer linearization of $H(y, r)$, the expected value of the second stage problem (Birge and Louveaux, 1997). Outer linearization is an approximation scheme used for nonlinear programs that approximates a function by using its slopes (Bradley et al., 1977) as shown in Figure 4-1. The light dashed lines represent the constraints referred to as optimality cuts that will approximate $H(y, r)$ by $\theta$, represented in the bold dashed lines.

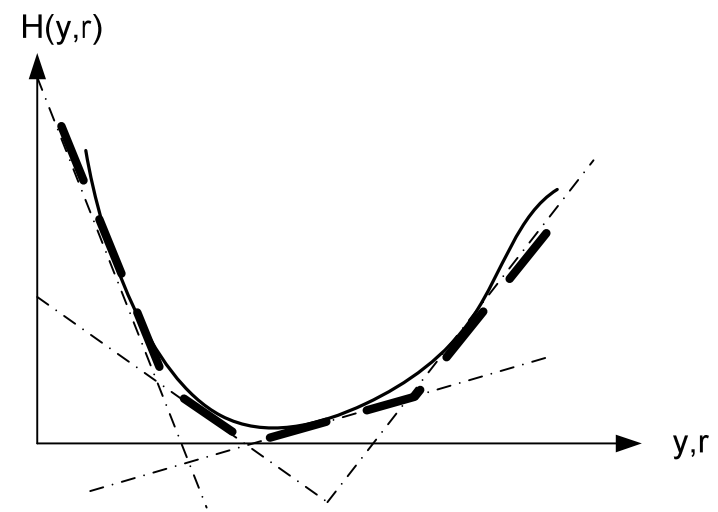

Figure 4-1: Outer linearization for convex function

During the operation of the L-shaped method, two types of constraints are added: feasibility cuts that assure the feasibility of the solution for the second stage problem and optimality cuts, which are linear approximations of $H(y, r)$. However, since the problem to be solved here has complete recourse (i.e. there is always a feasible second stage solution), any feasible solution resulting from the first stage problem will be feasible for the second stage problem regardless of the scenario or the commodity. Thus, only optimality cuts need to be added. 
At every stage of the algorithm, the following current problem (CP) is considered. The current problem is obtained from the stochastic mixed integer program by relaxing the exact definition of $H(y, r) . H(y, r)$ is relaxed in a polyhedral representation by $\theta$ and the constraints called optimality cuts (refer to Figure 4-1).

(CP) $\min \sum_{i \in N} \sum_{l \in L} F_{i l} y_{i l}+\sum_{i \in N} \sum_{k \in K} q_{i}^{k} r_{i}^{k}+\theta$

\section{Subject to:}

(i) Open facilities and facility capacity

$$
\sum_{k \in K} b_{i}^{k} r_{i}^{k} \leq \sum_{l \in L} M_{i l} y_{i l} \quad \forall i \in N^{\prime}
$$

(ii) At most one facility per node

$$
\sum_{l \in L} y_{i l} \leq 1 \quad \forall i \in N^{\prime}
$$

(iii) Optimality cuts

$$
E_{\chi} r+\theta \geq e_{\chi} \quad \forall \chi \in \mathrm{X}
$$

(iv) Non-negativity constraints

$$
\begin{array}{ll}
y_{i l} \geq 0 & \forall i \in N^{\prime}, l \in L \\
r_{i}^{k} \geq 0 & \forall i \in N^{\prime}, k \in K
\end{array}
$$

\section{Variables:}

Let $\mathrm{z}$ be the current objective value and $z *$ be the estimated optimal objective value.

Let $\delta$ be the current solution number.

Let $H(y, r)$ be the expected value of the second stage problem:

$$
H(y, r)=\sum_{s \in S} \sum_{(i, j) \in A} \sum_{k \in K} P_{s} c_{i j}^{k s} x_{i j}^{k s}
$$

as given by the $k$ network flow models.

Let $\theta$ be the approximate value of $H(y, r)$ where $\theta \leq H(y, r)$.

Let $\chi$ be the optimality cut number. Optimality cuts are supportive hyperplanes of $H(y, r)$. These are obtained from the following relationship: $\theta \geq e_{\chi+1}-E_{\chi+1} r_{i}^{k \delta}$ 
where $E_{\chi+1}=\sum_{s \in S} \sum_{i \in N} \sum_{k \in K}\left[p_{s}\left(w_{i}^{k s \delta}\right)\right]$ and $e_{\chi+1}=\sum_{s \in S} \sum_{i \in N} \sum_{k \in K}\left[p_{s}\left(w_{i}^{k s \delta}\right) v_{i}^{k s}\right]$.

The optimality cuts are based on dual theory in linear programming. At every iteration $\delta$, a minimum cost flow problem is solved to optimality for every scenario and every commodity yielding the simplex multiplier shadow prices associated with the flow conservation constraint $\left(w_{i}^{k s \delta}\right)$. By weak duality at an arbitrary iteration $\delta$ for scenario $s$ and commodity $k$, for a feasible solution the following relationship is true: $H(y, r, s) \geq\left(w_{i}^{k s \delta}\right)\left(v_{i}^{k s}-r_{i}^{k \delta}\right)$.

Taking the expected value, the following is obtained:

$$
\begin{aligned}
& H(y, r) \geq E\left[\left(w_{i}^{k s \delta}\right)\left(v_{i}^{k s}-r_{i}^{k \delta}\right)\right] \\
& =\sum_{s \in S}\left[P_{s}\left(w_{i}^{k s \delta}\right)\left(v_{i}^{k s}-r_{i}^{k \delta}\right)\right] \\
& =\sum_{s \in S}\left[P_{s}\left(w_{i}^{k s \delta}\right)\left(v_{i}^{k s}\right)\right]-\sum_{s \in S}\left[P_{s}\left(w_{i}^{k s \delta}\right)\left(r_{i}^{k \delta}\right)\right]
\end{aligned}
$$

Note that to solve the SMIP is equivalent to solving:

$$
\min \sum_{i \in N^{\prime}} \sum_{l \in L} F_{i l} y_{i l}+\sum_{i \in N^{\prime}} \sum_{k \in K} q_{i}^{k} r_{i}^{k}+\theta
$$

Subject to:

(i) Open facilities and facility capacity

$\sum_{k \in K} b_{i}^{k} r_{i}^{k} \leq \sum_{l \in L} M_{l} y_{i l} \quad \forall i \in N$

(ii) Number of facilities per node

$$
\sum_{l \in L} y_{i l} \leq 1 \quad \forall i \in N
$$

(iii) Approximation gap

$$
H(y, r)=\sum_{s \in S} \sum_{k \in K} \sum_{(i, j) \in A} P_{s} c_{i j}^{k s} x_{i j}^{k s} \geq \theta
$$

(iv) Non-negativity constraints

$$
\begin{array}{ll}
y_{i l} \in(0,1) & \forall i \in N^{\prime}, l \in L \\
r_{i}^{k} \geq 0 & \forall i \in N^{\prime}, k \in K
\end{array}
$$


Since $\theta$ is unrestricted save for constraint (iii), then it follows that for a $(r, \theta)$ solution pair to be feasible $\theta \geq H(y, r)$ and so, $\theta \geq E\left(w_{i}^{k s}\right)^{\delta}\left(v_{i}^{k s}-r_{i}^{k \delta}\right)$ which is the third constraint of the CP. At optimality $\theta=H(y, r)$ since $\theta$ is unrestricted save for $\theta \geq H(y, r)$. Thus, at each iteration either $\theta=H(y, r)$ and an optimal solution has been obtained or $\theta<H(y, r)$ and the optimality cuts that have been created previously have not been able to adequately define the relationship $\theta \geq H(y, r)$, and therefore a new optimality cut must be added. Following is the L-shaped method procedure.

\section{General procedure}

Step0. Set $\chi=\delta=0, z^{*}=\infty$. Set $\theta=-\infty$ and ignore during the initial computation.

Step1. Set $\delta=\delta+1$. Solve the current problem (CP). Set $\left(y^{\delta}, r^{\delta}, \theta^{\delta}\right)$ as the current optimal solution.

Step2. Check the value of the current solution: if $F^{T} y^{\delta}+q^{T} r^{\delta}+\theta^{\delta}>z^{*}$ then fathom the current problem (fathom by bounds) and go to Step1.

Step3. Compute $H\left(y^{\delta}, r^{\delta}\right)$ and $z^{\delta}=F^{T} y^{\delta}+q^{T} r^{\delta}+H\left(y^{\delta}, r^{\delta}\right)$. If $z^{\delta}<z^{*}$ then update $z^{*}=z^{\delta}$.

Step4. If $\theta^{\delta} \geq H\left(y^{\delta}, r^{\delta}\right)$ then fathom the current node (fathom by optimality cut) and end. Otherwise, impose one optimality cut, set $\chi=\chi+1$ and return to Step2.

\section{Example}

Following is an illustrative example that shows how the optimality cuts are generated. The problem is a pre-positioning strategy with only two cities and one 
commodity (water). The resource purchase price and volume occupied for water are $q_{i}^{\text {water }}=\$ 10$ per thousand gallons and $b_{i}^{\text {water }}=200 \mathrm{ft}^{3}$ per thousand gallons, respectively. In this case, three facility types are included (e.g. small, medium and large). The operating costs for the these facilities are $\left(F_{i, \text { small }}=100, F_{i, \text { medium }}=200\right.$, and $\left.F_{i, \text { arge }}=300\right)$ and their maximum storage capacities are $\left(M_{i, \text { small }}=2000\right.$, $M_{i, \text { medium }}=10000$, and $\left.M_{i, \text { arge }}=20000\right)$. City A has a demand of 38 units of commodity $\left(v_{A}^{k s}=38\right)$ while city B has a demand of 23 units $\left(v_{B}^{k s}=23\right)$. The link costs are: $c_{A, B}=10$ and $c_{B, A}=2$. Link capacities between cities were set to 50 units $\left(u_{A, B}=u_{B, A}=50\right)$. The unmet demand unit penalty costs are equal to $\$ 50$ and the unit holding costs are $\$ 10$. The SMIP formulation presented in chapter 3 is used and the current problem formulation is as follows.

$$
\min \sum_{i=A^{\prime}, B^{\prime} l} \sum_{l=s, m, l} F_{i l} y_{i l}+\sum_{i=A^{\prime}, B^{\prime}} \sum_{k=\text { water }} q_{i}^{k} r_{i}^{k}+\theta
$$

\section{Subject to:}

(i) Open facilities and facility capacity

$$
\sum_{k \in K} b_{i}^{k} r_{i}^{k} \leq \sum_{l \in L} M_{l} y_{i l} \quad \forall i \in N
$$

(ii) Number of facilities per node

$$
\sum_{l \in L} y_{i l} \leq 1 \quad \forall i \in N
$$

(iii) Non-negativity constraints

$$
\begin{array}{ll}
y_{i l} \in(0,1) & \forall i \in N^{\prime}, l \in L \\
r_{i}^{k} \geq 0 & \forall i \in N^{\prime}, k \in K
\end{array}
$$

Table 4-1 includes the results obtained at each iteration for the current problem objective $(z)$, the recourse function $(H(y, r))$, the estimated second stage cost $(\theta)$, and the first stage variables $\left(y_{i l}\right.$ and $\left.r_{i}^{k}\right)$. 
Table 4-1: Values per iteration of the first and second stage problems

\begin{tabular}{|c|c|c|c|c|c|c|c|}
\hline iteration & $\mathrm{H}(\mathrm{y}, \mathrm{r})$ & Theta & $\mathrm{Z}$ & $\mathrm{Y}(\mathrm{A})$ & $\mathrm{Y}(\mathrm{B})$ & $\mathrm{R}(\mathrm{A})$ & $\mathrm{R}(\mathrm{B})$ \\
\hline 0 & 3050 & -99999 & 0 & 0 & 0 & 0 & 0 \\
\hline 1 & 230 & 0 & 361 & Large & 0 & 61 & 0 \\
\hline 2 & 76 & 0 & 361 & 0 & Large & 0 & 61 \\
\hline 3 & 104 & 56 & 415.88 & Small & Medium & 10 & 49.88 \\
\hline 4 & 76 & 76 & 437 & 0 & Large & 0 & 61 \\
\hline
\end{tabular}

Figure 4-2 shows a graphical representation of how the recourse function is approximated with the addition of the optimality cuts during the outer-linearization process. An explanation of how these optimality cuts are generated is presented below.

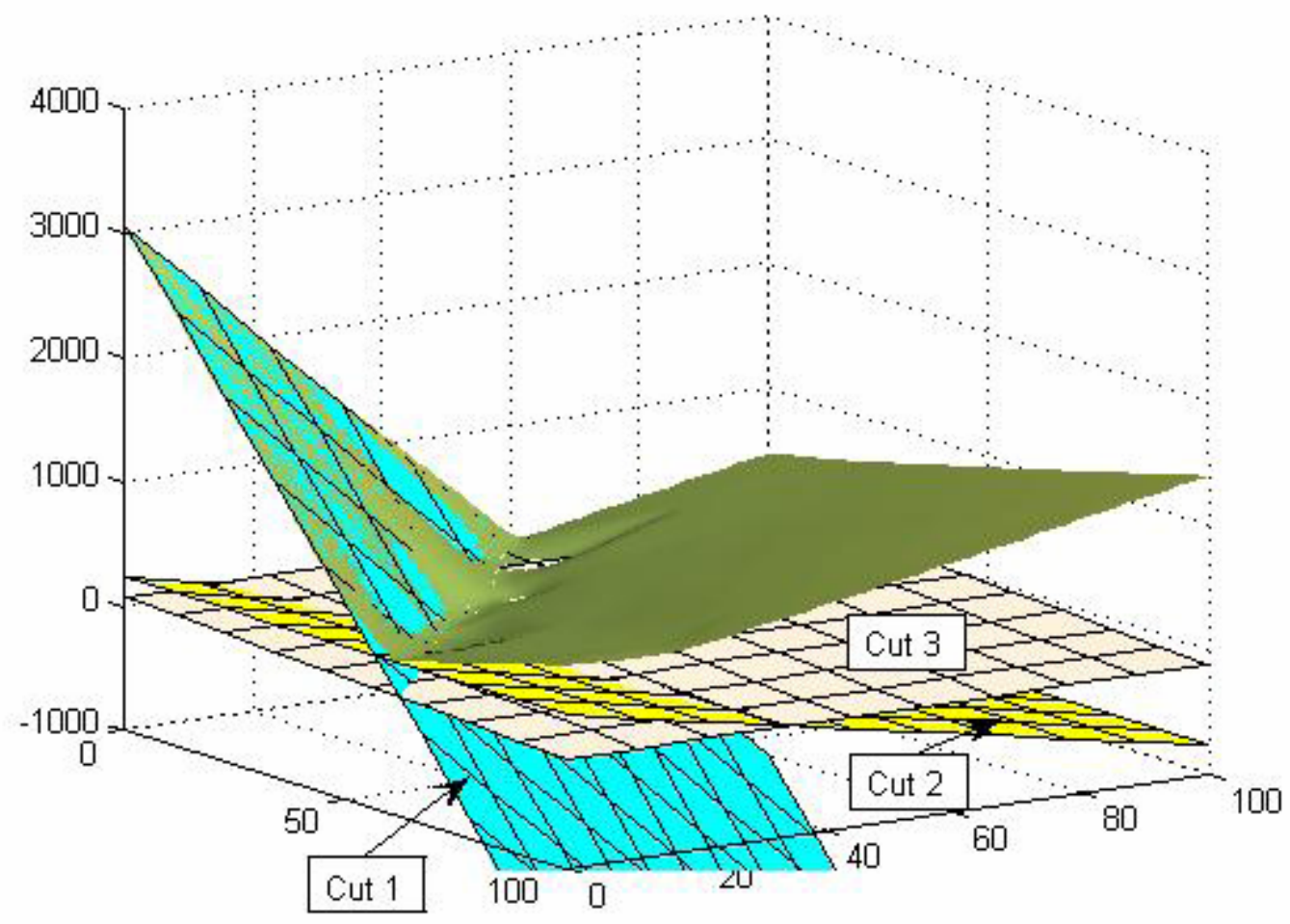

Figure 4-2: Second stage function and optimality cuts 
At iteration 0, all variables are initialized: $\theta=-\infty, y_{i, l}=0 \quad \forall i \in N, l \in L$, $r_{i}^{k}=0 \forall k \in K, i \in N, \mathrm{z}=0$. Since there are no resources allocated in the system, the unmet demand of each city incurs penalty costs equaling a value of 3050 $(=50 *(38+23))$. The latter is the initial recourse function value. Since $\theta<H(y, r)$ and $\theta$ is an outer-linearization of $H(y, r)$, an optimality cut of the form

$$
\theta \geq \sum_{s \in S} \sum_{(i, j) \in A} \sum_{k \in K} P_{s} w_{i j}^{k s} v_{i}^{k s}-\sum_{s \in S} \sum_{(i, j) \in A} \sum_{k \in K} P_{s} w_{i j}^{k s} r_{i}^{k}
$$

must be added as a constraint to the current problem. With the shadow prices equal to $50\left(w_{A}=50, w_{B}=50\right)$, the resulting optimality cut is:

$$
\theta \geq 50(38)+50(23)-50 * r_{A}-50 * r_{B}
$$

After solving the resulting $\mathrm{CP}, H(y, r)=230$ and $\theta=0<H(y, r)$ so a new optimality cut must be added to the current problem. The shadow prices in this case are 0 for node $\mathrm{A}$ and 10 for node $\mathrm{B}\left(w_{A}=0, w_{B}=10\right)$. The optimality cut is equal to:

$$
\theta \geq 10(23)-10 * r_{B}
$$

This added constraint produces a value of 76 for the recourse function while the estimated value stays with a value of zero $(\theta=0<H(y, r)=76)$. The shadow prices in this iteration are $w_{A}=2$ and $w_{B}=0$. The new optimality cut is:

$$
\theta \geq 2(38)-2 * r_{A}
$$

Solving the current problem produces a value of 104 for the recourse function and a value of 56 for the estimated second stage cost. Since $\theta=56<H(y, r)=104$, a new optimality cut must be added. With the shadow prices of $w_{A}=50$ and $w_{B}=48$, the optimality cut becomes:

$$
\theta \geq 50(38)+48(23)-50 * r_{A}-48 * r_{B}
$$

The recourse function and the estimated recourse function values obtained after solving the current problem are 76 each. Since they are equal, we reached an optimal solution. As shown in Table 4-1, a large facility is opened in node B' with 61 stored units of commodity (e.g. water). 


\subsection{Sub-problem: Minimum Cost Flow Problem}

The second stage problems of the SMIP formulation are minimum cost flow problems. Minimum cost flow problems determine the least cost shipment of a commodity through a network in order to satisfy the demands at certain nodes from available supplies at other nodes while considering the arc capacities (Ahuja et al., 1993). In this case, once the facility locations have been determined and the resources have been allocated, the recourse problem ships each commodity through the network towards the demand points in each scenario. The reasoning behind this decomposition is defined next.

The first term of the objective function of the SMIP as presented in section 3.1,

$$
\sum_{s \in S} \sum_{(i, j) \in \in A} \sum_{k \in K} P_{s} c_{i j}^{k s} x_{i j}^{k s}
$$

can be represented as

$$
\sum_{s \in S} \sum_{k \in K} P_{s}\left[z^{k s}\left(r^{k}\right)\right]
$$

where $z^{k s}\left(r^{k}\right)$ is the value of the objective function for the minimum cost flow problem for commodity $k$ in scenario $s$. For any fixed value of the resource variables $r_{i}^{k}$ the problem decomposes into $k$ separate minimum cost flow sub-problems for each scenario.

$z^{k s}\left(r^{k}\right)=\min \sum_{(i, j) \in A} c_{i j}^{k s} x_{i j}^{k s}$

Subject to:

(i) Flow conservation

$\sum_{j \neq i \in N} x_{j i}^{k s}+r_{i}^{k}=\sum_{j \neq i \in N} x_{i j}^{k s}+v_{i}^{k s} \quad \forall i \in N, k \in K, s \in S$

(ii) Arc capacity

$x_{i j}^{k s} \leq u_{i j}^{k s} \quad \forall(i, j) \in A, k \in K, s \in S$

(iii) Non-negativity

$x_{i j}^{k s} \geq 0 \quad \forall(i, j) \in A, k \in K, s \in S$ 
Then $\sum_{s \in S} \sum_{k \in K} P_{s}\left[z^{k s}\left(r^{k}\right)\right]=E\left[z^{k s}\left(r^{k}\right)\right]$ is the expected value of the objective

function over all commodities and all scenarios. The solution also produces $w_{i}^{k s}$, the shadow prices obtained at optimality for the flow conservation constraints in the network flow problems (by commodity and by scenario). They represent the marginal costs associated with a unit change of resource $r_{i}^{k}$. These dual variables play a direct role in the construction of the optimality cuts of the L-shaped method for the overall problem.

There are many algorithms available designed to solve minimum cost flow problems. Some like the cycle-canceling algorithms, the successive shortest path algorithms, the primal-dual and the out-of-kilter algorithms; solve a sequence of shortest path problems with respect to maximum flow residual networks and augmenting paths. All these algorithms have pseudo-polynomial running times. An algorithm with better running times used to solve minimum cost flow problems is the network simplex algorithm, which is an adaptation of the well known simplex method for linear programs. In the case of the minimum cost flow problem, the linear programming basis is a spanning tree. At every iteration, the network simplex moves from one spanning tree solution to another until it finds a spanning tree that satisfies the network optimality conditions. The latter was chosen to solve the second stage minimum cost flow sub-problems. This operation is realized during the third step of the L-shaped method as described in the previous section.

\subsection{Sub-problem: Lagrangian Relaxation}

A Lagrangian relaxation approach was used to solve the CP of the L-shaped method as described in section 4.1. The Lagrangian relaxation procedure is based on 
the observation that many difficult integer programs can be modeled as relatively easy core problems with a small number of relatively difficult constraints (Fisher, 1985). These constraints are relaxed and their absence from the feasible region is included in the objective function as a penalty term with associated Lagrange multipliers $\mu$. In this case, the constraint to be relaxed is constraint (i) of the CP shown in section 4.1 pertaining to the opening of new facilities and to the resource allocation based on the facility's capacity. This constraint was chosen because without it, the problem (CP) decomposes into two separate sub-problems - one in the $y_{i l}$ variables and the other in the $r_{i}^{k}$ variables. These sub-problems can be solved very easily. The resulting Lagrangian relaxation $L(\mu)$ of the original CP is as follows:

$L(\mu)=\min \sum_{i \in N} \sum_{l \in L} F_{i l} y_{i l}+\sum_{i \in N} \sum_{k \in K} q_{i}^{k} r_{i}^{k}+\theta+\sum_{i \in N} \mu_{i}\left\{\sum_{k \in K} b_{i}^{k} r_{i}^{k}-\sum_{l \in L} M_{i l} y_{i l}\right\}$

Subject to:

(i) At most one facility per node

$\sum_{l \in L} y_{i l} \leq 1 \quad \forall i \in N^{\prime}$

(ii) Optimality cuts

$E_{\chi} r+\theta \geq e_{\chi} \quad \forall \chi \in \mathrm{X}$

(iii) Non-negativity constraints

$y_{i l} \in(0,1) \quad \forall i \in N^{\prime}, l \in L$

$r_{i}^{k} \geq 0 \quad \forall i \in N^{\prime}, k \in K$

As a result of the Lagrangian relaxation, for fixed values of $\mu_{i}$ the $\mathrm{CP}$ is decomposed into a trivial integer program (SP1) and a resource allocation linear program (SP2). 
(SP1) $\min \sum_{i \in N} \sum_{l \in L}\left(F_{i l}-\mu_{i} M_{i l}\right) y_{i l}$

Subject to:

(i) At most one facility per node

$\sum_{l \in L} y_{i l} \leq 1 \quad \forall i \in N^{\prime}$

(ii) Non-negativity constraints

$$
y_{i l} \in(0,1) \quad \forall i \in N^{\prime}, l \in L
$$

(SP2) $\min \sum_{i \in N} \sum_{k \in K}\left(q_{i}^{k}+\mu_{i} b_{i}^{k}\right) r_{i}^{k}+\theta$

Subject to:

(i) Optimality cuts

$$
E_{\chi} r+\theta \geq e_{\chi} \quad \forall \chi \in \mathrm{X}
$$

(ii) Non-negativity constraints

$$
r_{i}^{k} \geq 0 \quad \forall i \in N^{\prime}, k \in K
$$

The greedy algorithm shown in Figure 4-3 will open facilities to location $i \in N^{\prime}$ only where the $y_{i l}$ coefficient $\left(F_{i l}-\mu_{i} M_{i l}\right)$ has the most negative value among all facility sizes per node. Facilities of a specific magnitude will be opened only in the locations for which the shadow price on the facility capacity constraint is larger than the fixed cost of the facility.

The second sub-problem (SP2) is a linear program that determines the optimal quantities of resource of different commodities based on their purchase price and on how much storage space they occupy or require, while considering their shipment costs and the penalties incurred when unavailable. The solution for SP2 is obtained with a commercial linear problem solver. 


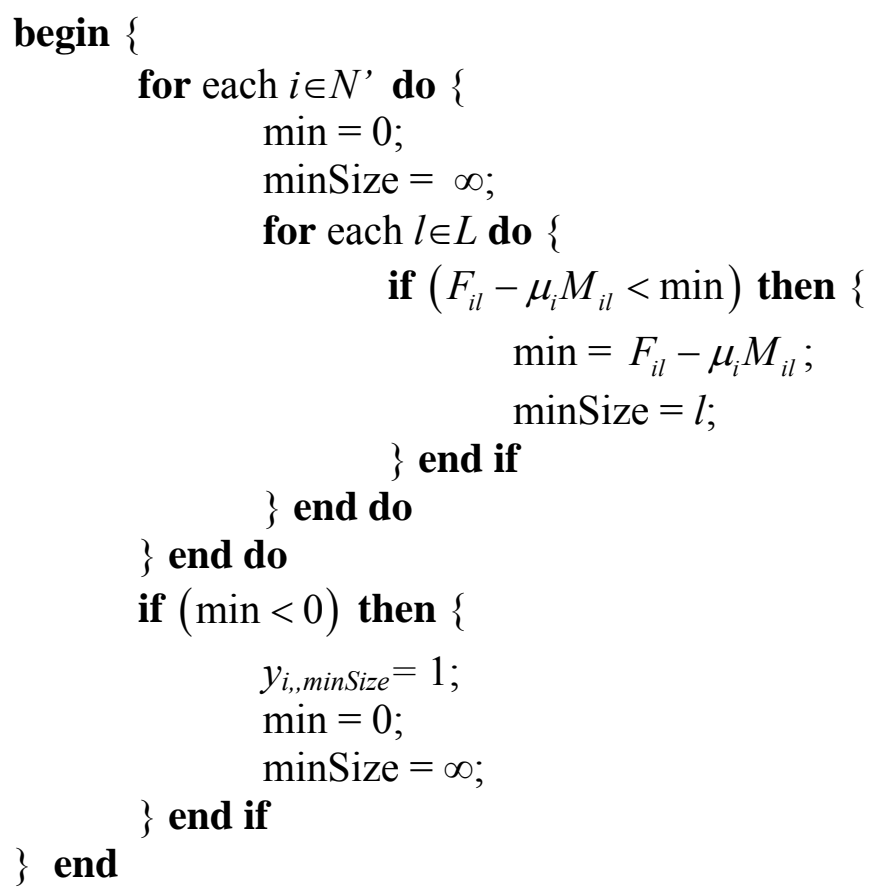

Figure 4-3: Greedy algorithm to determine the facility location and capacity

By the Lagrangian Bounding Principle, for any value of the Lagrangian multiplier $\mu$, the Lagrangian problem $L(\mu)$ constitutes a lower bound (in a minimization problem) on the optimal objective function value of the original problem (Ahuja et al., 1993). To obtain the lower bound value closest to the optimal solution, one must solve the Lagrangian multiplier problem $L^{*}=\max _{\mu} L(\mu)$. Following is the general procedure to solve the Lagrangian problem.

\section{$\underline{\text { General Procedure }}$}

Step0. Solve SP1 and SP2. Set $\left(y_{i l}, r_{i}^{k}, \theta\right)$ as the current optimal values. Set the lower bound equal to the objective values of the optimal solutions of SP1 and SP2:

$$
\text { lower } B=z_{S P 1}\left(y_{i l}\right)+z_{S P 2}\left(r_{i}^{k}, \theta\right)
$$

Step1. Check for feasibility. If 


$$
\sum_{k \in K} b_{i}^{k} r_{i}^{k} \leq \sum_{l \in L} M_{l} y_{i l}, \forall i \in N^{\prime}
$$

then stop, the solution is optimal. In the event that facilities with enough capacity to store the resource quantities determined by SP2 are open in the appropriate locations as a result of SP1, then the solution pertaining to the lower bound of the Lagrangian relaxation is feasible. Since the lower bound is the best solution that can be achieved, the algorithm has reached optimality. In the case where the facility locations and magnitude do not coincide with the supply allocation scheme, one must find feasible values for $y_{i l}$ based on the values of $r_{i}^{k}$ found in Step0. The objective value of this feasible solution added to the objective value of SP2 will be the upper bound.

$$
\text { upper } B=z\left(y_{i l}^{\text {feasible }}, r_{i}^{k}\right)+z_{S P 2}\left(r_{i}^{k}, \theta\right)
$$

Step2. Calculate error term:

$$
\varepsilon=\frac{\text { upper } B-\text { lower } B}{\text { upper } B}
$$

If $\varepsilon$ is less than a predetermined threshold value, then stop due to algorithm convergence.

Step3. Update Lagrangian multipliers using a sub-gradient method as described below. Then return to Step0. Subgradient optimization techniques are used to update the Lagrangian multipliers in the direction of change using a step size that ensures the algorithm convergence. If the subgradient is equal to 0 then the resource quantities use exactly the amount of storing space provided and the multipliers $\mu$ are kept at their current values. If the subgradient is negative then there is excess space to accommodate additional supplies and the Lagrangian multiplier $\mu$, which is like a toll for the resource, decreases. Else, the subgradient is positive, the warehouse capacity 
has been exceeded; less resource supplies must be purchased so the Lagrangian multipliers $\mu$ increase.

- First determine the gradient direction: subgradient $t_{i}=\sum_{k \in K} b_{i}^{k} r_{i}^{k}-\sum_{l \in L} M_{i l} y_{i l}$

- Second determine the step size - how far to move in the gradient direction:

$$
\text { step_size }=\frac{1}{\text { iterations }}
$$

- Finally update the multipliers: $\mu_{\text {iterations }+1}=\mu_{\text {iterations }}+($ step_size $) *\left(\right.$ subgradient $\left._{i}\right)$

\subsection{Lagrangian L-shaped Method Algorithm}

Figure 4-4 shows the structure of the resulting Lagrangian L-shaped method algorithm. The diagram shows how the L-shaped method is used as the master problem, the Lagrangian relaxation is used to solve the L-shaped method current problem and the network simplex algorithm solves the minimum cost flow problems finding the recourse function value.

\section{General procedure}

Step0. Set $\chi=\delta=0, z^{*}=\infty$, upper $B=\infty$, lower $B=0$. Set $\theta=-\infty$ and ignore during the initial computation.

Step1. Calculate error term: $\varepsilon=\frac{\text { upper } B-\text { lower } B}{\text { upperB } B}$.

If $\varepsilon$ is less than the predetermined threshold value then stop due to algorithm convergence and go to Step5, else go to Step2. 


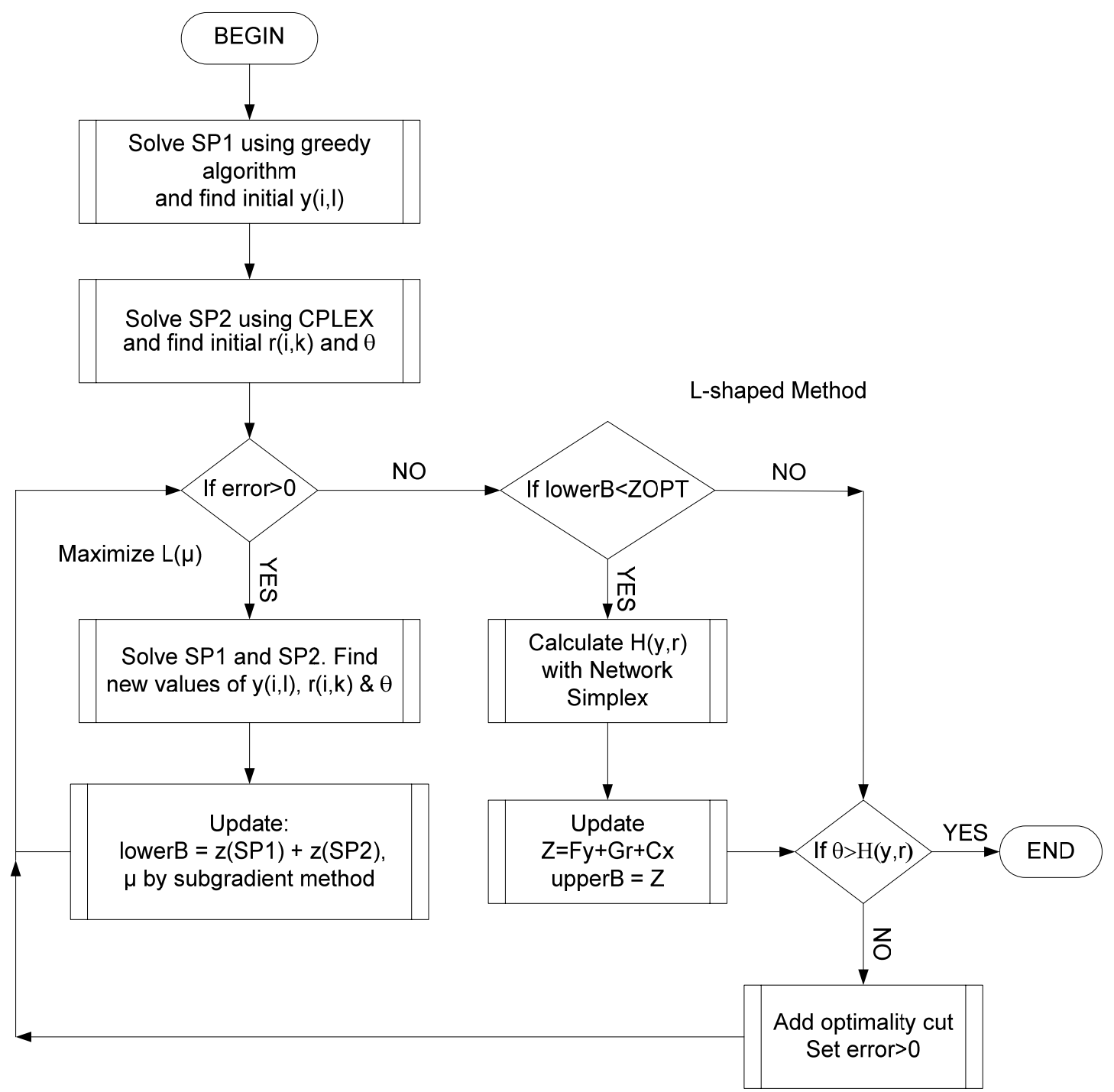

Figure 4-4: Lagrangian L-shaped method algorithm

Step2. Set $\delta=\delta+1$. Solve $\mathrm{SP} 1$ and $\mathrm{SP} 2$. Set $\left(y^{\delta}, r^{\delta}, \theta^{\delta}\right)$ as the current optimal solution. Set the lower bound equal to the objective values of the optimal solutions of SP1 and SP2:

$$
\text { lower } B=z_{S P 1}\left(y^{\delta}\right)+z_{S P 2}\left(r^{\delta}, \theta^{\delta}\right)
$$


Step3. Check for feasibility. Find values for $y_{i l}$ which provide a feasible solution based on the values of $r_{i}^{k}$ obtained from SP2:

$$
\sum_{k \in K} b_{i}^{k} r_{i}^{k} \leq \sum_{l \in L} M_{l} y_{i l}, \forall i \in N^{\prime} .
$$

The objective value of this feasible solution added to the objective value of SP2 will become the upper bound. upper $B=z\left(y_{i l}^{\text {feasible }}, r_{i}^{k}\right)+z_{S P 2}\left(r_{i}^{k}, \theta\right)$

Step4. Update Lagrangian multipliers using a sub-gradient method as described below. Then return to Step1.

- Determine the gradient direction: subgradient $t_{i}=\sum_{k \in K} b_{i}^{k} r_{i}^{k}-\sum_{l \in L} M_{i l} y_{i l}$

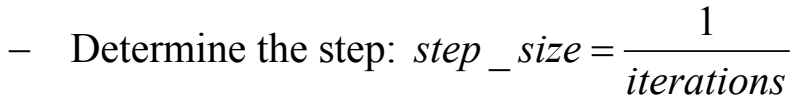

- Finally update the multipliers: $\mu_{\text {iterations }+1}=\mu_{\text {iterations }}+\left(\right.$ step_size $\left._{-}\right) *\left(\right.$ subgradient $\left._{i}\right)$

Note that "iterations" refer to the number of iterations of the Lagrangian relaxation solving the current problem.

Step5. Check the value of the current solution: if $\operatorname{lower} B\left(y^{\delta}, r^{\delta}, \theta^{\delta}\right)>z^{*}$ then compute $H\left(y^{\delta}, r^{\delta}\right)$ and $z^{\delta}=F^{T} y^{\delta}+q^{T} r^{\delta}+H\left(y^{\delta}, r^{\delta}\right)$. If $\quad z^{\delta}<z^{*} \quad$ then update $z^{*}=z^{\delta}$.

Step7. If $\theta^{\delta} \geq H\left(y^{\delta}, r^{\delta}\right)$ then fathom the current node (fathom by optimality cut) and end. Otherwise, impose one optimality cut, set $\chi=\chi+1$ and $\varepsilon=\infty$. Return to Step1. 


\section{CHAPTER 5. CASE STUDY}

\subsection{Case Study Problem}

A case study serves to illustrate the Stochastic Mixed Integer Program (SMIP) and to verify the Lagrangian L-shaped Method algorithm. In order to verify the methodology, the case study had is small enough to be solved in extensive form with a commercial software package, but detailed enough to paint a realistic picture. The problem focuses on hurricane threats in the Gulf of Mexico states. This region was chosen because of its high incidence of hurricane threats. The Atlantic Oceanographic and Meteorological Laboratory (AOML), a National Oceanic and Atmospheric Administration (NOAA) research facility, estimates that an average of 5.9 hurricanes strike the Atlantic Basin (East and Gulf states) each year, with an average of 2.2 being major hurricanes. The network created for the study is composed of 30 nodes and 58 links which include major cities of the Gulf coast states as shown in Figure 5-1 and Table 5-1.

In order to test the model and the algorithm, two sets of scenarios were developed, one of 21 and another of 51 scenarios. The scenarios are based on historical records of fifteen hurricane storms, ten major (categories 3 through 5) and five minor (categories 1 and 2). The descriptions of these two sets are presented in sections 5.2 and 5.3. The scenarios include both single storms and combinations of storms. A probability of occurrence is assigned to each scenario. These probabilities are based on approximately matching aggregate historical characteristics of hurricanes in the region, but should be treated as simply illustrative values. 
Table 5-1: Nodes included in the network

\begin{tabular}{|cl|cl|}
\hline Node & Description & Node & Description \\
\hline 1 & Brownsville & 16 & Birmingham \\
2 & Corpus Christi & 17 & Nashville \\
3 & San Antonio & 18 & Atlanta \\
4 & Dallas Ft. Worth & 19 & Columbia \\
5 & Houston & 20 & Charlotte \\
6 & Little Rock & 21 & Wilmington \\
7 & Memphis & 22 & Charleston \\
8 & Jackson & 23 & Savannah \\
9 & Monroe & 24 & Tallahassee \\
10 & Lake Charles & 25 & Int. I10 \& I75 \\
11 & Baton Rouge & 26 & Jacksonville \\
12 & Int. I10 \& I55 & 27 & Orlando \\
13 & New Orleans & 28 & Tampa \\
14 & Biloxi & 29 & Miami \\
15 & Mobile & 30 & Key West \\
\hline
\end{tabular}

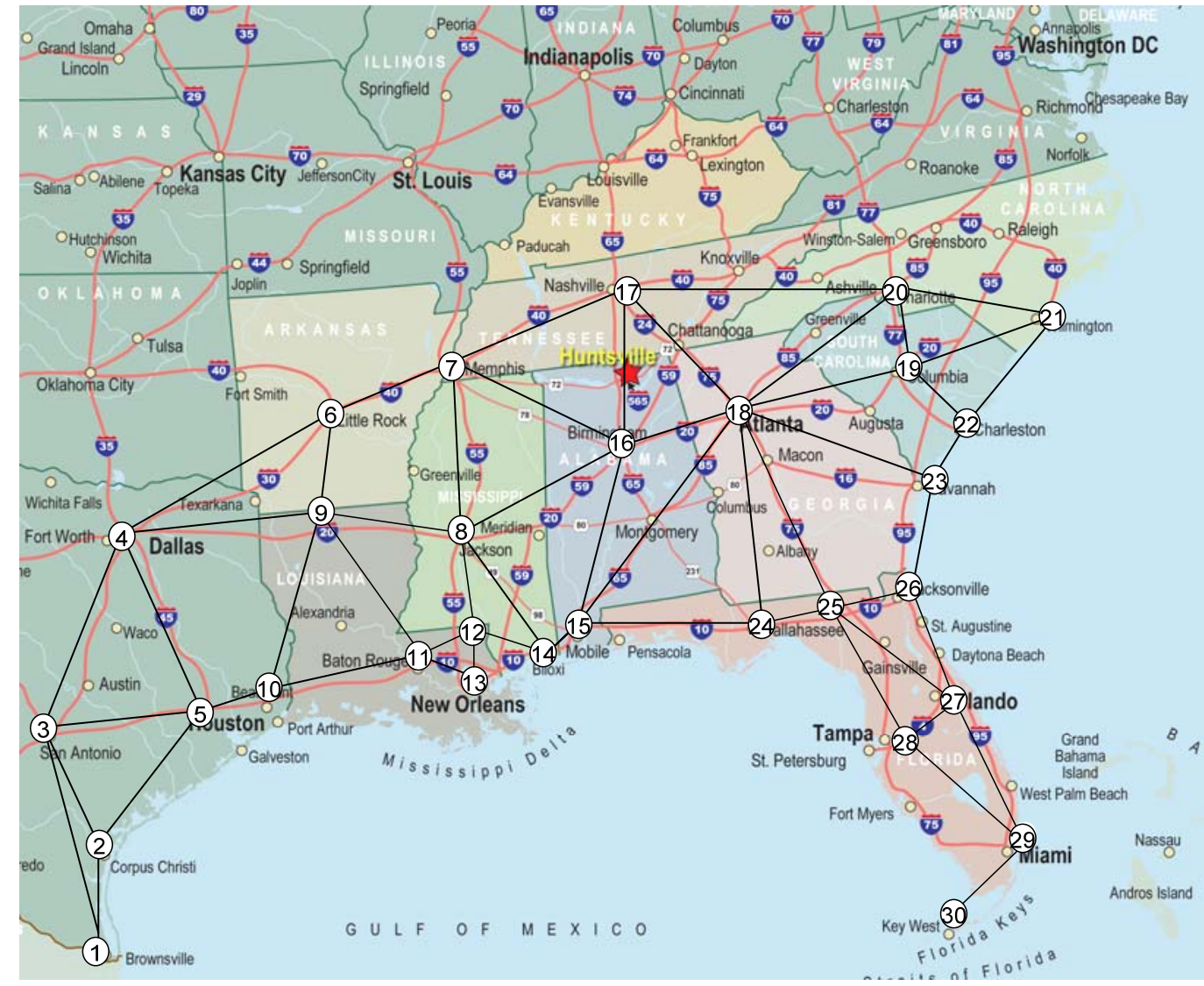

Figure 5-1: Case study network 


\subsection{Network costs and capacities}

The scenario definitions include damage to the transportation network, represented by reductions in the capacity of the nodes and the links in the network shown in Figure 5-1. The damage levels and locations are estimates, based on damage assessment reports (Beven, 2005; Post et al., 1990, 1993, 1994, 1999a, 1999b, 2000, 2003, 2005; US Army Corps, 1970, 1996, 1998; US Department of Commerce, 1997a, 1997b, 2006) provided by NOAA for the historical storms. Links can either be "damaged" (incurring a 50\% reduction in capacity) or "destroyed" (complete loss of capacity). The default values for the link capacities are set at 2000 units. The links and nodes affected are those located within the hurricanes' paths and the level of damage depended on the hurricane intensity at landfall. The facilities located at the hurricanes' landfall points are considered to be destroyed for major hurricanes and have reductions of capacity of $50 \%$ for minor hurricanes. In addition, any supplies prepositioned at the nodes in the network where hurricanes landfall are partially lost in the case of minor hurricanes and completely lost for major hurricanes. Table 5-2 summarizes the damage to the transportation infrastructure and potential storage facilities under the various storms included in the scenarios.

The link costs are estimates based on distance between the cities as shown in Figure 5-2 and the shipping costs of the specific commodities (trans) as described in section 5.4. However, the costs specified in Figure 5-2 have been rescaled for use in the case study, and do not reflect specific monetary units. 
Table 5-2: Roadway and facility damages resulting from hurricane passage

\begin{tabular}{|c|c|c|c|c|}
\hline \multirow{2}{*}{ Hurricane } & \multicolumn{2}{|c|}{ Roadway (links) } & \multicolumn{2}{|c|}{ Facility (nodes) } \\
\hline & Damaged & Destroyed & Damaged & Destroyed \\
\hline Alicia & -- & $(4,5) \mathrm{S}$ & -- & 5 \\
\hline Lili & $\begin{array}{c}(9,11) \\
(10,11) \\
(11,12) \\
(11,13) \\
(12,13) \\
(12,14)\end{array}$ & $(8,12)$ & 11 & -- \\
\hline Camille & -- & $\begin{array}{l}(12,14) \\
(14,15) \\
(15,24)\end{array}$ & -- & 14 \\
\hline Bonnie & -- & -- & 22 & -- \\
\hline Floyd & -- & $(17,20)$ & 22 & -- \\
\hline Andrew & $\begin{array}{l}(29,27) \mathrm{N} \\
(27,26) \mathrm{N} \\
(26,23) \mathrm{N}\end{array}$ & -- & 11 & 29 \\
\hline Opal & $\begin{array}{l}(15,16) \\
(15,24) \\
(15,18) \\
\end{array}$ & -- & -- & 15 \\
\hline Isabel & $\begin{array}{l}(20,21) \\
(19,21)\end{array}$ & $(21,22)$ & 21 & -- \\
\hline Katrina & $\begin{array}{c}(8,14) \\
(11,13) \\
(12,14)\end{array}$ & $(12,13)$ & 29 & 13 \\
\hline Bertha & $\begin{array}{l}(19,21) \\
(21,22)\end{array}$ & -- & -- & -- \\
\hline Fran & $\begin{array}{l}(19,21) \\
(22,23) \\
(23,26) \\
\end{array}$ & $(21,22)$ & -- & 21 \\
\hline Hugo & -- & -- & 22 & -- \\
\hline Emily & -- & -- & -- & -- \\
\hline Dennis & $(15,16)$ & $(15,24)$ & -- & -- \\
\hline Georges & $(29,30)$ & -- & 14 & 30 \\
\hline
\end{tabular}

$* \mathrm{~N}=$ northbound, $\mathrm{S}=$ southbound 


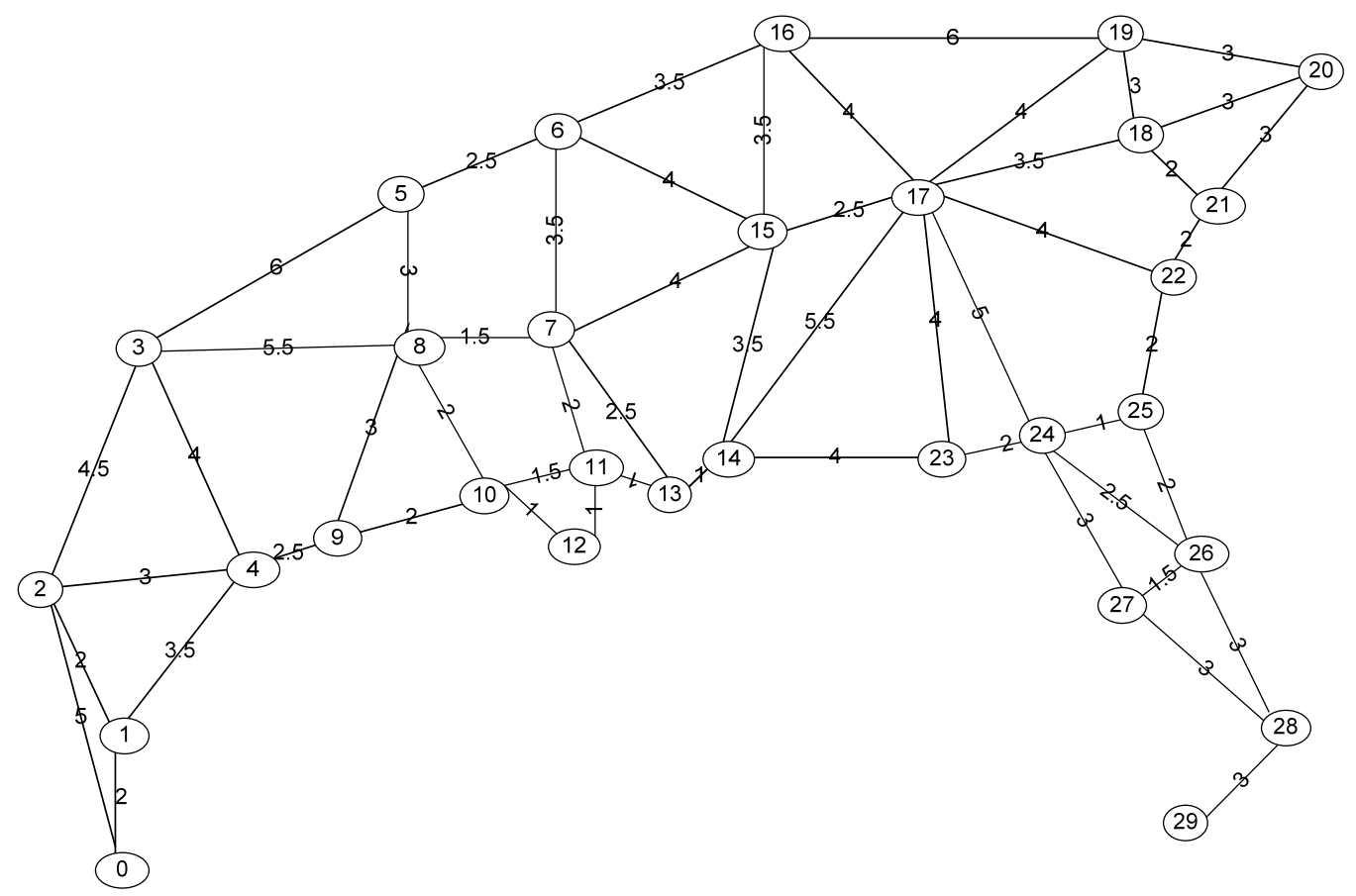

Figure 5-2: Link costs

\subsection{Demands}

Three emergency supplies are considered in the case study as shown in Figure 5-3. These are water, food and medical kits. Costs estimates were based on water stored in 10000 gallon-tanks, ready to eat meals (MREs) sold by the dozens and medical kits designed for emergencies. For the volume occupied by each commodity in cubic feet, its unit purchase price and unit transportation cost please refer to Table 5-6.

The demands for these commodities for each scenario are computed based on the number of evacuees and the total number of people seeking shelters in each of the hurricanes as recorded on the hurricane assessment reports. To estimate the emergency supplies demands shown in Table 5-3, the following assumptions were made: 

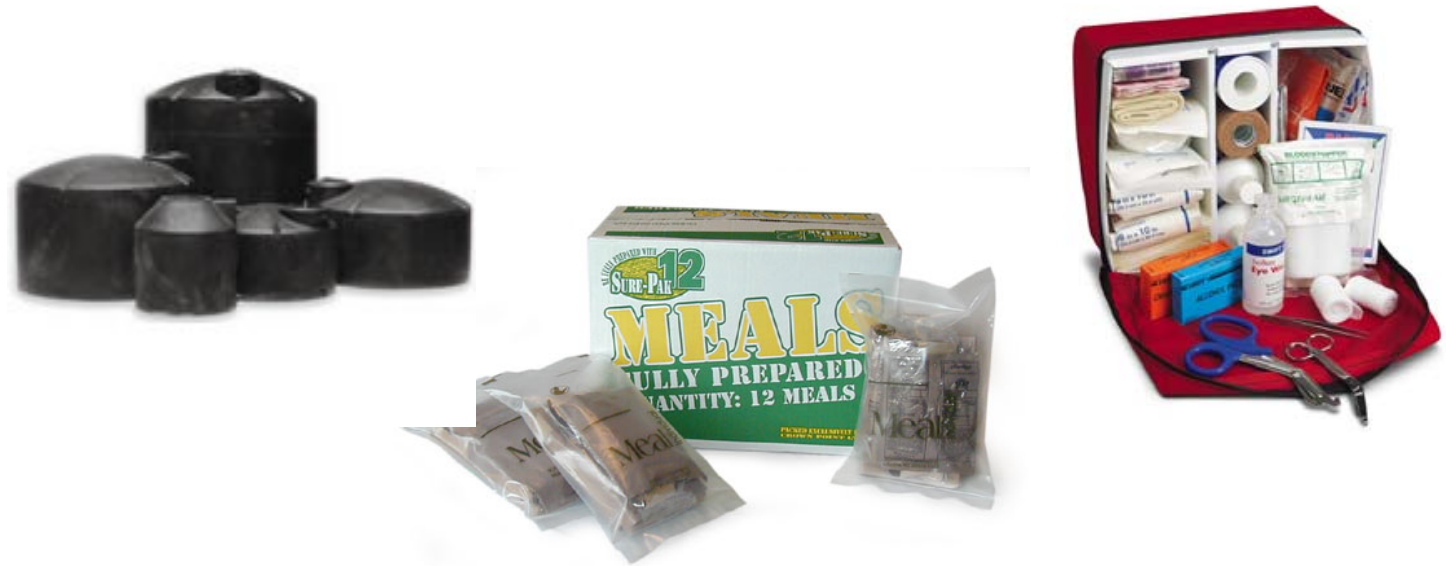

Figure 5-3: Commodities considered - water tanks, meals-ready-to-eat (MREs) and medical kits

- The expected demand for medical kits was calculated based on the number of people sheltered. Since the medical kit considered could provide service to four people, the estimated demand for medical kits $=$ number of people sheltered $/ 4$.

- An average person consumes three meals and two snacks per day. Similar to the expected number of medical kits, the demand for food was estimated based on the needs of the people staying in shelters. It was assumed that the average length of stay of people in a shelter would be 3 days for a minor hurricane and 7 days for a major hurricane. Thus the amount of food required during minor hurricanes $=$ $5 *$ sheltered $* 3$ and during major hurricanes $=5 *$ sheltered $* 7$.

- The water demand varies according to the number of evacuees. It was assumed that utilities take 3 days to recover from damages incurred during minor hurricanes and 10 days to recover from major hurricanes. Considering that the average person needs a gallon of water per day for food preparation and drinking according to the Department of Defense and the Office of Civil Defense (National Terror Alert Response Center website), the estimated water demand for minor hurricanes $=$ $1 *$ evacuees $* 3$ and the water demand for major hurricanes $=1 *$ evacuees $* 10$. 
Table 5-3: Emergency supply demands generated by hurricane threat

\begin{tabular}{|c|c|c|c|c|c|c|}
\hline Hurricane & Category & Sheltered & Evacuees & $\begin{array}{c}\text { Water } \\
(1000 \text { gallons })\end{array}$ & $\begin{array}{c}\text { Food } \\
(1000 \text { units })\end{array}$ & $\begin{array}{c}\text { Medicine } \\
\text { (units) }\end{array}$ \\
\hline Alicia & 3 & 25000 & 35000 & 350 & 525 & 500 \\
\hline Camille & 5 & 44152 & 56000 & 560 & 927 & 883 \\
\hline Bonnie & 2 & 20087 & 287000 & 861 & 181 & 402 \\
\hline Floyd & 2 & 188000 & 3000000 & 9000 & 1692 & 3760 \\
\hline Andrew & 4 & 84340 & 750000 & 7500 & 1771 & 1687 \\
\hline Opal & 3 & 87534 & 100000 & 1000 & 1838 & 1751 \\
\hline Isabel & 2 & 36000 & $200000^{*}$ & 600 & 324 & 720 \\
\hline Lili & 1 & 18000 & 500000 & 1500 & 162 & 360 \\
\hline Katrina & 5 & 3800000 & 1040000 & 10400 & 133000 & 950000 \\
\hline Bertha & 2 & 75000 & $750000^{*}$ & 2250 & 1125 & 18750 \\
\hline Fran & 3 & 50000 & $500000^{*}$ & 5000 & 1750 & 12500 \\
\hline Dennis & 3 & 18000 & $1800000^{*}$ & 18000 & 630 & 4500 \\
\hline Emily & 3 & 2282 & 281750 & 2818 & 80 & 571 \\
\hline Georges & 4 & 42204 & 223900 & 2239 & 1477 & 10551 \\
\hline Hugo & 4 & 112027 & 440000 & 4400 & 3921 & 28007 \\
\hline
\end{tabular}

* estimated: Number of sheltered is close to $10 \%$ number of evacuees

**estimated: Number of sheltered is close to $1 \%$ number of evacuees on tourist area

The demands per city per scenario were estimated based on their population densities and the intensity of the hurricane when reaching each city. The total demand calculated previously was distributed among the affected cities by calculating a fraction that compared these cities in terms of how much of its population might become victims of the storm. The estimate was done for each commodity and each city with the following equation:

$$
\operatorname{demand}_{\text {city,com modity }}^{\text {hurricane }}=\operatorname{demand}_{\text {com modity }}^{\text {hurricane }}\left(\frac{\text { Intensity }^{\text {hurricane }} * \text { Population }_{\text {city }}}{\sum_{\text {cities }} \text { Intensity }^{\text {hurricane }} * \text { Population }_{\text {city }}}\right)
$$


Table 5-4: Demand generated by the hurricanes used to construct the scenarios by commodity and city

\begin{tabular}{|c|c|c|c|c|c|}
\hline Hurricane & Node & City & $\begin{array}{c}\text { Water } \\
(1000 \text { gals })\end{array}$ & $\begin{array}{c}\text { Food } \\
(1000 \text { units })\end{array}$ & Medical Kits \\
\hline \multirow[t]{6}{*}{ Alicia } & 5 & Houston & 201 & 302 & 287 \\
\hline & 4 & Dallas & 40 & 60 & 57 \\
\hline & 3 & San Antonio & 80 & 121 & 115 \\
\hline & 2 & Corpus Christi & 19 & 28 & 27 \\
\hline & 1 & Brownsville & 5 & 7 & 7 \\
\hline & 10 & Lake Charles & 5 & 7 & 7 \\
\hline \multirow{7}{*}{ Camille } & 14 & Biloxi & 12 & 19 & 18 \\
\hline & 13 & New Orleans & 296 & 491 & 467 \\
\hline & 15 & Mobile & 44 & 73 & 69 \\
\hline & 8 & Jackson & 1 & 1 & 1 \\
\hline & 7 & Memphis & 52 & 86 & 82 \\
\hline & 17 & Nashville & 43 & 70 & 67 \\
\hline & 16 & Birmingham & 113 & 187 & 178 \\
\hline \multirow[t]{6}{*}{ Lili } & 10 & Lake Charles & 56 & 6 & 13 \\
\hline & 11 & Baton Rouge & 177 & 19 & 43 \\
\hline & 13 & New Orleans & 1014 & 110 & 243 \\
\hline & 9 & Monroe & 28 & 3 & 7 \\
\hline & 6 & Little Rock & 48 & 5 & 11 \\
\hline & 7 & Memphis & 177 & 19 & 43 \\
\hline \multirow[t]{4}{*}{ Bonnie } & 21 & Wilmington & 59 & 12 & 28 \\
\hline & 22 & Charleston & 314 & 66 & 146 \\
\hline & 20 & Charlotte & 309 & 65 & 144 \\
\hline & 19 & Columbia & 179 & 38 & 84 \\
\hline \multirow[t]{5}{*}{ Floyd } & 21 & Wilmington & 493 & 93 & 206 \\
\hline & 22 & Charleston & 2614 & 491 & 1092 \\
\hline & 20 & Charlotte & 2571 & 483 & 1074 \\
\hline & 19 & Columbia & 2986 & 561 & 1248 \\
\hline & 23 & Savannah & 335 & 63 & 140 \\
\hline \multirow[t]{8}{*}{ Andrew } & 29 & Miami & 3841 & 907 & 864 \\
\hline & 28 & Tampa & 1997 & 472 & 449 \\
\hline & 30 & Key West & 19 & 5 & 4 \\
\hline & 27 & Orlando & 819 & 194 & 184 \\
\hline & 13 & New Orleans & 666 & 157 & 150 \\
\hline & 11 & Baton Rouge & 116 & 27 & 26 \\
\hline & 10 & Lake Charles & 37 & 9 & 8 \\
\hline & $14,8,9$ & $\begin{array}{c}\text { Biloxi, Jackson, } \\
\text { Monroe }\end{array}$ & 1 & 0 & 0 \\
\hline \multirow[t]{5}{*}{ Opal } & 15 & Mobile & 121 & 222 & 212 \\
\hline & 14 & Biloxi & 21 & 39 & 37 \\
\hline & 24 & Tallahassee & 107 & 197 & 187 \\
\hline & 16 & Birmingham & 207 & 381 & 363 \\
\hline & 13 & New Orleans & 543 & 999 & 951 \\
\hline
\end{tabular}


Table 5-4 (Continued)

\begin{tabular}{|c|c|c|c|c|c|}
\hline \multirow[t]{4}{*}{ Bertha } & 21 & Wilmington & 111 & 56 & 928 \\
\hline & 22 & Charleston & 885 & 442 & 7373 \\
\hline & 20 & Charlotte & 580 & 290 & 4834 \\
\hline & 19 & Columbia & 674 & 337 & 5615 \\
\hline \multirow[t]{4}{*}{ Isabel } & 21 & Wilmington & 50 & 27 & 61 \\
\hline & 22 & Charleston & 134 & 72 & 160 \\
\hline & 20 & Charlotte & 263 & 142 & 316 \\
\hline & 19 & Columbia & 153 & 82 & 183 \\
\hline \multirow[t]{11}{*}{ Katrina } & 29 & Miami & 2945 & 37661 & 269009 \\
\hline & 28 & Tampa & 1531 & 19584 & 139885 \\
\hline & 30 & Key West & 15 & 188 & 1345 \\
\hline & 27 & Orlando & 942 & 12052 & 86083 \\
\hline & 13 & New Orleans & 3828 & 48960 & 349711 \\
\hline & 11 & Baton Rouge & 535 & 6839 & 48852 \\
\hline & 10 & Lake Charles & 127 & 1627 & 11621 \\
\hline & 14 & Biloxi & 15 & 193 & 1381 \\
\hline & 8 & Jackson & 3 & 41 & 292 \\
\hline & 9 & Monroe & 3 & 40 & 287 \\
\hline & 15 & Mobile & 455 & 5815 & 41535 \\
\hline \multirow[t]{5}{*}{ Fran } & 21 & Wilmington & 274 & 96 & 685 \\
\hline & 22 & Charleston & 1452 & 508 & 3631 \\
\hline & 20 & Charlotte & 1428 & 500 & 3571 \\
\hline & 19 & Columbia & 1659 & 581 & 4148 \\
\hline & 23 & Savannah & 186 & 65 & 466 \\
\hline \multirow[t]{5}{*}{ Hugo } & 22 & Charleston & 1732 & 1544 & 11027 \\
\hline & 21 & Wilmington & 109 & 97 & 694 \\
\hline & 19 & Columbia & 1484 & 1323 & 9448 \\
\hline & 20 & Charlotte & 852 & 759 & 5422 \\
\hline & 23 & Savannah & 222 & 198 & 1415 \\
\hline Emily & 21 & Wilmington & 2818 & 80 & 571 \\
\hline \multirow[t]{5}{*}{ Dennis } & 15 & Mobile & 9378 & 328 & 2344 \\
\hline & 24 & Tallahassee & 8293 & 290 & 2073 \\
\hline & 14 & Biloxi & 83 & 3 & 21 \\
\hline & 8 & Jackson & 44 & 2 & 11 \\
\hline & 30 & Key West & 202 & 7 & 51 \\
\hline \multirow[t]{5}{*}{ Georges } & 29 & Miami & 1513 & 998 & 7128 \\
\hline & 30 & Key West & 15 & 10 & 71 \\
\hline & 13 & New Orleans & 590 & 389 & 2780 \\
\hline & 14 & Biloxi & 5 & 3 & 22 \\
\hline & 15 & Mobile & 117 & 77 & 550 \\
\hline
\end{tabular}


Table 5-4 summarizes units of the various commodities required in specific cities for each of the fifteen historical storms. The units have been scaled for convenient representation (water demand is in thousands of gallons, for example). Demands for scenarios that include combinations of storms are the sum of individual storm demands.

\subsection{Resource and facilities unit costs and dimensions}

Table 5-5 contains the unit costs of opening a new facility (F) depending on its capacity. Table 5-6 contains the unit purchase prices (Q) of the resources by commodities, the unit shipments costs per unit of distance traveled (trans) and the volume (B) that each resource unit occupies.

Table 5-5: Costs of opening a facility depending on its storage capacity

\begin{tabular}{|c|c|c|c|}
\hline Size & Area $\left(\mathrm{ft}^{2}\right)$ & $\mathrm{F}(\$)$ & $\mathrm{M}\left(\mathrm{ft}^{3}\right)$ \\
\hline Small & 2800 & 19600 & 36400 \\
\hline Medium & 31400 & 188400 & 408200 \\
\hline Large & 60000 & 300000 & 780000 \\
\hline
\end{tabular}

Table 5-6: Unit purchase price, transportation costs and volume occupied by commodity

\begin{tabular}{|c|c|c|c|}
\hline Commodity & $\mathrm{Q}(\$)$ & $\mathrm{B}\left(\mathrm{ft}^{3}\right)$ & trans $(\$)$ \\
\hline Water (per 1000 gals) & 647.7 & 144.6 & 0.3 \\
\hline Food (per 1000 units) & 5420 & 83.33 & 0.04 \\
\hline Medicine & 140 & 1.16 & $5.80 \mathrm{E}-04$ \\
\hline
\end{tabular}

The unmet demand penalty costs are estimated as five times the resource purchase price, and the holding costs are estimated as one fourth the purchase price. Purchase prices for the three commodities are listed in Table 5-6. 


\subsection{Probability distribution}

The computation of the probabilities of the scenarios was divided into two stages. First determine the probability of occurrence of each hurricane and then calculate the probabilities of being hit by more than one hurricane within a small period of time. For the first stage a series of statements were developed based on historical records that established relationships between the probabilities of the hurricane threats. These are:

- Forty percent of all hurricanes that hit the U.S. hit the state of Florida.

- Based on the ratio of the average number of major (2.2) and minor (3.73) hurricanes that have hit the Atlantic Basin per year between the years 1965 and 2004 as shown in Appendix 1, it was assumed that a minor hurricane would have a higher likelihood of occurrence compared to a minor hurricane. The probability of a major hurricane would be $(2.2 / 3.73=0.6)$ the probability of a minor hurricane.

- It was assumed that the sum of the probabilities of all scenarios would be equal to 1.

- It was assumed that hurricanes of the same intensity that shared similar trajectories would have the same probability of occurrence and if their intensities were different, then the minor hurricane would have a larger likelihood of occurrence. A more detailed description of the probability calculations are included in sections 5.2 and 5.3 for the creation of the 21 and the 51 scenario test problems. 


\subsection{Twenty-one scenarios}

A subset of eight out of the fifteen hurricanes was included in the creation of the 21 scenarios. The storms included were major hurricanes Camille, Alicia, Andrew and Opal; and minor hurricanes Lili, Isabel, Floyd and Bonnie. The demands and the damages inflicted on the network due to these storms were as stated in Tables 5-2 and 5-4. Following are the statements used to determine the probability of each scenario.

\section{$\underline{\text { First stage }}$}

It was assumed that the probability of being hit by one hurricane was more likely than being attacked by two or non at all, so it was established that there should be a $75 \%$ chance of being hit by one hurricane and $25 \%$ of being hit by two or not being hit at all in a year. The sum of the probabilities of the eight single hurricanes threats would equal 0.75 .

Eq1: Bonnie + Floyd + Camille + Opal + Andrew + Isabel + Lili + Alicia $=0.75$

Since forty percent of all hurricanes that attack the United States hit Florida, it was established that the sum of the probabilities of the hurricanes that could affect Florida would be equal to 0.4 . Eq2: Bonnie + Floyd + Andrew + Opal $=0.4 *(0.75)$

Since hurricanes Alicia, Camille and Opal are major hurricanes with trajectories that affect the same region (i.e. the Gulf Coast); it was assumed that their probabilities would be the same. Eq3: Camille = Alicia $=$ Opal

Similarly, the probabilities of minor Cape Verde hurricanes Bonnie, Floyd and Isabel were assumed to be equal. Eq4: Bonnie $=$ Floyd $=$ Isabel 
Because hurricanes Alicia and Lili shared a similar trajectory, it was assumed that the probability of hurricane Alicia was 0.6 times the probability of hurricane Lili using the major/minor hurricane ratio. Eq5: Alicia $=0.6$ Lili

Using the major to minor hurricane ratio and assuming that the relationship will remain constant over all hurricane instances, the following statement was developed where the sum of the probabilities of all major hurricanes was equaled to the product of the sum of the probabilities of all minor hurricanes times the major/minor hurricane ratio:

Eq6: Alicia + Andrew + Opal + Camille $=0.6($ Bonnie + Floyd + Isabel + Lili $)$

Table 5-7 shows the results obtained after solving the six equations. Since the probability of occurrence of hurricane Andrew was negative, the value of each of the other hurricanes was truncated and the difference was used to increase the probability of hurricane Andrew resulting in the following values.

Table 5-7: Probability of occurrence of each hurricane

\begin{tabular}{|l|c|c|c|c|c|c|c|c|c|}
\hline Hurricanes & Alicia & Camille & Isabel & Andrew & Lili & Floyd & Bonnie & Opal & total \\
\hline $\begin{array}{c}\text { Probability }= \\
\text { Probabilities } \\
\text { rounded }=\end{array}$ & 0.0944 & 0.0944 & 0.1037 & -0.0020 & 0.1573 & 0.1038 & 0.1038 & 0.09448 & \\
\hline
\end{tabular}

\section{Second stage}

The hurricane threat combinations were selected depending on the hurricane intensity and trajectory. The goal was to create a series of scenarios that contained as many different hurricane damage combinations as possible, while keeping a smaller number of instances where both natural disasters affected the same region and a larger 
number of instances where the storms had different intensities. The resulting scenarios are shown in Table 5-8.

Table 5-8: Scenarios 9 through 21

\begin{tabular}{|c|c|c|c|c|}
\hline \multirow{2}{*}{ Scenarios } & \multicolumn{2}{|c|}{ Combinations } & \multicolumn{2}{c|}{ Regions affected } \\
\hline & Major & Minor & Same & Different \\
\hline Alicia + Camille & $\sqrt{ }$ & & $\sqrt{ }$ & \\
\hline Alicia + Floyd & $\sqrt{ }$ & $\sqrt{ }$ & & $\sqrt{ }$ \\
\hline Opal + Bonnie & $\sqrt{ }$ & $\sqrt{ }$ & & \\
\hline Opal + Andrew & $\sqrt{ }$ & & $\sqrt{ }$ & \\
\hline Lili + Andrew & $\sqrt{ }$ & $\sqrt{ }$ & & \\
\hline Lili + Isabel & & $\sqrt{ }$ & & $\sqrt{ }$ \\
\hline Alicia + Isabel & $\sqrt{ }$ & $\sqrt{ }$ & & $\sqrt{ }$ \\
\hline Opal + Isabel & $\sqrt{ }$ & $\sqrt{ }$ & & $\sqrt{ }$ \\
\hline Floyd + Camille & $\sqrt{ }$ & $\sqrt{ }$ & & $\sqrt{ }$ \\
\hline Camille + Andrew & $\sqrt{ }$ & & $\sqrt{ }$ & \\
\hline Camille + Opal & $\sqrt{ }$ & & $\sqrt{ }$ & \\
\hline Lili + Bonnie & & $\sqrt{ }$ & & $\sqrt{ }$ \\
\hline
\end{tabular}

It was assumed that each hurricane threat was an independent event. Therefore, the probability of two of these hurricanes developing within a small time frame would be the product of their probabilities. From the frequency distribution created with the number of hurricanes that have hit the Atlantic Basin between 1851 and 2004, it was determined that the probability of not having a hurricane threat in any given year was approximately 0.013 as shown in Table 5-9. The computations for the hurricane threat combinations and the resulting probability values are shown in Table 5-10. 
Table 5-9: Histogram of all hurricanes between 1851 and 2004

\begin{tabular}{|c|c|c|}
\hline Bin & Frequency & $\mathrm{P}=$ Frequency/Total \\
\hline 0 & 2 & 0.012987 \\
\hline 1 & 3 & 0.019481 \\
\hline 2 & 7 & 0.045455 \\
\hline 3 & 31 & 0.201299 \\
\hline 4 & 28 & 0.181818 \\
\hline 5 & 20 & 0.12987 \\
\hline 6 & 22 & 0.142857 \\
\hline 7 & 17 & 0.11039 \\
\hline 8 & 7 & 0.045455 \\
\hline 9 & 6 & 0.038961 \\
\hline More & 11 & 0.071429 \\
\hline Total $=$ & 154 & 1 \\
\hline
\end{tabular}

Table 5-10: Probabilities assigned to scenarios 9 through 21

\begin{tabular}{|c|c|c|c|c|c|}
\hline Scenarios & $\mathrm{P} 1$ & $\mathrm{P} 2$ & $\mathrm{P} 1 * \mathrm{P} 2$ & $(\mathrm{P} 1 * \mathrm{P} 2)+\mathrm{err} / \mathrm{s}$ & Rounded \\
\hline Alicia + Camille & 0.09 & 0.09 & 0.0081 & \begin{tabular}{|l|}
0.018629 \\
\end{tabular} & 0.018 \\
\hline Alicia + Floyd & 0.09 & 0.0975 & 0.008775 & 0.019304 & 0.019 \\
\hline Opal + Bonnie & 0.09 & 0.0975 & 0.008775 & 0.019304 & 0.019 \\
\hline Opal + Andrew & 0.09 & 0.0375 & 0.003375 & 0.013904 & 0.014 \\
\hline Lili + Andrew & 0.15 & 0.0375 & 0.005625 & 0.016154 & 0.016 \\
\hline Lili + Isabel & 0.15 & 0.0975 & 0.014625 & 0.025154 & 0.025 \\
\hline Alicia + Isabel & 0.09 & 0.0975 & 0.008775 & 0.019304 & 0.019 \\
\hline Opal + Isabel & 0.09 & 0.0975 & 0.008775 & 0.019304 & 0.019 \\
\hline Floyd + Camille & 0.0975 & 0.09 & 0.008775 & 0.019304 & 0.019 \\
\hline Camille + Andrew & 0.09 & 0.0375 & 0.003375 & 0.013904 & 0.014 \\
\hline Camille + Opal & 0.09 & 0.09 & 0.0081 & 0.018629 & 0.019 \\
\hline Lili + Bonnie & 0.15 & 0.0975 & 0.014625 & 0.025154 & 0.025 \\
\hline \multicolumn{3}{|l|}{ No hurricane threat } & 0.013 & 0.023529 & 0.024 \\
\hline \multirow{3}{*}{\multicolumn{2}{|c|}{ 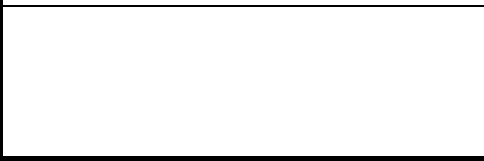 }} & total $=$ & 0.1147 & & 0.25 \\
\hline & & 0.25 -total $=$ & 0.1353 & & \\
\hline & & error $/ \mathrm{s}=$ & 0.010408 & & \\
\hline
\end{tabular}




\subsection{Fifty-one scenarios}

To test the performance of the SMIP, 51 scenarios were developed following the same logic as the one used to create the first 21 scenarios. In this instance the historical records of all 15 hurricanes (10 major, 5 minor) were included in the data set.

\section{First stage}

The relationships used in the first stage are as follows.

Since it was assumed that there was a higher probability of being threatened by one hurricane in any given year compared to being threatened by two or by none at all, for the first stage the probabilities of all single hurricane attacks were equaled to 0.75 . Eq1: Bonnie + Floyd + Camille + Opal + Andrew + Isabel + Lili + Alicia $=0.75$

Forty percent of all hurricanes that threaten the United States hit Florida. Eq2: Andrew + Opal + Katrina + Dennis + Georges $=0.4 *(0.75)$

Eq3 through Eq6 state the similarities between hurricanes based on their trajectories and strengths.

Eq3: Opal $=$ Dennis

Eq4: Katrina $=$ Andrew

Eq5: Fran $=$ Hugo $=$ Georges

Eq6: Bonnie $=$ Floyd $=$ Isabel

By the major to minor hurricane ratio, hurricanes that share the same trajectories but not their categories are included in the following relationships. 
Eq7: Camille $=0.6 *$ Lili

Eq8: Hugo $=0.6^{*}$ Bonnie

Assuming that the major to minor hurricane ratio still holds and assuming that the relationship remains constant over all hurricane instances, Eq9: Alicia + Andrew + Opal + Camille + Fran + Dennis + Emily + Georges + Hugo + Katrina $=0.6($ Bonnie + Floyd + Isabel + Lili + Bertha $)$.

These nine equations provide the hurricane probabilities included in Table 511. Since the probability of hurricane Alicia was negative, a constant fraction was transferred from each hurricane to Alicia. The latter is included in the adjusted probability column.

Table 5-11: Probability of occurrence of each of the 15 hurricanes

\begin{tabular}{|c|c|c|c|c|c|}
\hline Scenario & Hurricane & Category & Probability & $\begin{array}{c}\text { Adjusted } \\
\text { probability }\end{array}$ & Rounded \\
\hline 0 & Alicia & 3 & -0.3 & 0.023077 & 0.02308 \\
\hline 1 & Lili & 1 & 0.05388 & 0.030803 & 0.0308 \\
\hline 2 & Camille & 5 & 0.032328 & 0.009251 & 0.00925 \\
\hline 3 & Bonnie & 2 & 0.076705 & 0.053628 & 0.05363 \\
\hline 4 & Floyd & 2 & 0.076705 & 0.053628 & 0.05363 \\
\hline 5 & Andrew & 4 & 0.073082 & 0.050005 & 0.05 \\
\hline 6 & Opal & 3 & 0.053906 & 0.030829 & 0.03083 \\
\hline 7 & Isabel & 2 & 0.076705 & 0.053628 & 0.05363 \\
\hline 20 & Katrina & 5 & 0.073082 & 0.050005 & 0.05 \\
\hline 21 & Bertha & 2 & 0.184754 & 0.161677 & 0.16167 \\
\hline 22 & Fran & 3 & 0.046023 & 0.022946 & 0.02295 \\
\hline 23 & Hugo & 4 & 0.046023 & 0.022946 & 0.02295 \\
\hline 24 & Emily & 3 & 0.156876 & 0.133799 & 0.1338 \\
\hline 25 & Dennis & 3 & 0.053906 & 0.030829 & 0.03083 \\
\hline 26 & Georges & 4 & 0.046023 & 0.022946 & 0.02295 \\
\hline \multicolumn{7}{|c|}{ Total $=$} & & 0.75 & 0.75 & 0.75 \\
\hline
\end{tabular}


Table 5-12: Probability computations for the hurricane paired scenarios

\begin{tabular}{|c|c|c|c|c|c|c|c|}
\hline Scenario & Hurricane 1 & Hurricane2 & $P 1$ & $P 2$ & $P 1 * P 2$ & $(P 1 * P 2)+\frac{0.25-\text { total }}{36}$ & Rounded P \\
\hline 9 & Alicia & Camille & 0.02308 & 0.00925 & 0.000213 & 0.004671 & 0.0046 \\
\hline 10 & Alicia & Floyd & 0.02308 & 0.05363 & 0.001238 & 0.005695 & 0.0057 \\
\hline 11 & Opal & Bonnie & 0.03083 & 0.05363 & 0.001653 & 0.006111 & 0.0061 \\
\hline 12 & Opal & Andrew & 0.03083 & 0.05 & 0.001542 & 0.005999 & 0.006 \\
\hline 13 & Lili & Andrew & 0.0308 & 0.05 & 0.00154 & 0.005997 & 0.006 \\
\hline 14 & Lili & Isabel & 0.0308 & 0.05363 & 0.001652 & 0.006109 & 0.0061 \\
\hline 15 & Alicia & Isabel & 0.02308 & 0.05363 & 0.001238 & 0.005695 & 0.0057 \\
\hline 16 & Opal & Isabel & 0.03083 & 0.05363 & 0.001653 & 0.006111 & 0.0061 \\
\hline 17 & Floyd & Camille & 0.05363 & 0.00925 & 0.000496 & 0.004953 & 0.005 \\
\hline 18 & Camille & Andrew & 0.00925 & 0.05 & 0.000463 & 0.00492 & 0.005 \\
\hline 19 & Camille & Opal & 0.00925 & 0.03083 & 0.000285 & 0.004742 & 0.0047 \\
\hline 20 & Lili & Bonnie & \begin{tabular}{|l|}
0.0308 \\
\end{tabular} & 0.05363 & 0.001652 & 0.006109 & 0.0061 \\
\hline 28 & Katrina & Alicia & \begin{tabular}{|l|}
0.05 \\
\end{tabular} & 0.02308 & 0.001154 & 0.005611 & 0.0056 \\
\hline 29 & Bertha & Camille & \begin{tabular}{|l|}
0.16167 \\
\end{tabular} & 0.00925 & 0.001495 & 0.005953 & 0.006 \\
\hline 30 & Fran & Isabel & 0.02295 & 0.05363 & 0.001231 & 0.005688 & 0.0057 \\
\hline 31 & Hugo & Andrew & 0.02295 & 0.05 & 0.001148 & 0.005605 & 0.0056 \\
\hline 32 & Emily & Lili & \begin{tabular}{|l|}
0.1338 \\
\end{tabular} & 0.0308 & 0.004121 & 0.008578 & 0.0086 \\
\hline 33 & Dennis & Floyd & 0.03083 & 0.05363 & 0.001653 & 0.006111 & 0.0061 \\
\hline 34 & Georges & Bonnie & 0.02295 & 0.05363 & 0.001231 & 0.005688 & 0.0057 \\
\hline 35 & Georges & Opal & 0.02295 & 0.03083 & 0.000708 & 0.005165 & 0.0052 \\
\hline 36 & Dennis & Alicia & \begin{tabular}{|l|}
0.03083 \\
\end{tabular} & 0.02308 & 0.000712 & 0.005169 & 0.0052 \\
\hline 37 & Emily & Camille & \begin{tabular}{|l|}
0.1338 \\
\end{tabular} & 0.00925 & 0.001238 & 0.005695 & 0.0057 \\
\hline 38 & Hugo & Isabel & 0.02295 & 0.05363 & 0.001231 & 0.005688 & 0.0057 \\
\hline 39 & Fran & Andrew & \begin{tabular}{|l|}
0.02295 \\
\end{tabular} & 0.05 & 0.001148 & 0.005605 & 0.0056 \\
\hline 40 & Bertha & Lili & \begin{tabular}{|l|}
0.16167 \\
\end{tabular} & 0.0308 & 0.004979 & 0.009437 & 0.0094 \\
\hline 41 & Dennis & Camille & \begin{tabular}{|l|}
0.03083 \\
\end{tabular} & 0.00925 & 0.000285 & 0.004742 & 0.0047 \\
\hline 42 & Dennis & Bonnie & 0.03083 & 0.05363 & 0.001653 & 0.006111 & 0.0061 \\
\hline 43 & Dennis & Georges & \begin{tabular}{|l|}
0.03083 \\
\end{tabular} & 0.02295 & 0.000708 & 0.005165 & 0.0052 \\
\hline 44 & Bertha & Emily & \begin{tabular}{|l|}
0.16167 \\
\end{tabular} & 0.1338 & 0.021631 & 0.026089 & 0.0261 \\
\hline 45 & Bertha & Katrina & 0.16167 & 0.05 & 0.008084 & 0.012541 & 0.0125 \\
\hline 46 & Hugo & Georges & 0.02295 & 0.02295 & 0.000527 & 0.004984 & 0.005 \\
\hline 47 & Hugo & Emily & 0.02295 & 0.1338 & 0.003071 & 0.007528 & 0.0075 \\
\hline 48 & Katrina & Georges & \begin{tabular}{|l|}
0.05 \\
\end{tabular} & 0.02295 & 0.001148 & 0.005605 & 0.0056 \\
\hline 49 & Fran & Emily & 0.02295 & 0.1338 & 0.003071 & 0.007528 & 0.0075 \\
\hline 50 & Fran & Dennis & 0.02295 & 0.03083 & 0.000708 & 0.005165 & 0.0052 \\
\hline 51 & & & 0.012987 & 1 & 0.017444 & 0.0174 \\
\hline \multicolumn{4}{|c|}{ no hurricane } & total $=$ & 0.089544 & 0.25 & 0.25 \\
\hline
\end{tabular}




\section{$\underline{\text { Second stage }}$}

Table 5-12 contains the probabilities of all the scenarios with combined hurricane threats. Similar to the formation of the 21 scenarios, it was assumed that the probability of two hurricanes threatening the United States within a small time period was the product of their probabilities, where the combined probabilities were equaled to 0.25 as shown in Table $5-12$.

\subsection{Experiment Variations}

In order to test the computational efficiency of the algorithm, several variations of the case study problem were solved. These experiments were labeled as "bottleneck", "planes", and "variable demand". The bottleneck case restricts the feasibility region by limiting the amount of supplies that can be shipped through specific regions of the network. For the bottleneck, stronger link capacity restrictions were imposed on all arcs emanating from Atlanta; which is a centrally located highly populated city that can provide service to all the coastline states. Therefore, it is a perfect location for storing and distributing emergency response supplies. The link capacity for these arcs was decreased from 2000 units to 250 units.

In the case of the planes, these experiments include expensive but reliable alternatives of limited capacity of shipping resources from distant destinations regardless of the condition of the road segments. These alternatives are useful when there is extensive flooding and damages to the roadway network, like in the case of hurricane Katrina which isolated the victims of New Orleans from the neighboring towns; or when resources need to travel long distances in a small period of time. For the planes experiments the link capacity restrictions in the regions mostly affected by 
hurricane threats (i.e. Wilmington, Charleston, New Orleans, Baton Rouge, Biloxi, and Miami) were softened by the addition of the helicopter links as shown in Figure 54. These links had a capacity of 250 units and a cost of 50, which is much higher compared to the other links but comparable to the unmet demand penalty costs as shown in Figure 5-2.

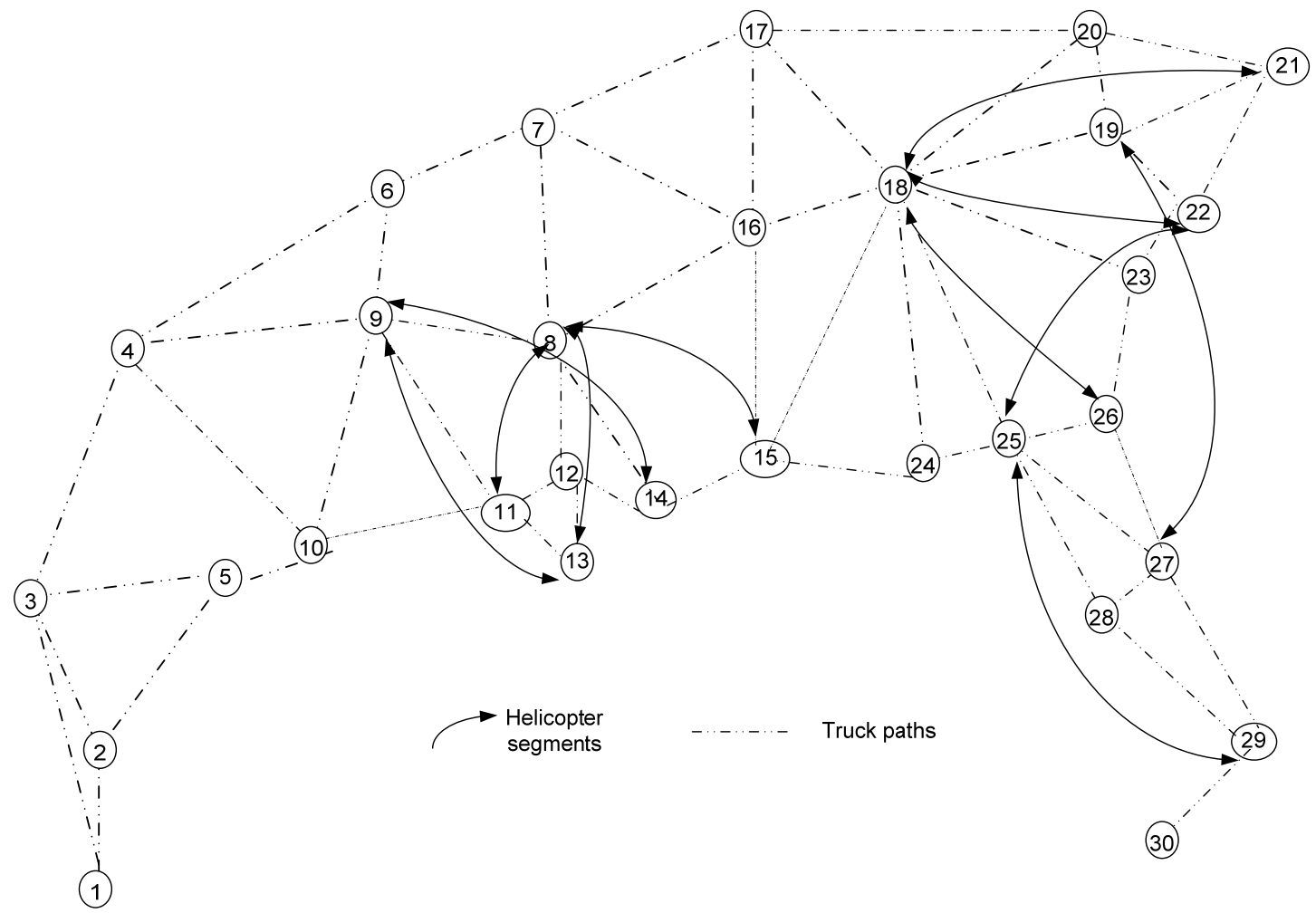

Figure 5-4: Plane links added to the network

The third experiment conducted was of varying the demand for the most damaged city of the worst case scenario. The latter is the city of New Orleans during hurricane Katrina. Due to the high level of damage and its population density, it generated one of the largest demand levels over all scenarios and cities. Due to the need of large quantities of resource, the algorithm would be forced to allocate supplies near New Orleans. However, if these high demand levels were relaxed, the feasible 
solution region would become less convex and more degenerate. This would increase the time needed to find an optimal solution and test the computational efficiency of the method. The results obtained from these experiments and the solution plan developed for the twenty-one and fifty-one scenarios case study are presented in Chapter 6. 


\section{CHAPTER 6. RESULTS}

This chapter contains the results obtained from solving the experiments described in section 5.8. These experiments are based on the stochastic mixed integer linear program formulation (presented in chapter 3) solved following the methodology of the Lagrangian L-shaped method as described in chapter 4. The solution includes

the location and capacity of the supplier facilities together with the quantities of various commodities stored in these facilities. For benchmarking, these experiments were also solved in extensive form using an integer linear program solver (i.e. Lingo). The results obtained from both sources are compared in the following sections. The results pertaining to the 21 -scenario case study are included in section 6.1 while the results pertaining to the 51-scenario case study are included in section 6.2 . Section 6.3 includes the analysis of the tolerance threshold selected for the Lagrangian L-shaped method (LLSM) and the Lagrangian Relaxation. Section 6.4 includes some concluding remarks.

\subsection{Twenty-one scenario solutions}

Table 6-1 shows the overall results obtained for the 21-scenario case (with unmet demand penalty costs equal to ten times the purchase price of the commodity) using the Lagrangian L-shaped method (LLSM) and the commercial software package Lingo. Table 6-1 includes information on the computational running times, the number of iterations and the optimal objective function values (z) achieved for the four experiments as discussed in section 5.8. 
For all the experiments shown in Table 6-1, the running times of the LLSM were a small fraction of the time required for Lingo. The LLSM running times ranged from $0.13 \%(13 / 10302)$ to $6.5 \%(16 / 247)$ of the Lingo running times. The objective values obtained from the LLSM heuristic are within approximately $1 \%$ of optimality (the exact solution obtained by Lingo).

Table 6-1: Results for 21-scenarios provided by the LLSM and Lingo

\begin{tabular}{|c|c|c|c|c|c|c|c|}
\hline \multicolumn{3}{|c|}{ summary } & \multicolumn{2}{c|}{ Lagrangian L-shaped Method } & \multicolumn{2}{c|}{ Lingo } \\
\hline case & planes & bottleneck & $\mathrm{z}$ & time (s) & iterations & time (s) & $\mathrm{Z}$ \\
\hline 1 & & & $2.45236 \mathrm{E}+07$ & 23 & 33 & 401 & $2.41933 \mathrm{E}+07$ \\
\hline 2 & $\sqrt{ }$ & $\sqrt{ }$ & $2.45909 \mathrm{E}+07$ & 16 & 26 & 247 & $2.42273 \mathrm{E}+07$ \\
\hline 3 & & $\sqrt{ }$ & $2.47882 \mathrm{E}+07$ & 13 & 23 & 10302 & $2.44633 \mathrm{E}+07$ \\
\hline 4 & $\sqrt{ }$ & & $2.46273 \mathrm{E}+07$ & 18 & 27 & 454 & $2.41933 \mathrm{E}+07$ \\
\hline
\end{tabular}

Table 6-2 contains the total number of facilities of each capacity and the total amount of pre-positioned resources allocated to the network for each case listed in Table 6-1, using the Lagrangian L-shaped method. As can be observed in Table 6-2 a total of four facilities were opened for cases 1 (no helicopter or bottleneck) and 2 (planes and bottleneck). Five facilities were opened for cases 3 (bottleneck), and 4 (planes). Table 6-2 also includes the first stage costs (i.e. the costs incurred by opening the facilities and purchasing the supplies). As can be observed in Table 6-2, the case with the lowest first stage cost and the highest overall objective value is case 3 (bottleneck). Case 3 is the most restricted due to the decrease in capacity of links emanating from Atlanta. This limited the amount of resource that could be shipped through certain links in the network, increasing the number of possible shipping paths and number of supplier facilities. On the other hand, the case with the most flexibility in transporting resources through the network is case 4 (with the added helicopter links). This permits the storing of more resources. Compared to case 1 which has the 
same demands and link capacity, case 4 was able to increase the amount of supplies in the network with smaller facilities, thus increasing the amount of met demand.

Table 6-2: First stage solutions obtained with the Lagrangian L-shaped method

\begin{tabular}{|c|c|c|c|c|c|c|c|}
\hline \multirow[b]{2}{*}{ case } & \multicolumn{3}{|c|}{ No. of facilities } & \multicolumn{3}{|c|}{ Resource purchased } & \multirow{2}{*}{$\begin{array}{c}\text { Facility and } \\
\text { supply costs }(\$)\end{array}$} \\
\hline & small & medium & large & $\begin{array}{c}\text { water } \\
\left(10^{3} \mathrm{gal}\right)\end{array}$ & $\begin{array}{l}\text { food } \\
\left(10^{3}\right)\end{array}$ & medicine & \\
\hline 1 & 1 & 1 & 2 & 8401 & 1905 & 2817 & $1.69714 \mathrm{E}+07$ \\
\hline 2 & 0 & 3 & 1 & 8866 & 1903 & 3420 & $1.73989 \mathrm{E}+07$ \\
\hline 3 & 2 & 2 & 1 & 8558 & 1880 & 3307 & $1.69087 \mathrm{E}+07$ \\
\hline 4 & 1 & 3 & 1 & 8699 & 2131 & 3181 & $1.85168 \mathrm{E}+07$ \\
\hline
\end{tabular}

Table 6-3: First stage solutions obtained with Lingo

\begin{tabular}{|c|c|c|c|c|c|c|c|}
\hline \multirow[b]{2}{*}{ case } & \multicolumn{3}{|c|}{ No. of facilities } & \multicolumn{3}{|c|}{ Resource purchased } & \multirow{2}{*}{$\begin{array}{c}\text { Facility and } \\
\text { supply costs }(\$)\end{array}$} \\
\hline & small & medium & large & $\begin{array}{c}\text { water } \\
\left(10^{3} \text { gal }\right)\end{array}$ & $\begin{array}{l}\text { food } \\
\left(10^{3}\right)\end{array}$ & medicine & \\
\hline 1 & 0 & 0 & 2 & 8998 & 2019 & 3435 & $1.78519 \mathrm{E}+07$ \\
\hline 2 & 0 & 0 & 2 & 8888 & 2019 & 3435 & $1.77805 \mathrm{E}+07$ \\
\hline 3 & 4 & 1 & 1 & 8000 & 2019 & 3435 & $1.71723 \mathrm{E}+07$ \\
\hline 4 & 0 & 0 & 2 & 8998 & 2019 & 3435 & $1.78519 \mathrm{E}+07$ \\
\hline
\end{tabular}

Table 6-3 contains the first stage solutions for the four cases as provided by integer solver Lingo. Compared to the results obtained with the LLSM shown in Table 6-2, the number of facilities is different but the total facility and supply costs are very similar. Table 6-3 shows the effects of the variations in the experiments in the quantities of water units purchased and for the case of the bottleneck (case 3), in the number of facilities available. The most restricted experiment is case 3 (bottleneck), where the links emanating from Atlanta had a decrease in capacity from 2000 units of each commodity to 250 units of each commodity. This decreased the total amount of water that could be transported, so the total amount of water purchased decreased from 8998 (thousand gallons of water for case 1) to 8000 (thousand gallons of water). The bottleneck effect also affected the number and location of the facilities. For cases 1, 2, 
and 4 two large facilities were opened (one in Columbia and another in Orlando). For case 3, six facilities were opened; four small (in New Orleans, Charlotte, Tallahassee, northern Florida), one medium facility in Orlando and one large in Columbia. Its inability to ship large quantities of supplies through the central links connecting Tennessee, Alabama, Georgia, North and South Carolina, caused the supplies to be divided among six facilities. The effect of the bottleneck was relaxed with the addition of helicopter links (case 2). In that case, the number of open facilities returned to two and there was a decrease of 110 thousand of gallons of water compared to the control case. Finally, no further improvements in the solution were reported for case 4 (planes only) indicating that the amount of supplies purchased in case 1 was not limited by the link capacities in the network.

Table 6-4: Maximum unmet demand level per experiment of the 21-scenario case study

\begin{tabular}{|c|c|c|c|c|c|c|}
\hline \multirow{2}{*}{ case } & \multicolumn{3}{|c|}{ LLSM } & \multicolumn{3}{c|}{ Lingo } \\
\cline { 2 - 7 } & water & food & medicine & water & food & medicine \\
\hline 1 & 1159 & 1704 & 1826 & 562 & 1590 & 1208 \\
\hline 2 & 694 & 1707 & 1223 & 672 & 1590 & 1208 \\
\hline 3 & 1002 & 1730 & 1336 & 1560 & 1590 & 1208 \\
\hline 4 & 861 & 1478 & 1462 & 562 & 1590 & 1208 \\
\hline
\end{tabular}

Table 6-4 contains the maximum level of unmet demand over all 21 scenarios for each of the experiments for the solutions obtained from LLSM and Lingo. The highest level of unmet demand for water and medicine occurs in case 1 (no planes and no bottleneck) and for food occurs in case 3 (bottleneck).

Table 6-5 contains the values for each term of the objective function for the solutions provided by both solvers in each of the four cases. For all but the last case, case 4 with additional plane links, the solutions given by LLSM had lower first stage and shipping costs incurring higher unmet demand penalty costs. For case 4 however, 
more water and food supplies were purchased in the LLSM solution, as shown in Table 6-4, thus incurring less unmet demand penalty costs.

Table 6-5: Objective value composition for the LLSM and the Lingo solutions

\begin{tabular}{|c|c|c|c|c|c|c|c|c|}
\hline \multirow[b]{2}{*}{ case } & \multicolumn{4}{|c|}{ LLSM solution values by objective term } & \multicolumn{4}{|c|}{ Lingo solution values by objective term } \\
\hline & \begin{tabular}{|c|}
$\begin{array}{c}\text { First stage } \\
\text { costs }\end{array}$ \\
\end{tabular} & \begin{tabular}{|c|}
$\begin{array}{c}\text { Shipment } \\
\text { costs }\end{array}$ \\
\end{tabular} & $\begin{array}{l}\text { Unmet demand } \\
\text { penalty costs }\end{array}$ & $\mathrm{Z}$ & $\begin{array}{c}\text { irst stage } \\
\text { costs }\end{array}$ & \begin{tabular}{|c|}
$\begin{array}{c}\text { Shipment } \\
\text { costs }\end{array}$ \\
\end{tabular} & $\begin{array}{c}\text { Unmet demand } \\
\text { penalty costs }\end{array}$ & $\mathrm{z}$ \\
\hline 1 & $1.697 \mathrm{E}+07$ & 2.302 & & $2.452 \mathrm{E}+07$ & $1.785 \mathrm{E}+07$ & 2.536 & & $2.419 \mathrm{E}+0$ \\
\hline 2 & $1.740 \mathrm{E}+07$ & $2.385 \mathrm{E}+06$ & +06 & $2.455 \mathrm{E}+07$ & $1.778 \mathrm{E}+07$ & $2.543 \mathrm{E}+06$ & & $2.423 \mathrm{E}$ \\
\hline 3 & $1.691 \mathrm{E}+07$ & $2.317 \mathrm{E}+06$ & $5.562 \mathrm{E}+06$ & $2.479 \mathrm{E}+07$ & $1.717 \mathrm{E}+07$ & 2.402 & $\mathrm{E}+06$ & $2.446 \mathrm{E}+07$ \\
\hline 4 & $1.852 \mathrm{E}+07$ & $2.630 \mathrm{E}+06$ & $3.481 \mathrm{E}+06$ & $2.463 \mathrm{E}+07$ & $1.785 \mathrm{E}+07$ & $2.536 \mathrm{E}+06$ & $3.805 \mathrm{E}+06$ & $2.419 \mathrm{E}+07$ \\
\hline
\end{tabular}

Figures 6-1 through 6-4 provide the pre-positioning strategies provided by the LLSM. In terms of the location of the facilities and the allocation of the supplies in the network, the resource pre-positioning strategies for the first three cases are very similar. Variations in the solutions are seen in case 4 due to the effects of the addition of the helicopter links, and in case 3 due to the decrease in link capacity for the arcs emanating from the Atlanta node. However, they all have one common property: since the shipment costs are low compared to the unmet demand penalty costs, for each of the scenarios the model will try to satisfy the demand regardless of the distance separating the supplier from the demand location. One can see for each of the following figures that the resources were stored in bundles. In other words, commodities are assigned to the facility, but these commodities are not divided among all the facilities. These bundles were generally located close to hurricane landfalls, areas prone to high demand levels. One must keep in mind that regardless of their initial location, these supplies are transferred to any demand location in the network whose total shipment cost is less than the unmet demand penalty cost.

Figure 6-1 shows the solution for the default case with no helicopter links or bottleneck (decrease in capacity for links emanating from Atlanta). The solution 
obtained by the LLSM for case 1 is to open two large facilities, one in Wilmington and another in Key West, one medium facility in Charlotte and a small in Tampa. Wilmington and Key West are strategically located near the landfall of many Cape Verde hurricanes so it is beneficial to open large facilities there. The large facilities are in charge of distributing the food supplies to the network, while the smallest facility distributes the commodity with the least volume (medical supplies). Water is supplied by the Charlotte facility in the northeast and the Key West facility in the southeast. All the facilities are located along the coastline which is a high hurricane incidence region.

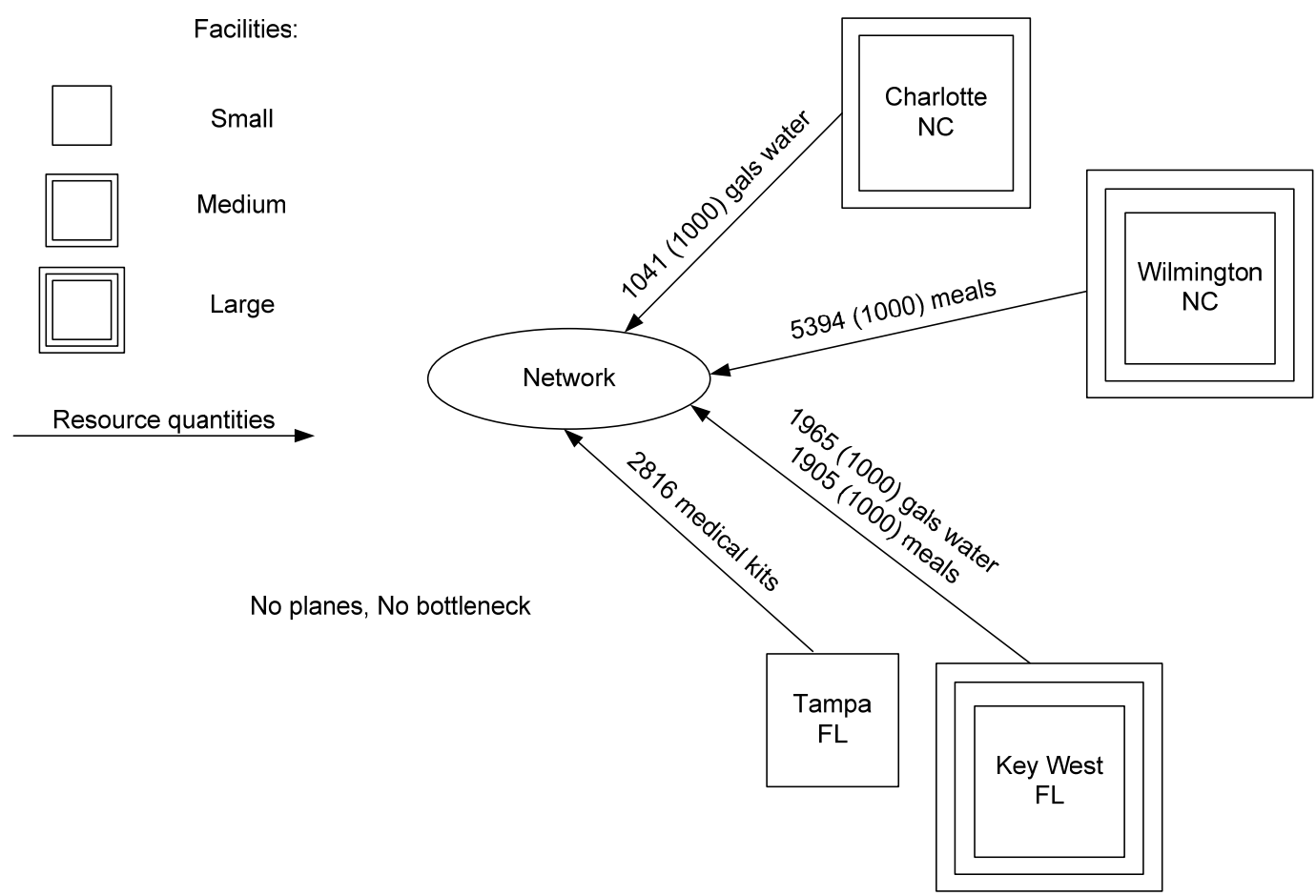

Figure 6-1: LLSM pre-positioning strategy case 1 (no planes, no bottleneck)

For the second case (planes and bottleneck) shown in Figure 6-2, one large and three medium facilities are opened. The effects caused by the bottleneck links emanating from Atlanta are shown in the new resource pre-positioning arrangement (compared to case 1 in Figure 6-1). The same locations were selected as in the 
previous case, however the sizes of the facilities in Florida changed. Instead of one small and one large facility in Florida, there are two medium facilities. This resulted from an exchange in commodity locations, in this case Charlotte will store medical kits and Tampa will store water.

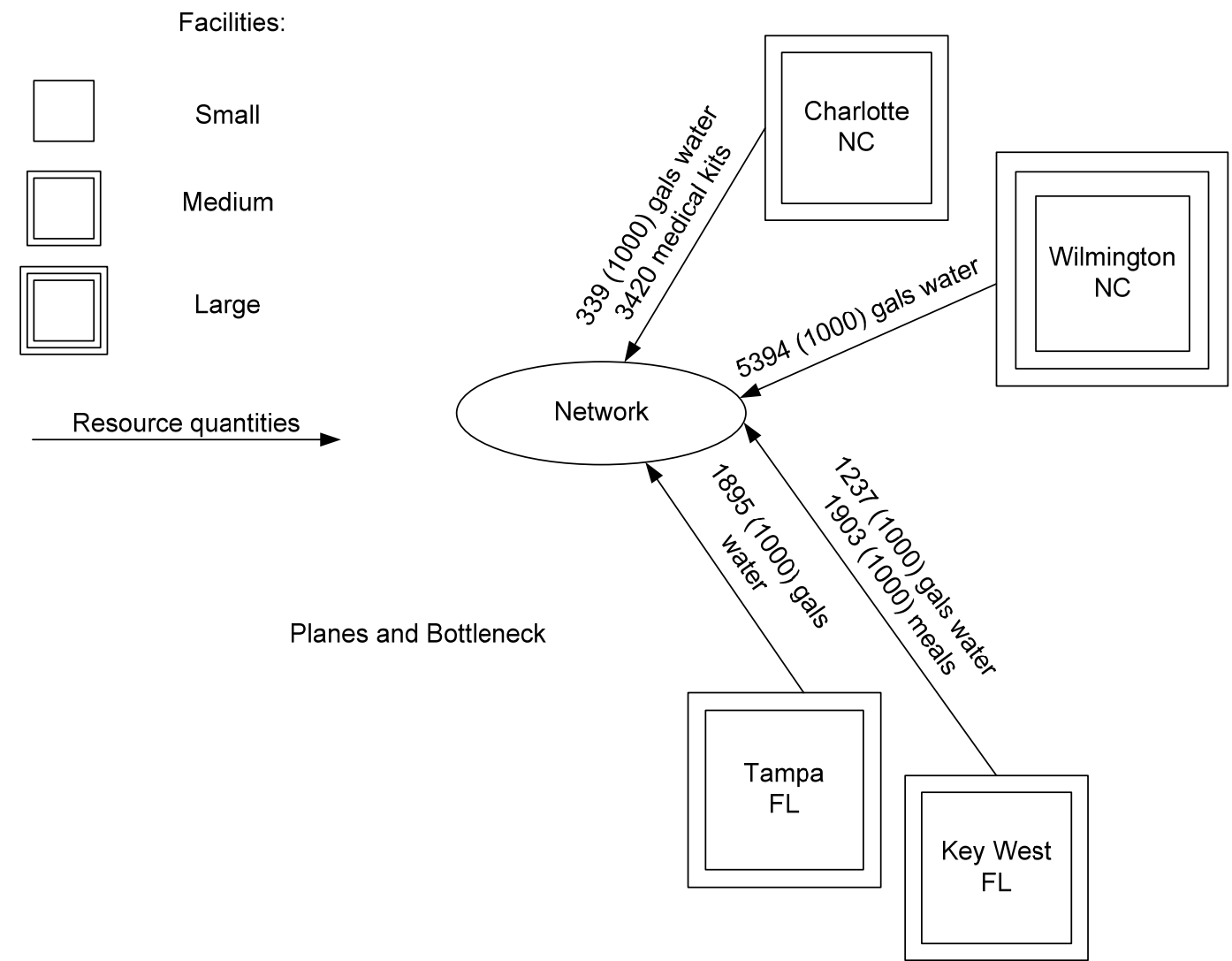

Figure 6-2: LLSM pre-positioning strategy case 2 (planes and bottleneck)

For the third case (bottleneck) shown in Figure 6-3, a total of five facilities (two small, two medium and one large) were opened. The largest facility was located in Wilmington as for the previous cases. The solution for the third case is similar to the previous two in terms of facility locations, with the exception of an added small facility located in Savannah. The latter is the result of the added link capacity restraint in the network of the links emanating from Atlanta. This small facility contains only 
enough water to satisfy local demands. As in case 2, Charlotte distributes the medical supplies and Key West the meals. The facilities located in Wilmington, Savannah, Tampa, and Key West; distribute the water to the demand points.

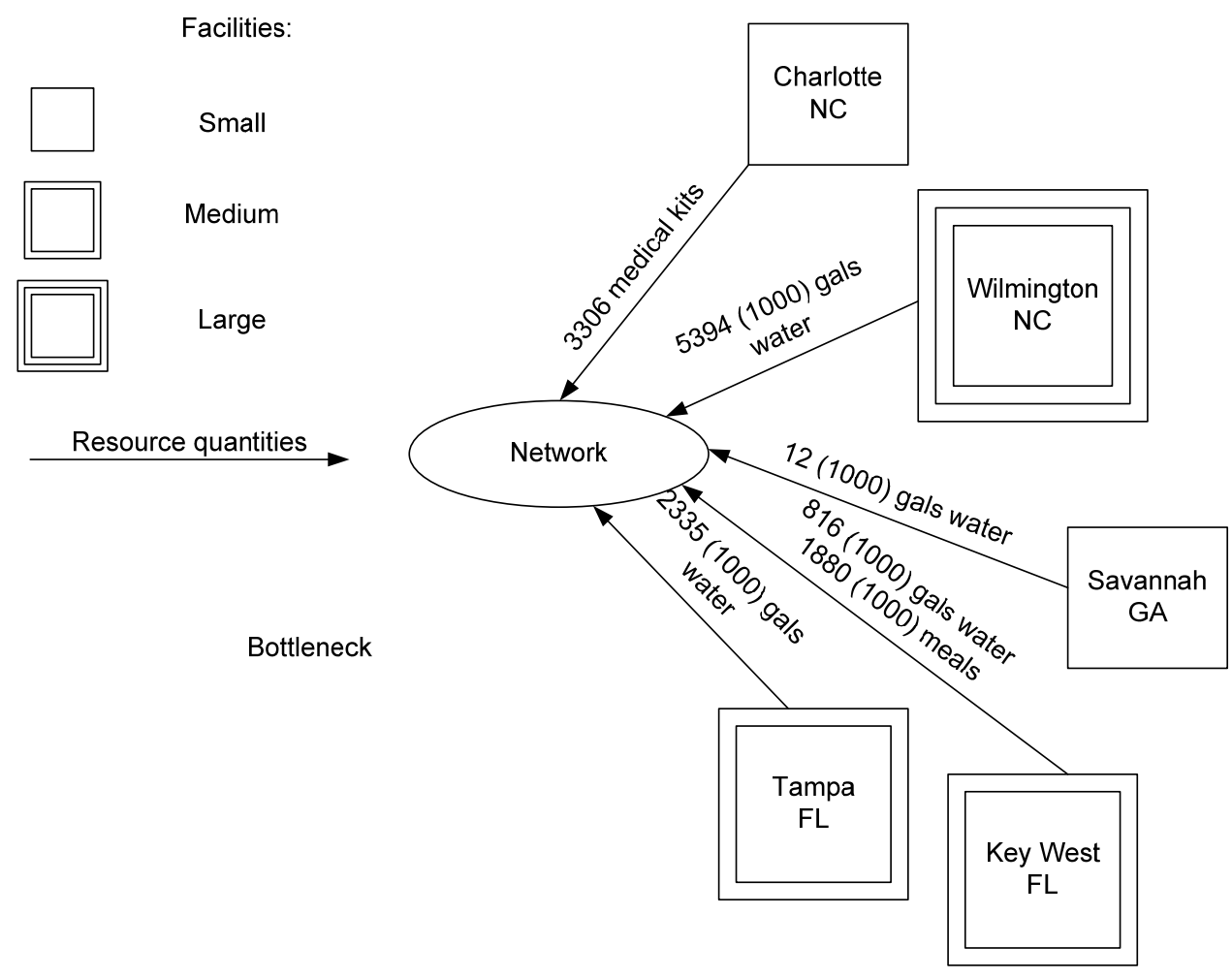

Figure 6-3: LLSM pre-positioning strategy for case 3 (bottleneck)

For case 4 (planes) shown in Figure 6-4, five facilities were opened (one small, three medium and one large). One of the five facilities is located in an airport node (Wilmington). The largest facility, located in Little Rock can provide service as easily to Gulf coast states as to the states affected by Cape Verde hurricanes while staying protected from storm damages. All the medical supplies were pre-positioned in Tampa, while the food supplies were divided amongst Wilmington and Key West. Four of the fives facilities are water distributors. 


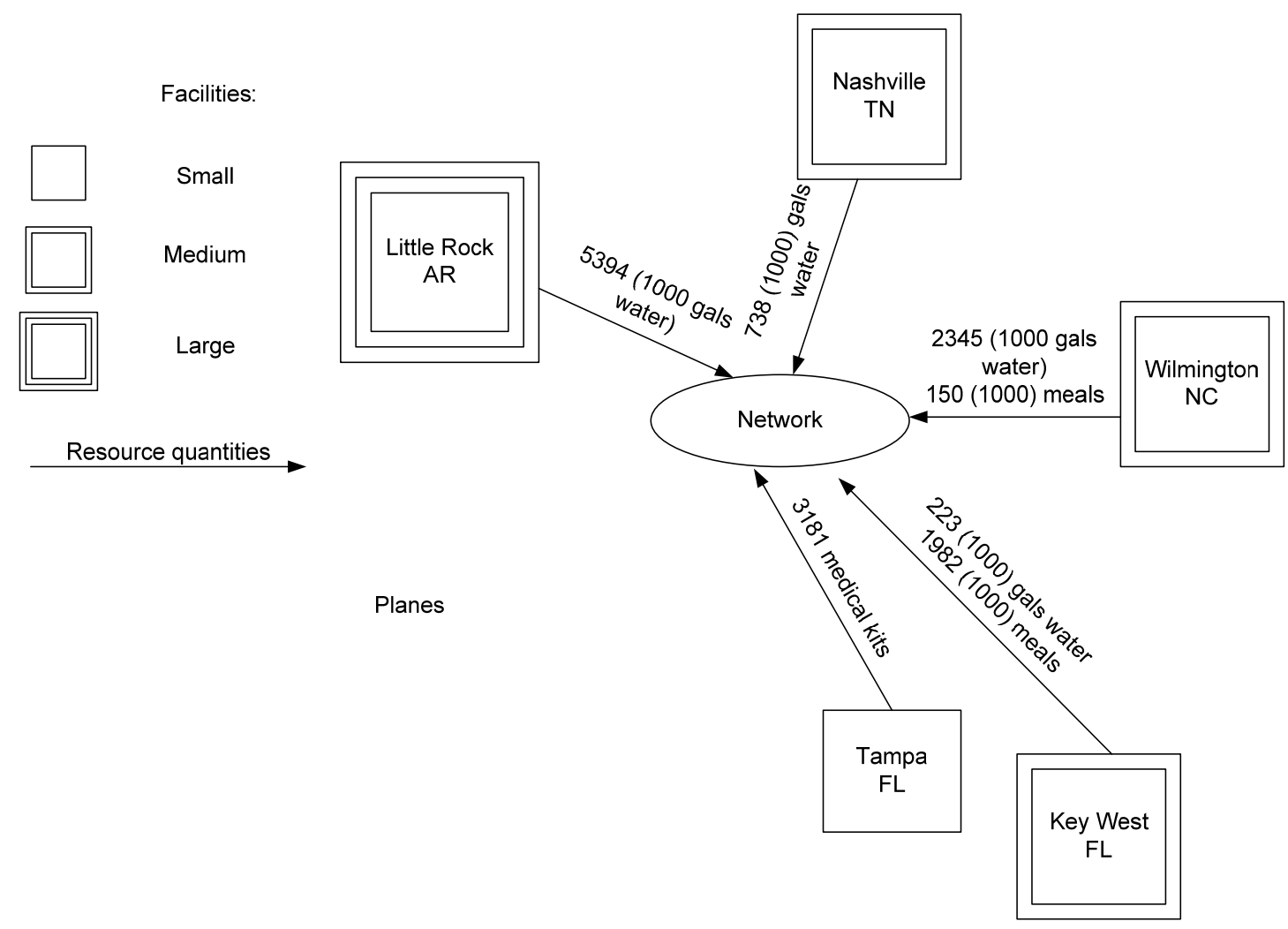

Figure 6-4: LLSM pre-positioning strategy for case 4 (planes)

These experiments were also solved with a reduction of unmet demand penalty costs of 50\%. Table 6-6 shows the first stage, second stage and total objective function values for each of the experiments. The experiment results show a transfer of cost between the first and the second stage, as the unmet demand penalty costs decrease. Even though the total objective values for the experiments with high unmet demand penalty costs were higher than the reduced cost versions, the second stage costs were lower. This result indicates that as the unmet demand penalty costs increase, more resources are allocated in order to satisfy the demand, as expected.

As shown in Table 6-6 the bottleneck, case 3, has the highest objective values and the highest first stage costs. The reason is that it is forced to open more facilities in order to meet the demand, due to the decrease in link capacities in the network. While only one facility is opened for the first two cases and two facilities are opened 
for the fourth case, three facilities are opened for case 3. Note that with the resource allocation suggested by the third solution, open one small facility in Brownsville (2903 medial kits), one medium facility in Dallas (1865 thousand meals), and one medium facility in Charlotte (2141 thousand gallons of water); the lowest second stage costs are achieved.

Table 6-6: Objective values per stage per experiment of the 21-scenario case study

\begin{tabular}{|c|c|c|c|c|c|c|}
\hline \multirow{2}{*}{ case } & \multicolumn{3}{|c|}{$50 \%$ unmet demand penalty costs } & \multicolumn{3}{c|}{$100 \%$ unmet demand penalty costs } \\
\cline { 2 - 8 } & total z & $1^{\text {st }}$ stage & $2^{\text {nd }}$ stage & total z & $1^{\text {st }}$ stage & $2^{\text {nd }}$ stage \\
\hline 1 & $2.066 \mathrm{E}+07$ & $1.120 \mathrm{E}+07$ & $9.457 \mathrm{E}+06$ & $2.452 \mathrm{E}+07$ & $1.697 \mathrm{E}+07$ & $7.552 \mathrm{E}+06$ \\
\hline 2 & $2.065 \mathrm{E}+07$ & $1.134 \mathrm{E}+07$ & $9.304 \mathrm{E}+06$ & $2.455 \mathrm{E}+07$ & $1.740 \mathrm{E}+07$ & $7.146 \mathrm{E}+06$ \\
\hline 3 & $2.083 \mathrm{E}+07$ & $1.230 \mathrm{E}+07$ & $8.532 \mathrm{E}+06$ & $2.479 \mathrm{E}+07$ & $1.691 \mathrm{E}+07$ & $7.879 \mathrm{E}+06$ \\
\hline 4 & $2.065 \mathrm{E}+07$ & $1.170 \mathrm{E}+07$ & $8.943 \mathrm{E}+06$ & $2.463 \mathrm{E}+07$ & $1.852 \mathrm{E}+07$ & $6.111 \mathrm{E}+06$ \\
\hline
\end{tabular}

\subsection{Fifty-one scenario solutions}

Following are the results obtained for the case study including 51 scenarios, as described in section 5.7. The first stage results obtained for the 51-scenario case study using the Lagrangian L-shaped method (LLSM) and the commercial software package Lingo are shown in Table 6-7. Table 6-7 also includes information on the computational running times, the number of iterations and the optimal objective function values $(\mathrm{z})$ achieved for the four experiments as discussed in section 5.8.

For all the experiments shown in Table 6-7, the running times of the LLSM were significantly lower than Lingo. The LLSM running times ranged from $0.22 \%$ (43/19955) to $10.0 \%(58 / 579)$ of the Lingo running times. Each of the eight cases was

solved in less than one minute using the LLSM, and the objective values obtained by the heuristic are within $0.05 \%$ of the optimal values. It is of some interest to note that 
in the most constrained case (case 3) for both 21 and 51 scenarios, Lingo requires a very long time (2.8 to 5.5 hours) to obtain a solution. However, this case creates no significant difficulty for the LLSM algorithm.

Table 6-7: Results for 51-scenarios provided by the LLSM and Lingo

\begin{tabular}{|c|c|c|c|c|c|c|c|}
\hline \multicolumn{2}{|c|}{ Summary } & \multicolumn{2}{c|}{ Lagrangian L-shaped Method } & \multicolumn{2}{c|}{ Lingo } \\
\hline case & planes & bottleneck & $\mathrm{z}$ & time (s) & iterations & time (s) & $\mathrm{z}$ \\
\hline 1 & & & $6.57516 \mathrm{E}+08$ & 59 & 54 & 926 & $6.57246 \mathrm{E}+08$ \\
\hline 2 & $\sqrt{ }$ & $\sqrt{ }$ & $6.58268 \mathrm{E}+08$ & 47 & 45 & 758 & $6.58401 \mathrm{E}+08$ \\
\hline 3 & & $\sqrt{ }$ & $6.59315 \mathrm{E}+08$ & 43 & 42 & 19955 & $6.59192 \mathrm{E}+08$ \\
\hline 4 & $\sqrt{ }$ & & $6.57252 \mathrm{E}+08$ & 58 & 53 & 579 & $6.56955 \mathrm{E}+08$ \\
\hline
\end{tabular}

Table 6-8 contains the total number of facilities of each capacity and the total amount of pre-positioned resources allocated to the network for each experiment of unmet demand penalty costs of ten times the purchase price as given by the Lagrangian L-shaped method. As can be observed in Table 6-8 a total of eight facilities were opened for case 1, with no helicopter or bottleneck links. Six facilities were opened in case 2 (planes and bottleneck), 3 (bottleneck), and 4 (planes). Table 68 also includes the first stage costs $($ i.e. the costs incurred by opening the facilities and purchasing the supplies). As can be observed in Tables 6-7 and 6-8, the case with the lowest overall objective value and lowest first stage costs is case 4 (the experiment that includes the additional helicopter links). For case 4, even though the number of facilities opened are fewer that the ones opened in case 1, more supplies were distributed throughout the network thanks to the increase in link capacity. Even though these added links had a limited capacity of only 250 units compared to the 2000 units of the default network links, and a higher link cost; using these links still decreased the total shipment costs incurred due to a decrease in unmet demand penalty costs. 
Table 6-8: First stage solutions obtained with the Lagrangian L-shaped method

\begin{tabular}{|c|c|c|c|c|c|c|c|}
\hline \multirow{2}{*}{ case } & \multicolumn{3}{|c|}{ No. of facilities } & \multicolumn{3}{c|}{ Resource purchased } & Facility and \\
\cline { 2 - 8 } & Small & Medium & Large & Water $\left(10^{3}\right.$ gal $)$ & Food $\left(10^{3}\right)$ & Medicine & $\begin{array}{c}\text { Supply } \\
\text { costs (\$) }\end{array}$ \\
\hline 1 & 4 & 3 & 1 & 8301 & 3643 & 21167 & $2.903 \mathrm{E}+07$ \\
\hline 2 & 1 & 3 & 2 & 7853 & 3541 & 21739 & $2.855 \mathrm{E}+07$ \\
\hline 3 & 2 & 3 & 1 & 7553 & 3668 & 23470 & $2.896 \mathrm{E}+07$ \\
\hline 4 & 2 & 3 & 1 & 8551 & 3327 & 19390 & $2.719 \mathrm{E}+07$ \\
\hline
\end{tabular}

The solutions obtained with Lingo, presented in Table 6-9, have certain similarities to those obtained in Table 6-8. The supply quantities for food and water and the total first stage costs are within 10\%. Generally (with the exception of case 3 ), the solutions obtained using Lingo have fewer open facilities. Once a facility is open, Lingo tends to allocate as much supply as possible, many times reaching the facility storage capacity. The LLSM creates the solutions a little differently. First it determines the amount of resource needed and the most strategic location for these supplies. It follows by determining the size of the facility that can store the allocated supplies. This method provides solutions that instead of filling open facilities to capacity; it will purchase only the supplies that are needed. This may result in solutions with open facilities with unused space. A solution with a closer proportion of available storage space to total occupied resource volume at each location can be achieved by providing the LLSM with a wider array of facility types (with varying respective capacities).

Table 6-9: First stage solutions obtained with Lingo

\begin{tabular}{|c|c|c|c|c|c|c|c|}
\hline \multirow[b]{2}{*}{ case } & \multicolumn{3}{|c|}{ No. of facilities } & \multicolumn{3}{|c|}{ Resource purchased } & \multirow{2}{*}{$\begin{array}{l}\text { Facility and } \\
\text { Supply costs } \\
(\$)\end{array}$} \\
\hline & small & medium & large & Water $\left(10^{3} \mathrm{gal}\right)$ & Food $\left(10^{3}\right)$ & medicine & \\
\hline 1 & 2 & 0 & 2 & 8999 & 3609 & 17258 & $2.844 \mathrm{E}+07$ \\
\hline 2 & 1 & 2 & 1 & 8303 & 3609 & 15615 & $2.782 \mathrm{E}+07$ \\
\hline 3 & 6 & 1 & 1 & 7526 & 3609 & 15115 & $2.716 \mathrm{E}+07$ \\
\hline 4 & 2 & 0 & 2 & 8999 & 3609 & 17508 & $2.848 \mathrm{E}+07$ \\
\hline
\end{tabular}


The solution for the 51-scenario case study differs from the 21-scenario case study. There is an increase in the values of first stage and the recourse objective terms as seen in Tables 6-7, 6-8 and 6-9. The latter is due to the high damage and demand levels included in the storms introduced in the sample of the 51-scenarios study. One of these is major hurricane Katrina which caused total devastation in New Orleans and the highest demand levels. In addition, the proportion of major to minor hurricanes increased from 1 to 1 for the 21 -scenario case study to 2 to 1 for the 51 -scenario case study. Therefore, there is an increase in the expected demand and the damage levels in the network.

Table 6-10 contains the maximum level of unmet demand over all 51 scenarios for each of the experiments for the solutions obtained from LLSM and Lingo. Compared to the results presented in Table 6-5 for the 21-scenario case, the maximum level of unmet demand for any scenario increased by one, two and three orders of magnitude for water, food and medicine, respectively. This difference is the result of high demand levels occurring in certain scenarios that have low incidence probabilities. These scenarios include hurricane threats from rare and devastating storms such as Dennis, Katrina, and Emily. Because of their low probability of occurrence, the solution will allocate less resource than the one needed for such extreme instances. One can see from Table 6-9 that both solvers reached solutions that left approximately the same maximum levels of unmet demand.

Table 6-10: Maximum unmet demand level per experiment of the 51-scenarios case study

\begin{tabular}{|c|c|c|c|c|c|c|}
\hline \multirow{2}{*}{ case } & \multicolumn{3}{|c|}{ LLSM } & \multicolumn{3}{c|}{ Lingo } \\
\cline { 2 - 7 } & water & food & medicine & water & food & medicine \\
\hline 1 & 18699 & 130834 & 947583 & 18001 & 130868 & 951492 \\
\hline 2 & 19147 & 130936 & 959591 & 18697 & 130868 & 953135 \\
\hline 3 & 19447 & 130809 & 945280 & 19474 & 130868 & 953635 \\
\hline 4 & 18449 & 131150 & 949360 & 18001 & 130868 & 951242 \\
\hline
\end{tabular}


Tables 6-11 through 6-14 show the results for the experiments as described in section 5.8 of the data set for the 51-scenario case study. The first, Table 6-11 shows the solution for the default case with no bottlenecks or helicopter links. The model allocated all the food in a medium facility in Texas, location that is not affected by any hurricane trajectory. Most of the water is stored in the large facility in Charlotte, location that is close to regions with high hurricane threats. Three facilities were located in Florida, two in South Carolina and one in North Carolina, which are areas of high hurricane activity. However, only two facilities, the one in Charleston and the one in Miami, suffer damages in a few scenarios.

Table 6-11: Solution for case 1 (no planes, no bottleneck)

\begin{tabular}{|c|c|c|c|c|}
\hline City & Facility size & $\begin{array}{c}\text { Water } \\
\text { (thousand gals) }\end{array}$ & $\begin{array}{c}\text { Food } \\
\text { (thousand meals) }\end{array}$ & Medical kits \\
\hline Brownsville, TX & Medium & 0 & 3643 & 0 \\
\hline Mobile, AL & Small & 0 & 0 & 4351 \\
\hline Columbia, SC & Small & 109 & 0 & 4792 \\
\hline Charlotte, NC & Large & 5362 & 0 & 3983 \\
\hline Charleston, SC & Small & 0 & 0 & 6688 \\
\hline Tallahassee, FL & Medium & 1489 & 0 & 0 \\
\hline Tampa, FL & Medium & 1341 & 0 & 0 \\
\hline Miami, FL & Small & 0 & 0 & 1353 \\
\hline \multicolumn{2}{|r|}{ Total $=$} & 8301 & 3643 & 21167 \\
\hline
\end{tabular}

Table 6-12 contains the solution for case 2 which includes planes and bottleneck links. Two of the chosen locations have helicopter airports: Columbia and Orlando. The largest facilities are located in Tallahassee and Columbia. Most of the water was distributed among these two large facilities. The remaining water was stored in Orlando. The food supplies were divided among the facility in Nashville and the one in Tampa, while the medical supplies are in Brownsville and Columbia. The bottleneck effect is seen in the selection of Columbia, one of the nearest neighbors of Atlanta, as a major supplier instead of the aforementioned. Fewer supplies were 
allocated for case 2 than for case 1 . Note that even though case has added link capacity of the helicopter links, the decrease in capacity caused by the bottleneck decreased the amount of supplies.

Table 6-12: Solution for case 2 (planes and bottleneck)

\begin{tabular}{|c|c|c|c|c|}
\hline City & Facility size & $\begin{array}{c}\text { Water } \\
\text { (thousand gals) }\end{array}$ & $\begin{array}{c}\text { Food } \\
\text { (thousand meals) }\end{array}$ & Medical kits \\
\hline Brownsville, TX & Small & 0 & 0 & 3573 \\
\hline Nashville, TN & Medium & 0 & 2427 & 0 \\
\hline Columbia, SC & Large & 3523 & 0 & 5586 \\
\hline Tallahassee, FL & Large & 2941 & 0 & 0 \\
\hline Orlando, FL & Medium & 1389 & 0 & 0 \\
\hline Tampa, FL & Medium & 0 & 1114 & 0 \\
\hline \multicolumn{2}{|c|}{ Total $=$} & 7853 & 3541 & 9159 \\
\hline
\end{tabular}

The solution for case 3, bottleneck only, is presented in Table 6-13. Similar to case 2, a large facility was opened in Columbia. In this case, three facilities were opened around Atlanta (e.g. Columbia, Charleston, and Tallahassee) and most of the water and medical supplies for the system were distributed among them. In this case, the resources were located mostly along the east coastline, which has higher hurricane incidence. Two facilities were opened in Louisiana with part of the food and medical kits, while all the water was stored in the eastern states.

Table 6-13: Solution for case 3 (bottleneck)

\begin{tabular}{|c|c|c|c|c|}
\hline City & Facility size & $\begin{array}{c}\text { Water } \\
\text { (thousand gals) }\end{array}$ & $\begin{array}{c}\text { Food } \\
\text { (thousand meals) }\end{array}$ & Medical kits \\
\hline Lake Charles, LA & Small & 0 & 0 & 5619 \\
\hline N. New Orleans, LA & Medium & 0 & 2106 & 0 \\
\hline Columbia, SC & Large & 5327 & 0 & 8379 \\
\hline Charleston, SC & Small & 162 & 0 & 9473 \\
\hline Tallahassee, FL & Medium & 576 & 1562 & 0 \\
\hline Tampa, FL & Medium & 1488 & 0 & 0 \\
\hline \multicolumn{2}{|c|}{} & 7553 & 3668 & 23470 \\
\hline
\end{tabular}


Table 6-14 has the solution for case 4, which includes the additional helicopter links. Three of the facilities are located in airport nodes (e.g. Charleston, Orlando and Miami). Two of these facilities (Charleston and Miami) suffer reductions in capacity and thus in resource quantities for certain scenarios. These reductions in capacity and in resource quantities, forces the model to purchase larger quantities of supplies than can be used in certain cases. In this case, both facilities contain medical supplies. The model would rather incur the costs for loss of supplies than for unmet demand. Similar to the second case (Table 6-12), three facilities are opened in Florida and one in Texas. The largest facility is located in Charlotte, a location that can send supplies to all hurricane landfall points on record. The solution for case 4 is very similar to the solution for case 2 (planes and bottleneck) in terms of facility locations, but the latter has a higher number of facilities due to the effects of the bottleneck links.

Table 6-14: Solution for case 4 (planes)

\begin{tabular}{|c|c|c|c|c|}
\hline City & Facility size & $\begin{array}{c}\text { Water } \\
\text { (thousand gals) }\end{array}$ & $\begin{array}{c}\text { Food } \\
\text { (thousand meals) }\end{array}$ & Medical kits \\
\hline Brownsville, TX & Medium & 0 & 3327 & 0 \\
\hline Charlotte, NC & Large & 4282 & 0 & 9987 \\
\hline Tallahassee, FL & Medium & 2278 & 0 & 0 \\
\hline Orlando, FL & Medium & 1991 & 0 & 0 \\
\hline Miami, FL & Small & 0 & 0 & 4489 \\
\hline Charleston, SC & Small & 0 & 0 & 4914 \\
\hline \multicolumn{2}{|c|}{ Total $=$} & 8551 & 3327 & 19390 \\
\hline
\end{tabular}

The highest demand levels recorded among the storms included in the 51scenario case study were caused by hurricane Katrina. Among the cities affected, the most damage and the highest demand levels were produced in the city of New Orleans. Conducting the following experiments, we found that changes in the data affect the shape of the objective function, increasing the difficulty of finding the optimal solution. With certain data modifications such as decreasing the demand in 
New Orleans for hurricane Katrina in the 51-scenario case study or decreasing the unmet demand penalty costs by $50 \%$, the shape of the objective function becomes somewhat flatter. This causes many solutions to become near optimal, (i.e. to have objective function values similar to the optimal solution). This increases the difficulty of finding the optimal solution, since only small changes in the objective function may result from large changes in the decision variables.

The following table lists the results obtained by running the experiments described in section 5.8 with a data set that either excludes the demand at New Orleans produced by hurricane Katrina, has reduced unmet demand penalty costs (by $50 \%$ ), or a combination of the two.

Table 6-15: Results for 51-scenarios with variations in demand for hurricane Katrina in New Orleans and unmet demand penalty costs obtained with LLSM and Lingo

\begin{tabular}{|c|c|c|c|c|c|c|c|}
\hline \multirow{2}{*}{ case } & \multirow{2}{*}{$\begin{array}{c}\text { Demand } \\
\text { reduction }\end{array}$} & \multirow{2}{*}{$\begin{array}{c}\text { Unmet } \\
\text { demand } \\
\text { penalty cost } \\
\text { reduction }\end{array}$} & \multicolumn{2}{|c|}{ Lagrangian L-shaped Method } & \multicolumn{2}{|c|}{ Lingo } \\
\cline { 4 - 9 } & & $\mathrm{z}$ & $\begin{array}{c}\text { time } \\
(\mathrm{s})\end{array}$ & iterations & $\begin{array}{c}\text { time } \\
(\mathrm{s})\end{array}$ & $\mathrm{Z}$ \\
\hline 1 & $\sqrt{ }$ & & $4.24243 \mathrm{E}+08$ & 49 & 48 & 1327 & $4.24202 \mathrm{E}+08$ \\
\hline 2 & $\sqrt{ }$ & & $4.25071 \mathrm{E}+08$ & 50 & 48 & 942 & $4.25189 \mathrm{E}+08$ \\
\hline 3 & $\sqrt{ }$ & & $4.26007 \mathrm{E}+08$ & 63 & 55 & 1390 & $4.26073 \mathrm{E}+08$ \\
\hline 4 & $\sqrt{ }$ & & $4.24183 \mathrm{E}+08$ & 77 & 63 & 1505 & $4.23933 \mathrm{E}+08$ \\
\hline 1 & & $\sqrt{ }$ & $3.40758 \mathrm{E}+08$ & 30 & 33 & 1303 & $3.40050 \mathrm{E}+08$ \\
\hline 2 & & $\sqrt{ }$ & $3.41032 \mathrm{E}+08$ & 26 & 30 & 763 & $3.40402 \mathrm{E}+08$ \\
\hline 3 & & $\sqrt{ }$ & $3.41274 \mathrm{E}+08$ & 29 & 32 & 1066 & $3.40579 \mathrm{E}+08$ \\
\hline 4 & & $\sqrt{ }$ & $3.40263 \mathrm{E}+08$ & 32 & 34 & 7498 & $3.39955 \mathrm{E}+08$ \\
\hline 1 & $\sqrt{ }$ & $\sqrt{ }$ & $2.24337 \mathrm{E}+08$ & 28 & 31 & $>86400$ & $2.234560+08$ \\
\hline 2 & $\sqrt{n}$ & $\sqrt{ }$ & $2.24326 \mathrm{E}+08$ & 28 & 31 & 9788 & $2.23709 \mathrm{E}+08$ \\
\hline 3 & $\sqrt{n}$ & $\sqrt{ }$ & $2.24269 \mathrm{E}+08$ & 24 & 28 & 609 & $2.23846 \mathrm{E}+08$ \\
\hline 4 & $\sqrt{ }$ & $\sqrt{ }$ & $2.23975 \mathrm{E}+08$ & 34 & 36 & $>86400$ & $2.23392 \mathrm{E}+08$ \\
\hline
\end{tabular}

Compared to Table 6-7, there is a large reduction in the objective values of the four cases with the demand reduction in New Orleans for hurricane Katrina (of 
approximately $2.3 \mathrm{E}+08$ ). The objective value for the cases with the $50 \%$ reduction in the unmet demand penalty costs were reduced by approximately $3.0 \mathrm{E}+08$. The cases that included both alterations (the $50 \%$ reduction of unmet demand penalty costs and the elimination of the demand in New Orleans due to hurricane Katrina) had objective values that decreased by approximately $4.2 \mathrm{E}+08$. These reductions in objective values show the influence in the solution caused by the particular data value changes.

One can notice from Table 6-15 that the running times of the experiments were consistent for the Lagrangian L-shaped method. For the commercial solver, on the other hand, the computational times increased as the surface of the feasible region became more "flat". The Lingo running times increased with the $50 \%$ reduction of the unmet demand penalty costs and even more so, with the complete reduction of the demand created by hurricane Katrina in New Orleans. The latter combination generated such a difficult mixed integer problem to solve, that it did not terminate in

over 24 hours for case 1 (no planes, no bottleneck) and case 4 (planes). Even though the objective values of the solutions obtained with the LLSM are within $1 \%$ of the objective values obtained with the commercial solver, the LLSM running times remained a fraction of the Lingo running times. The maximum LLSM running time was of 77 seconds, but was less than a minute in most cases.

\subsection{Tolerance}

This section includes the experiments conducted to determine the acceptable error threshold for the Lagrangian Relaxation and the Lagrangian L-shaped method. The example chosen for analysis was the 51-scenario case number 2 which includes the added helicopter links and the bottleneck in the links surrounding Atlanta, with 
unmet demand penalty costs equal to ten times the commodity purchase price. Table 6-16 shows the changes in objective value, number of iterations, computational time and error, as the tolerance changes on the outer linearization term $(\theta)$ approximating the second stage objective value $(H(y, r))$ for termination of the L-shaped method.

Figure 6-5 summarizes the major behavior graphically. From Table 6-16 and Figure 6-5 one can see that the error in the solution increases dramatically when the tolerance is larger than 0.001 . A tolerance level of 0.0001 seems a safe value to keep the error very small.

Table 6-16: Solution errors related to the tolerance on second-stage approximation

\begin{tabular}{|r|c|c|c|c|c|}
\hline tolerance & error & $\mathrm{z}$ & Percent error & iterations & time $(\mathrm{sec})$ \\
\hline 0.1 & $7.32901 \mathrm{E}+08$ & $6.65724 \mathrm{E}+08$ & 110 & 2 & 1 \\
\hline 0.01 & $6.65724 \mathrm{E}+08$ & $7.32901 \mathrm{E}+08$ & 91 & 2 & 1 \\
\hline 0.001 & $6.50976 \mathrm{E}+06$ & $6.60515 \mathrm{E}+08$ & 1 & 26 & 23 \\
\hline 0.0001 & $1.26573 \mathrm{E}+06$ & $6.58268 \mathrm{E}+08$ & 0 & 45 & 47 \\
\hline 0.00001 & $1.26573 \mathrm{E}+06$ & $6.58268 \mathrm{E}+08$ & 0 & 45 & 47 \\
\hline
\end{tabular}

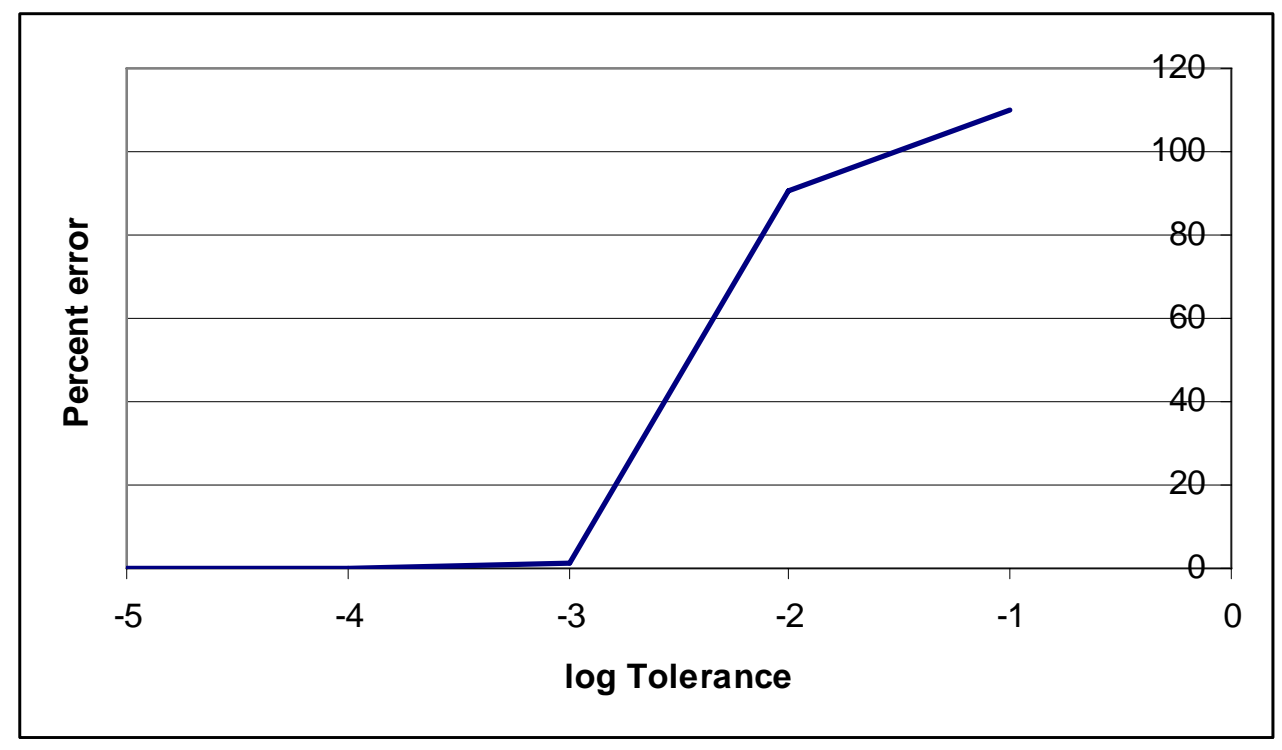

Figure 6-5: Graphical representation of the effect of tolerance 
Table 6-17: Tolerance error of the Lagrangian Relaxation

\begin{tabular}{|r|c|c|c|c|c|}
\hline tolerance & error & $\mathrm{Z}$ & Percent error & iterations & time $(\mathrm{sec})$ \\
\hline 0.1 & $5.40993 \mathrm{E}+07$ & $6.71912 \mathrm{E}+08$ & 8 & 13 & 10 \\
\hline 0.01 & $6.92979 \mathrm{E}+06$ & $6.60767 \mathrm{E}+08$ & 1 & 26 & 22 \\
\hline 0.001 & $1.26573 \mathrm{E}+06$ & $6.58268 \mathrm{E}+08$ & 0 & 45 & 47 \\
\hline 0.0001 & $1.26573 \mathrm{E}+06$ & $6.58268 \mathrm{E}+08$ & 0 & 46 & 49 \\
\hline 0.00001 & $1.26573 \mathrm{E}+06$ & $6.58268 \mathrm{E}+08$ & 0 & 46 & 49 \\
\hline
\end{tabular}

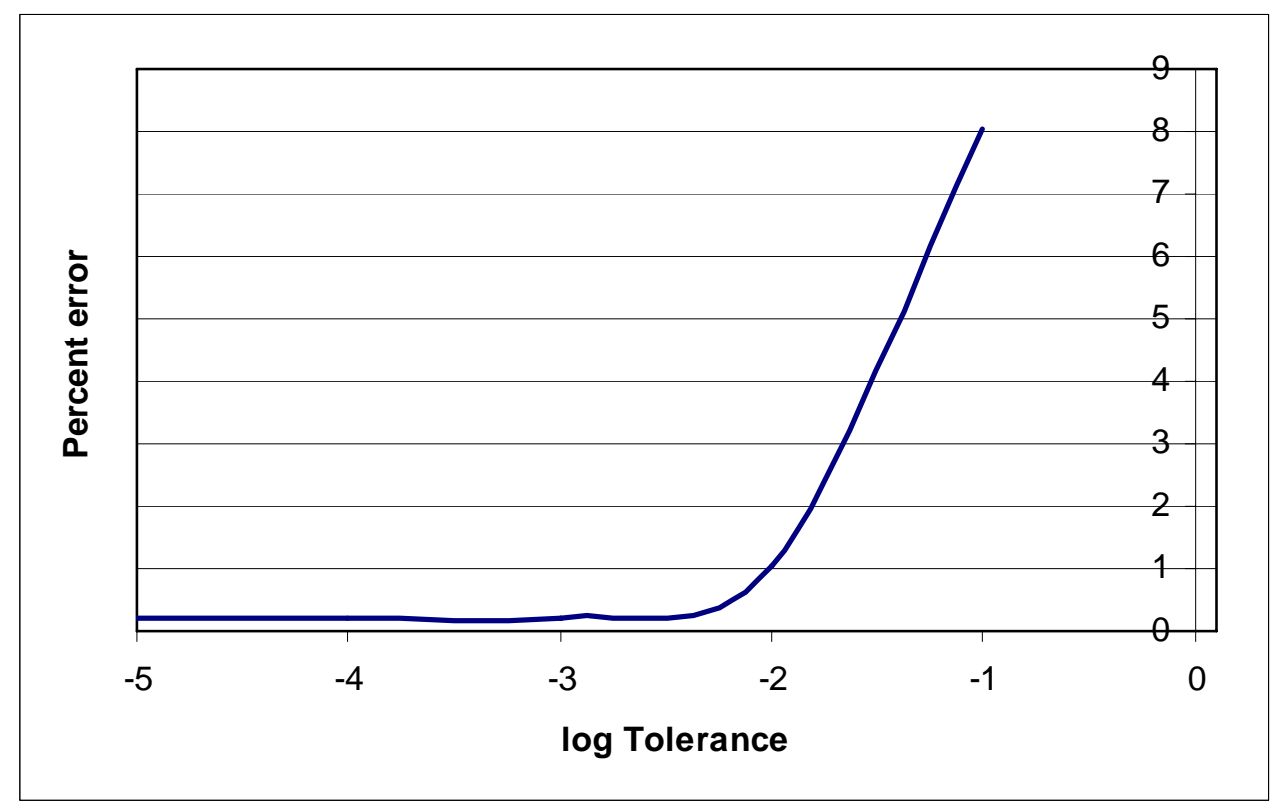

Figure 6-6: Graphical representation of the effect of tolerance in the Lagrangian relaxation

Table 6-17 shows similar data for changes in the tolerance level for the solution of the Lagrangian relaxation sub-problem. Figure 6-6 and Table 6-17 show the effect in the error term of relaxing the stopping criterion error threshold of the Lagrangian relaxation. The Lagrangian relaxation in this case, relaxes the facility capacity constraint (i.e. how much resource can be allocated to a facility based on its capacity). The error threshold chosen for the Lagrangian Relaxation was 0.001 since the magnitude of the error term remains constant for values smaller or equal to 0.001 . Figure 6-6 shows that for tolerance values greater than the one chosen, the error rate of 
the LLSM is strictly positive with a steeper slope shown for tolerance errors between 0.001 and 0.01 .

\subsection{Analysis of Results}

The experiments described in this chapter have highlighted five major findings with respect to the LLSM algorithm for solving the pre-positioning problem.

1) Although the algorithm is a heuristic, it consistently achieves solutions whose objective values are within $1 \%$ of optimum.

2) The solution times for the LLSM algorithm are at least an order of magnitude smaller than direct solution of the extensive form of the problem by Lingo, and are often two to three orders of magnitude smaller.

3) The extensive form direct solution time is very sensitive to constraints of link flows in the network, but the LLSM does not appear to be.

4) In the test cases analyzed here, there are multiple solutions that have nearly the same total cost. The solutions found by the LLSM and Lingo are often somewhat different (in number of facilities and acquisition of resources), but they have very similar objective values.

5) Tolerance parameters for both the Lagrangian relaxation and the L-shaped method are important to obtaining good solutions, but there are clear indications regarding how large those tolerances can be.

Based on these experimental findings, we conclude that the LLSM is a very effective way of solving pre-positioning problems. 


\section{CHAPTER 7. CONCLUSIONS}

\subsection{Closing remarks}

There is a need for facility location and resource allocation models that provide emergency response organizations with mitigation strategies for disaster threats to decrease the response time. For this purpose a mathematical formulation that provides a pre-positioning strategy for determining facility locations and resource allocation strategies was created. The primary goal of this model is to serve as a planning tool for disaster response. Even though the model can be solved in extensive form using a standard integer program solver when the data used is a small-scale case study, it was shown in section 6.2 that the computational capabilities of the latter can not tackle a large-scale version. For this reason, a heuristic solution that considers the embedded network structures of the stochastic mixed integer problem (SMIP) was devised by combining two methodologies: the L-shaped method and the Lagrangian relaxation.

The L-shaped method developed by Van Slyke and Wets (1969), consists of solving an approximation of a stochastic program by estimating the recourse function using an outer-linearization technique. Because the L-shaped method solves the SMIP using the estimated value of the recourse problem, the number of evaluations of the second stage problem decreases. The outer-linearization is performed by adding feasibility and optimality cuts to the relaxed mixed integer program version. Since the SMIP has complete recourse, only optimality cuts are needed.

The Lagrangian relaxation heuristic was added to decompose the first stage problem into a trivial facility location problem and a resource allocation linear program. To further improve the computational capabilities of the algorithm, the 
Lagrangian relaxation was used to relax the integrality constraints of the location variables. The result was a heuristic method referred to as the Lagrangian L-shaped method (LLSM).

Chapter 6 included the discussion of the results obtained for all the experiments. Analyses were performed using 21-scenario and 51-scenario case studies. Various experiments were performed to test the computational capabilities of the LLSM by changing the demand levels, by changing the number of scenarios, by adding helicopter links in the most damaged regions in the network, and by creating bottlenecks (i.e. decrease in capacity) on emanating links of a major city. These experiments showed the computational consistency of the method compared to a standard integer problem solver (i.e. Lingo). The running times for the LLSM remained between $0.05 \%$ and $10.0 \%$ of the Lingo running times. The difference in computational times between solvers did not compromise the quality of the solution. The solutions of the experimental cases solved using the LLSM were all within 1\% of the commercial solver objective values. The biggest differences observed were on the facility locations, but the total quantities of allocated supplies were similar.

It was observed that for this particular problem, the shape of the objective function changed with small fluctuations in the data set. When the shape of the objective function became flatter, the computational complexity of the problem increased because small changes in the objective value may have resulted from large changes in the decision variables. Two constants were altered to test the behavior of the solvers, the unmet demand penalty costs and the demand levels in the city of New Orleans during hurricane Katrina. While the computational times of the Lingo solver increased dramatically for certain 51-scenario case studies, the LLSM solved all the experiments in less than two minutes. 
The principal goal of this research was to provide an emergency response planning tool that developed large-scale resource allocation pre-positioning strategies for disaster threats. Based on the conducted experiments and its shown computational capabilities, we are confident that the LLSM is such a tool. Further experiments may be conducted to further test its capacity and limitations. The following section includes research proposed to test the full potential of the LLSM, steps toward a large-scale solution, and proposed modifications to improve its computational abilities.

\subsection{Next steps}

The immediate next step proposed is the creation of a large-scale problem to test the LLSM. This large-scale problem will include a larger level of detail in the network, with more cities (e.g. 100) and road segments. Instead of considering only trucks and helicopter links, railroad tracks will also be considered. Even though the latter has less flexibility (in terms of paths and travel time), it provides a large-capacity shipping mode. To determine a more accurate hurricane threat probability distribution, a more extensive hurricane history is required. Hurricane (or other alternative natural disaster) historical records will increase in number of events and in level of damage details.

It was seen that the solution of the LLSM improves with a larger array of facility types. So, the large-scale problem would include approximately 10 facility types of different dimensions. To provide a more complete resource pre-positioning plan, the solution should include more commodity types (e.g. 10 or more). These could include beds, blankets, lighting equipment, electricity generators, emergency vehicles, evacuation buses, etc. 
In the future, the model can be used for other emergency response problems. Not only can the LLSM be used for resource allocation for hurricane threats, but it could be used for preparations for natural disasters of another type or other planning problems altogether. One problem that could be studied is the preparation of shelters. The LLSM can determine the best location for shelters and the commodities needed for its operation. This type of planning is a little different than the case study solved, it may be performed with less anticipation or have less flexibility on the facility locations and capacities, but the LLSM can be modified to tackle this new problem. Other problems that could be solved with the LLSM are the planning of major suppliers that distribute merchandise nationwide, like a corporation distributing electric generators among its stores or a gas company supplying its vendors during hurricane season.

Another type of research that could be conducted is to modify elements of the LLSM. One could be to change the second stage minimum cost flow problems for multi-commodity flow problems, where the commodities would compete for the link capacities. Furthermore, in the case one needed to improve the computational efficiency of the algorithm, a parallel version can be considered. One can run the second stage problem evaluations in parallel time, since the network flow problems are independent per commodity and scenario. In the case of the multi-commodity flow problems, one can separate their evaluations by scenario only. Once all the evaluations are finished, the average value is calculated and the result is returned to obtain the objective function value for the current problem. 


\section{APPENDIX}

Appendix 1. Hurricane historical records from 1965 to 2004 provided by NOAA

\begin{tabular}{|c|c|c|c|c|c|}
\hline Year & Named storms & Hurricanes & Minor Hurricanes & Major hurricanes & $\mathrm{ACE}$ \\
\hline 1965 & 6 & 4 & 3 & 1 & 84 \\
\hline 1966 & 11 & 7 & 4 & 3 & 145 \\
\hline 1967 & 8 & 6 & 5 & 1 & 122 \\
\hline 1968 & 8 & 4 & 4 & 0 & 35 \\
\hline 1969 & 18 & 12 & 7 & 5 & 158 \\
\hline 1970 & 10 & 5 & 3 & 2 & 34 \\
\hline 1971 & 13 & 6 & 5 & 1 & 97 \\
\hline 1972 & 7 & 3 & 3 & 0 & 28 \\
\hline 1973 & 8 & 4 & 3 & 1 & 43 \\
\hline 1974 & 11 & 4 & 2 & 2 & 61 \\
\hline 1975 & 9 & 6 & 3 & 3 & 73 \\
\hline 1976 & 10 & 6 & 4 & 2 & 81 \\
\hline 1977 & 6 & 5 & 4 & 1 & 25 \\
\hline 1978 & 12 & 5 & 3 & 2 & 62 \\
\hline 1979 & 9 & 5 & 3 & 2 & 91 \\
\hline 1980 & 11 & 9 & 7 & 2 & 147 \\
\hline 1981 & 12 & 7 & 4 & 3 & 93 \\
\hline 1982 & 6 & 2 & 1 & 1 & 29 \\
\hline 1983 & 4 & 3 & 2 & 1 & 17 \\
\hline 1984 & 13 & 5 & 4 & 1 & 71 \\
\hline 1985 & 11 & 7 & 4 & 3 & 88 \\
\hline 1986 & 6 & 4 & 4 & 0 & 36 \\
\hline 1987 & 7 & 3 & 2 & 1 & 34 \\
\hline 1988 & 12 & 5 & 2 & 3 & 103 \\
\hline 1989 & 11 & 7 & 5 & 2 & 135 \\
\hline 1990 & 14 & 8 & 7 & 1 & 91 \\
\hline 1991 & 8 & 4 & 2 & 2 & 34 \\
\hline 1992 & 7 & 4 & 3 & 1 & 75 \\
\hline 1993 & 8 & 4 & 3 & 1 & 39 \\
\hline 1994 & 7 & 3 & 3 & 0 & 32 \\
\hline 1995 & 19 & 11 & 6 & 5 & 227 \\
\hline 1996 & 13 & 9 & 3 & 6 & 166 \\
\hline 1997 & 8 & 3 & 2 & 1 & 40 \\
\hline 1998 & 14 & 10 & 7 & 3 & 182 \\
\hline 1999 & 12 & 8 & 3 & 5 & 177 \\
\hline 2000 & 15 & 8 & 5 & 3 & 116 \\
\hline 2001 & 15 & 9 & 5 & 4 & 106 \\
\hline 2002 & 12 & 4 & 2 & 2 & 66 \\
\hline 2003 & 16 & 7 & 4 & 3 & 175 \\
\hline 2004 & 15 & 9 & 3 & 6 & 224 \\
\hline Average & 10.6 & 5.9 & 3.73 & 2.2 & 91 \\
\hline
\end{tabular}


Appendix 2. Hurricane incidence by region and category provided by NOAA

\begin{tabular}{|c|c|c|c|c|c|c|c|}
\hline \multirow{2}{*}{ AREA } & \multicolumn{6}{|c|}{ CATEGORY } & \multirow{2}{*}{$\begin{array}{c}\text { Major } \\
\text { Hurricanes }\end{array}$} \\
\hline & 1 & 2 & 3 & 4 & 5 & ALL & \\
\hline $\begin{array}{l}\text { U.S. Coastline } \\
\text { (Texas to Maine) }\end{array}$ & 113 & 74 & 76 & 18 & 3 & 284 & 97 \\
\hline Texas & 23 & 18 & 12 & 7 & 0 & 60 & 19 \\
\hline (North) & 12 & 7 & 3 & 4 & 0 & 26 & 7 \\
\hline (Central) & 7 & 5 & 2 & 2 & 0 & 16 & 4 \\
\hline (South) & 9 & 5 & 7 & 1 & 0 & 22 & 8 \\
\hline Louisiana & 18 & 14 & 15 & 4 & 1 & 52 & 20 \\
\hline Mississippi & 2 & 5 & 8 & 0 & 1 & 16 & 9 \\
\hline Alabama & 12 & 5 & 6 & 0 & 0 & 23 & 6 \\
\hline Florida & 44 & 33 & 29 & 6 & 2 & 114 & 37 \\
\hline (Northwest) & 27 & 16 & 12 & 0 & 0 & 55 & 12 \\
\hline (Northeast) & 13 & 8 & 1 & 0 & 0 & 22 & 1 \\
\hline (Southwest) & 16 & 8 & 7 & 4 & 1 & 36 & 12 \\
\hline (Southeast) & 13 & 13 & 11 & 3 & 1 & 41 & 15 \\
\hline Georgia & 12 & 5 & 2 & 1 & 0 & 20 & 3 \\
\hline South Carolina & 19 & 6 & 4 & 2 & 0 & 31 & 6 \\
\hline North Carolina & 22 & 13 & 11 & 1 & 0 & 46 & 13 \\
\hline Virginia & 9 & 2 & 1 & 0 & 0 & 12 & 1 \\
\hline Maryland & 1 & 1 & 0 & 0 & 0 & 2 & 0 \\
\hline Delaware & 2 & 0 & 0 & 0 & 0 & 2 & 0 \\
\hline New Jersey & 2 & 0 & 0 & 0 & 0 & 2 & 0 \\
\hline Pennsylvania & 1 & 0 & 0 & 0 & 0 & 1 & 0 \\
\hline New York & 6 & 1 & 5 & 0 & 0 & 12 & 5 \\
\hline Connecticut & 4 & 3 & 3 & 0 & 0 & 10 & 3 \\
\hline Rhode Island & 3 & 2 & 4 & 0 & 0 & 9 & 4 \\
\hline Massachusetts & 5 & 2 & 3 & 0 & 0 & 10 & 3 \\
\hline New Hampshire & 1 & 1 & 0 & 0 & 0 & 2 & 0 \\
\hline Maine & 5 & 1 & 0 & 0 & 0 & 6 & 0 \\
\hline
\end{tabular}




\section{REFERENCES}

Ahuja, Ravindra K., Thomas L. Magnanti, and James B. Orlin, (1993) Network Flows: Theory, Algorithms and Applications; Prentice Hall, USA, pp. 601-613, 674675.

Al-qurashi, Fahad (2004) New vision of emergency response planning. Process Safety Progress, Vol. 23, No. 1, pp. 56-61.

Atlantic Oceanographic and Meteorological Laboratory (AOML), "Frequently asked questions: hurricanes, typhoons, and tropical cyclones," Oceanic and Atmospheric Research (OAR) Facility of the National Oceanic and Atmospheric Administration (NOAA). http://www.aoml.noaa.gov/hrd/tcfaq/E11.html

Berman, Oded, Zvi Drezner, and George O. Wesolowsky, 2003, Locating service facilities whose reliability is distance dependent. Computers \& Operations Research, 30(11): 1683-1695.

Beven, Jack, 2005, “Tropical Cyclone Report Hurricane Dennis”, 4-13 July 2005, National Hurricane Center.

Birge, J. R. and F. Louveaux, 1997, Introduction to Stochastic Programming. Springer-Verlag, NY.

Bradley, Stephen P., Arnoldo C. Hax, and Thomas L. Magnanti, 1977, Applied Mathematical Programming; Addison-Wesley Publishing Co., Philippines: 512.

Chin, Hong-Chan, 1995, Optimal shunt capacitor allocation by fuzzy dynamic programming. Electric Power Systems Research, 35(2): 133-139.

Cornuejols, G., G. Nemhauser and L. A. Wolsey, 1990, The uncapacitated facility location problem. Discrete Location Theory, John Wiley, NY: 119-171.

El-Khattam, Walid, Y. G. Hegazy, and M. M. A. Salama, 2005, An integrated distributed generation optimization model for distribution system planning. IEEE Transactions on Power Systems, 20(2): 1158-1165. 
Fiedrich, F. F. Gehbauer, and U. Rickers, 2000, Optimized resource allocation for emergency response after earthquake disasters. Safety Science, 35(1): pp. 41-57.

Fisher, Marshall L., 1985, An applications oriented guide to Lagrangian relaxation, Interfaces, 15(2): 10-21.

Guha, Sudipto, Anna Moss, Joseph (Seffi) Naor, and Baruch Schieber, 1999, Efficient recovery from power outage. Proceedings of the 1999 31st Annual ACM Symposium on Theory of Computing - FCRC '99, May 1-May 4 1999, Atlanta, GA, USA: 574582.

Hsieh, Chung-Chi and Yi-Ting Chen, 2005a, Reliable and economic resource allocation in an unreliable flow network. Computers \& Operations Research, 32: 613628.

Hsieh, Chung-Chi and Yi-Ting Chen, 2005b, Resource allocation decisions under various demands and cost requirements in an unreliable flow network. Computers \& Operations Research, 32: 2771-2784.

Jia, Hongzhong, Fernando Ordoñez, and Maged M. Dessouky, 2007, Solution approaches for facility location of medical supplies for large scale emergencies, Computers and Industrial Engineering, 52(2): 257-276.

Johnstone, D. P., R. R. Hill and J. T. Moore, 2004, Mathematically modeling munitions prepositioning and movement. Mathematical and Computer Modelling, 39: $759-772$.

Lally, Brian J., 1991, Air Force Engineering \& Services, Military Engineer, 83 (540): 4-5.

Laporte, G. and F. V. Louveaux, 1993, "The integer L-shaped method for stochastic integer programs with complete recourse". Operations Research Letters, 13: 133-142.

Le Pera, Maurice E., Military Vehicles and Equipment, 2004, Tribology and Lubrication Technology, 60 (5): 29. 
Makram, Elham B., Dan Luke, Warren P. Adams, 1995, New technique for optimal size and location of capacitor banks in the presence of harmonics and distortion. Electric Power Systems Research, 34( 3): 149-156.

Mulvey, John M., Robert J. Vanderbei and Stavros A. Zenios, 1995, Robust optimization of large-scale systems. Operations Research, 43(2): 264-281.

National Oceanic and Atmospheric Administration website:

http://www.aoml.noaa.gov/hrd/tcfaq/E19.html

National Terror Alert Response Center website:

www.nationalterroralert.com/readyguide/safewater.htm

Neebe, Alan W., 1988, A procedure for locating emergency-service facilities for all possible response distances. The Journal of the Operational Research Society, 39(8): 743-748.

Ng, H. N. and M. M. A. Salama, 1995, Fuzzy optimal capacitor sizing and placement. Proceedings of the 1995 Canadian Conference on Electrical and Computer Engineering. Part 2 (of 2), Sep 5-8 1995, Montreal, Quebec, Canada; 2: 680-683.

Overseas Basing Commission, 2005, Report on Review of the Overseas Military Facility Structure of the United States. Arlington, VA.

http://www.lib.umich.edu/govdocs/pdf/obc.pdf

Parentela, Emelinda and Shashi Sathisan Nambisan, 2000, Emergency response (disaster management) Urban Planning and Development Applications of GIS: 181196.

Post, Buckley, Schuh \& Jernigan, Inc., 1990, Hurricane Hugo Assessment: Review of Hurricane Evacuation Studies Utilization and Information Dissemination, U. S. Army Corps of Engineers, Tallahassee, FL.

Post, Buckley, Schuh \& Jernigan, Inc., 1993, Hurricane Bonnie Assessment: Review of Hurricane Evacuation Studies Utilization and Information Dissemination, U. S. Army Corps of Engineers; Report No. 09-828.00, Tallahassee, FL. 
Post, Buckley, Schuh \& Jernigan, Inc., 1994, Hurricane Emily Assessment: Review of Hurricane Evacuation Studies Utilization and Information Dissemination, U. S. Army Corps of Engineers, Report No. 09-716.00, Tallahassee, FL.

Post, Buckley, Schuh \& Jernigan, Inc., 1999a, Hurricane Andrew Assessment: Review of Hurricane Evacuation Studies Utilization and Information Dissemination, U. S. Army Corps of Engineers, Report No. 09-683.00, Tallahassee, FL.

Post, Buckley, Schuh \& Jernigan, Inc., 1999b, Hurricane Georges Assessment: Review of Hurricane Evacuation Studies Utilization and Information Dissemination, U. S. Army Corps of Engineers, Report No. 09-831.00, Tallahassee, FL.

Post, Buckley, Schuh \& Jernigan, Inc., 2000, Hurricane Floyd Assessment: Review of Hurricane Evacuation Studies Utilization and Information Dissemination, U. S. Army Corps of Engineers, Report No. 090857.00, Tallahassee, FL.

Post, Buckley, Schuh \& Jernigan, Inc., 2003, Hurricane Lili Post Storm Assessment with effects from Tropical Storm Isidore: Review of Hurricane Evacuation Studies Utilization and Dissemination, U. S. Army Corps of Engineers, Report No.

091223.00, Tallahassee, FL.

Post, Buckley, Schuh \& Jernigan, Inc., 2005, Hurricane Isabel Assessment: Review of Hurricane Evacuation Study Products and Other Aspects of the National Hurricane Mitigation and Preparedness Program (NHMPP) in the Context of the Hurricane Isabel Response, U. S. Army Corps of Engineers, Report No. 091247.00, Tallahassee, FL.

Saccommano, F. F. and B. Allen, 1988, Locating emergency response capability for dangerous goods incidents on a road network, Transportation Research Record, 1193: $1-9$.

Sathe, Aamod and Elise Miller-Hooks, 2005, Optimizing location and relocation of response units in guarding critical facilities, Transportation Research Record, 1923: 127-136.

Savage, R. P., J. Baker, J. H. Golden, A. Kareem, and B. R. Manning, Hurricane Alicia: Galveston and Houston, Texas, August 17-18, 1983, Report No. CETS-CND028, National Academy Press, Washington, DC, 1984. 
Sherali, Hanif D., Jitamitra Desai, and Theodore S. Glickman, 2004, Allocating emergency response resources to minimize risk with equity considerations. American Journal of Mathematical and Management Sciences, 24(3-4): 367-410.

Snyder, Lawrence V. and Mark S. Daskin, 2005, Reliability for facility location: the expected failure cost case. Transportation Science, 39(3): 400-416.

Syam, Siddhartha (1997) A model for the capacitated p-facility location problem in global environments. Computers \& Operations Research, 24(11): 1005-1016.

Toregas, Constantine, Charles ReVelle and Lawrence Bergman, 1970, The location of emergency service facilities. Operations Research, 19: 1363-1373.

Tsai, Yichang (James), Zhaohua Wang, and Chien-Tai Yang, 2002, A prototype realtime GPS/GIS-based emergency response system for locating and dispatching moving patrol vehicles with the beat-based shortest distance search, Traffic and Transportation Studies Proceedings of ICTTS, 2: 1361-1368.

U. S. Army Corps of Engineers Charleston District, 1998, Hurricane Bertha and Fran Assessment: Review of the use and value of hurricane evacuation studies in the Hurricane Bertha evacuation July 9-12, 1996 and, in the Hurricane Fran evacuation September 3-6, 1996; Georgia, South Carolina, North Carolina and Virginia.

U. S. Army Corps of Engineers Mobile District, 1970, Report on Hurricane Camille 14-22 August 1969.

U. S. Army Corps of Engineers Philadelphia District, 1996, Hurricane Opal Assessment: Review of the use and value of hurricane evacuation study products in the Hurricane Opal evacuation, Alabama and Florida, October 3-4, 1995.

U. S. Department of Commerce, National Oceanic and Atmospheric Administration, and National Weather Service, 1997a, Service Assessment Hurricane Bertha: July 514, 1996.

U. S. Department of Commerce, National Oceanic and Atmospheric Administration, and National Weather Service, 1997b, Service Assessment Hurricane Fran: August 28-September 8, 1996. 
U. S. Department of Commerce, National Oceanic and Atmospheric Administration, and National Weather Service, 2006, Service Assessment Hurricane Katrina: August 23-31, 2005; Silver Spring, Maryland.

Van Slyke, R. M. and Roger Wets, 1969, L-shaped linear programs with applications to optimal control and stochastic programming. Journal on Applied Mathematics, 17(4): 638-663.

Weintraub, A., J. Aboud, C. Fernandez, G. Laporte, and E. Ramirez, 1999, An emergency vehicle dispatching system for an electric utility in Chile. Journal of the Operational Research Society, 50: 690-696. 